\title{
Functional studies on the transmembrane protein encoded by the TM20 gene in maize
}

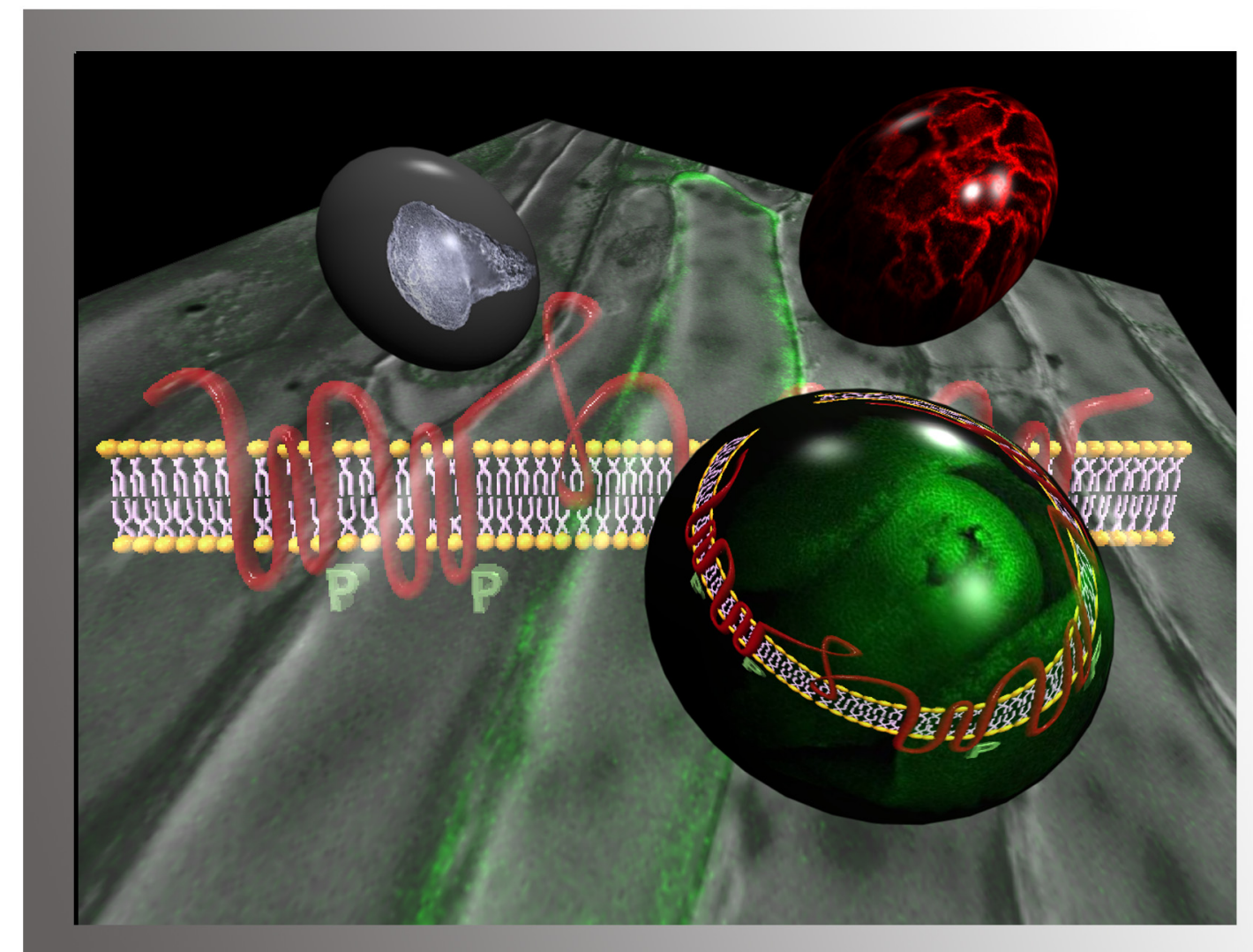

Göttingen-Barcelona 2002 


\title{
Functional studies on the transmembrane protein encoded by the TM20 gene in maize
}

\author{
Dissertation \\ zur Erlangung des Doktorgrades \\ der Mathematisch-Naturwissenschaftlichen Fakultäten \\ der Georg-August-Universität zu Göttingen
}

\author{
vorgelegt von \\ Torben Jahrmann \\ aus Göttingen
}

Göttingen 2002 
D 7

Referent:

Korreferent:

Tag der mündlichen Prüfung:
Prof. Dr. D. Gradmann

Prof. Dr. C. Gatz 


\title{
Functional studies on the transmembrane protein encoded by the TM20 gene in maize
}

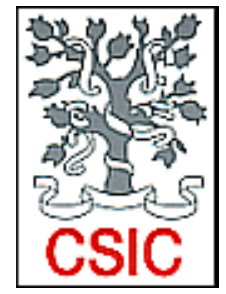 \\ Institut de Biología Molecular Barcelona (IBMB) \\ Centre d'Investigació I Desenvolupament (CID) \\ Consell Superior d'Investigacions Científiques (CSIC)
}

Barcelona 2002 



\section{To Mónica and Kaira}




\section{In memory of Virginia}


Contents 


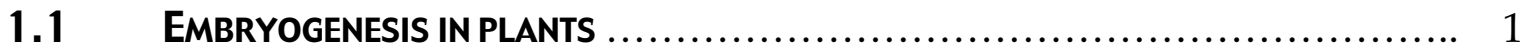

1.1.1 Embryogenesis in Arabidopsis thaliana ................................. 2

1.1.2 Embryogenesis in Zea mays ....................................... 3

1.1.3 Genetical approach to study embryogenesis of plants .................... 5

1.1.4 Molecular approach to study embryogenesis of plants ................... 7

1.2 PATTERN FORMATION DURING PLANT EMBRYOGENESIS .......................... 8

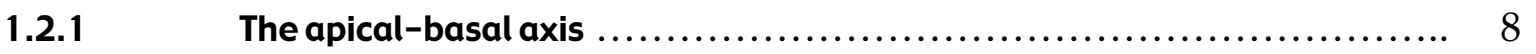

1.2.2 Establishment of the radial pattern .................................... 10

1.3 THE ROLE OF AUXIN AND POLAR AUXIN TRANSPORT IN EMBRYOGENIC AND

POST-EMBRYOGENIC PATTERN FORMATION ............................... 12

1.3.1 Polar auxin transport .............................................. 14

1.4 THE MAIZE DEFECTIVE KERNEL MUTANT LACHRIMA FROM ZEA MAYS .............. 18

1.5 THE AIM OF THE PRESENT STUDY ............................................... 21

2 Material and Methods............................................. 23

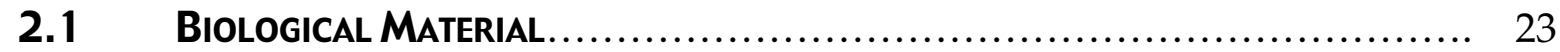

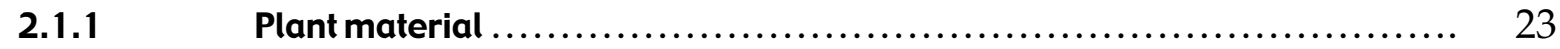

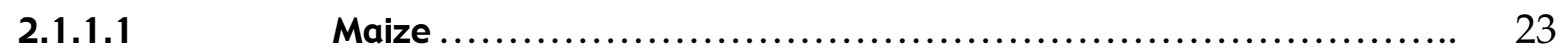

2.1.1.2 Arabidopsis..................................................... 23

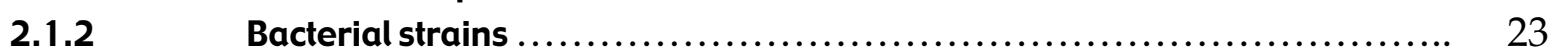

2.1.2.1 Preparation of competent E.colicells ............................. 24

2.1.2.2 Transformation of competent E.colicells .......................... 24

2.1.3 Vectors and Oligonucleotides ...................................... 24

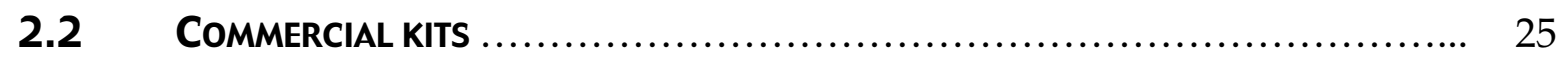

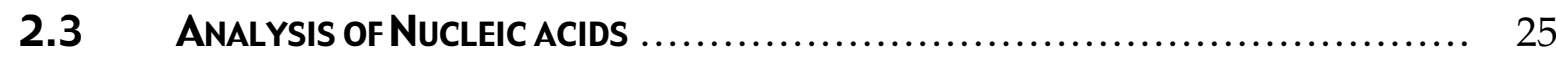

2.3.1 Extraction and purification of DNA from E.coli (Minipreps and Midipreps) ... 25

2.3.2 Extraction and purification of RNA from plants ........................... 26

2.3.2.1 Phenol/Chloroform extraction of plant RNA ........................ 27

2.3.2.2 RNA extraction with the RNA easy plant kit ......................... 27

2.3.3 DNA digestion with restriction enzymes and ligation reactions ............. 27

2.3.4 Dephosphorylation reaction with Alkaline Phosphatase (AP) .............. 28

2.3.5 Generation of blunt ends by Klenow fragment reaction .................... 29

2.3.6 Gel electrophoresis .............................................. 29

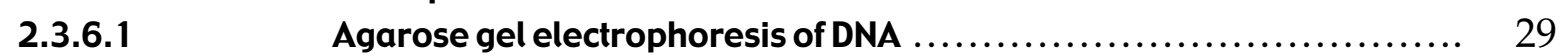

2.3.6.2 RNA Agarose gel electrophoresis under denaturating conditions ...... 29

2.3.6.3 Purification of DNA bands from agarose gels ...................... 30

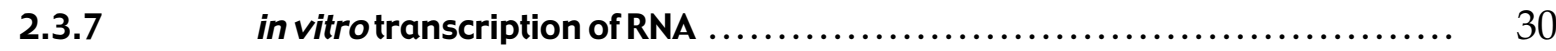

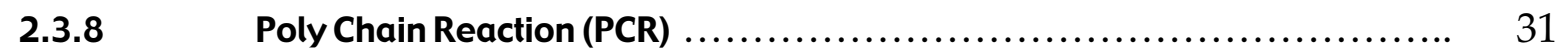

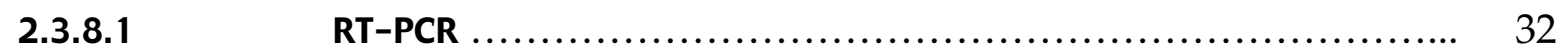




\section{Contents}

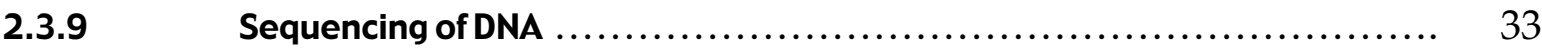

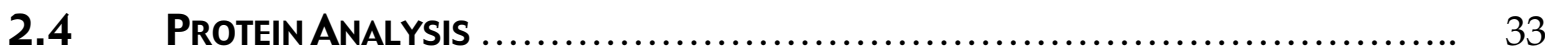

2.4.1 Protein Extractions from plants .................................... 33

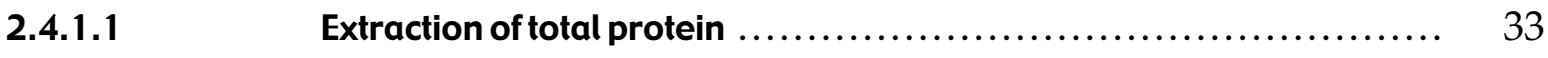

2.4.1.2 Extraction and separation of membrane fractions by differential

2.4.1.3 Protein precipitation with TCA and acetone .......................... 34

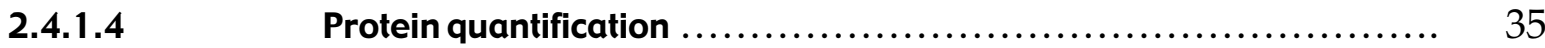

2.4.2 Expression and purification of recombinant protein in E.coliusing the pET system .................................................... 35

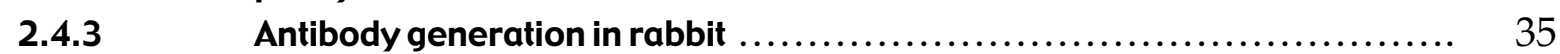

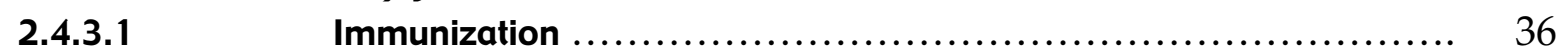

2.4.3.2 Antibody preparation, storage and purification $\ldots \ldots \ldots \ldots \ldots \ldots \ldots \ldots \ldots \ldots$

2.4.4 Immunodetection and immunohistochemistry ....................... 37

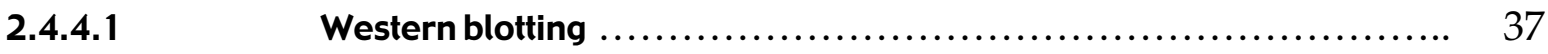

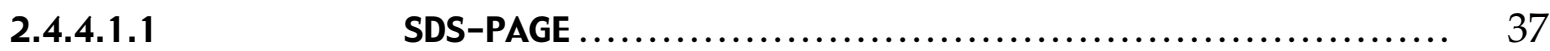

2.4.4.1.2 Electroblotting $\quad$. $\quad$.

2.4.4.1.3 Immunodetection ........................................ 38

2.4.4.1.4 Gel coomassie blue staining .............................. 39

2.4.4.2 Immunolocalization in plant tissue ............................. 39

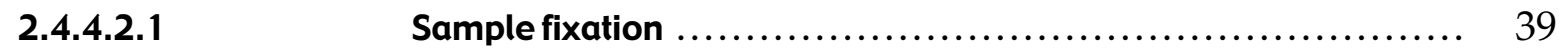

2.4.4.2.2 Embedding in paraffin ..................................... 40

2.4.4.2.3 Sample sectioning ........................................... 41

2.4.4.2.4 Immunodetection ......................................... 41

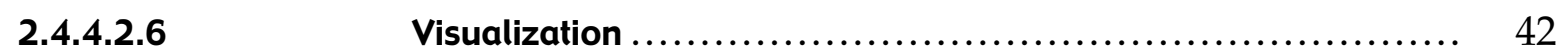

2.4.4.2.6.1 Light fluorescence microscopy ........................ 42

2.4.4.2.6.2 Confocal laser scanning microscopy ..................... 43

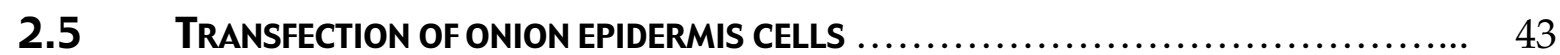

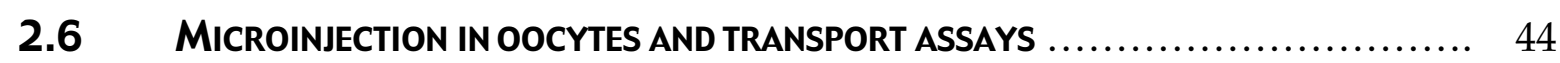

2.6.1 Isolation of oocytes from Xenopus laevis ............................... 44

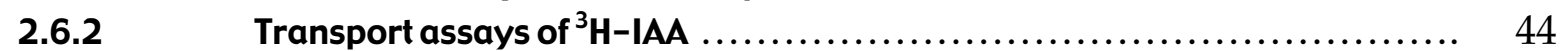

2.6.3 Diffusion test for IAA in oocytes ...................................... 45

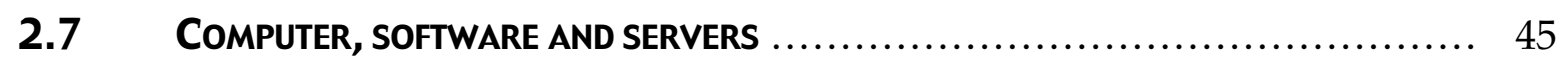

3 Results............................................................. 47

3.1 SEARCH FOR TM20 IN SEVERAL PLANTS.......................................... 47

3.1.1 TM 20 in Oryza sativa..................................................... 47

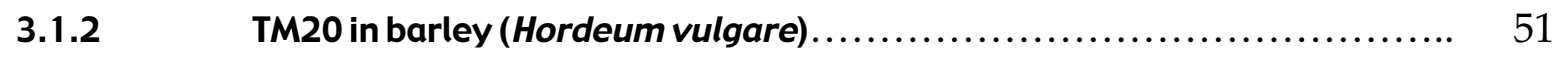

3.1.3 TM20 in Arabidopsis thaliana.......................................... 52

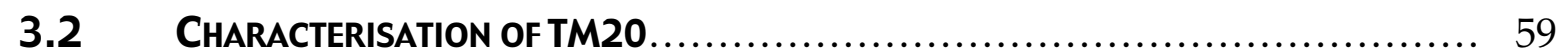

3.2.1 Generating antibodies ............................................... 59

3.2.1.1 Expression of the loop region in pET 28a.............................. 60

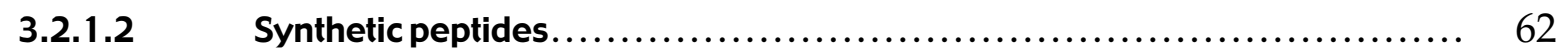

3.2.2 Characterisation of the antibodies...................................... 63

3.2.2.1 Antibodies against the recombinant LoopTM20......................... 63

3.2.2.2 Antibodies against the synthetic peptides................................ 64 
Detection of TM20 in maize embryos

TM20 detection in microsomes of maize embryos at different developmental stages SDS-PAGE and western analysis of 20DAP maize embryo microsomes. Immunolocalization on 15DAP maize embryo sections................... 75 Whole mount immunolocalization on 10DAP maize embryos.............. 76 Immunolocalization on lachrima mutants............................. 78 Detection of an ${ }^{+} \mathrm{H}$-ATPase in 10DAP maize embryos........................ 79 Detection of TM20 in Black mexican sweet (BMS) cultured cells............ 80 The presence of IAA did not modify the detection pattern of TM20 in 20DAP embryos.

\subsection{EXPRESSION OF A TM20:GFP FUSION PROTEIN IN TRANSFECTED ONION} EPIDERMIS CELLS.

MICROINJECTION IN OOCYTES OF XENOPUS LAEVIS.......................... 88

Microinjection of cRNA of TM2O in oocytes and immunocytochemistry on oocyte sections.

Addition of an auxin transport inhibitor (NPA) increases accumulation of $3 \mathrm{H}-\mathrm{IAA}$ in TM2O injected oocytes.

3.5.7 No difference in IAA accumulation is observed between control oocytes and oocytes injected with AtTM4.

\section{Discussion}

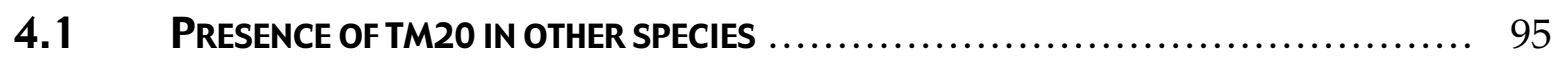

4.2 LOCALIZATION OF TM2O IN THE MAIZE EMBRYO ................................ 99

4.3 THE CAPACITY OF TM20 TO TRANSPORT IAA ACROSS MEMBRANES .................. 102

4.4 IS TM2O PART OF THE AUXIN TRANSPORT SYSTEM ? .......................... 105 



\section{Abstract}




\section{Abstract}

In the course of the analysis of lachrima, a recessive, defective kernel, embryolethal mutation in Zea mays that blocks embryo and endosperm development at transition stage, a gene coding for a new class of transmembrane proteins was isolated. The predicted protein contains twenty hydrophobic segments that can be grouped in five repeats formed by four segments that fulfil the criteria for membrane spanning domains, and for this reason the gene has been named TM20. The phenotype of lachrima displays a high similarity to wild-type embryos grown in the presence of an excess of auxin. This, together with the structural characteristics of a protein with transporter function gives rise to the hypothesis that TM20 is related to the mechanism of auxin-transport. In this study the localization of TM20 in the maize embryo and at the subcellular level was investigated and its possible involvement in auxin transport processes by expression of TM20 in heterologous systems. With the aim of localizing TM20 by immunological methods various antibodies were raised against specific zones of the protein and the association of TM20 to membranes demonstrated by western and dot blot analysis. TM20 was detected in maize embryos of different developmental stages in the leaf primordia, the shoot apical meristem, the radicle and in cell layers surrounding the provascular tissue. At the subcellular level TM20 is located mainly at the apical part of the cells in a polar manner. A similar polar localization at the basal or the apical part of the cell was described also for the putative auxin carriers from Arabidopsis PIN1, PIN2 and AUX1. Furthermore, the definition of the polarized pattern of TM20 seems to be dependent on the developmental stage of the cells, since no polarisation of TM20 was found in young embryos (10DAP) or in the undifferentiated cells of the shoot apical meristem. With the purpose of testing whether TM20 transports IAA the protein was expressed in oocytes of Xenopus laevis. The transport assays showed that radiolabeled IAA entered the cells by diffusion depending on the $\mathrm{pH}$ of the medium. Moreover, oocytes expressing TM20 accumulate up to 50\% less IAA after 60 min of transport than the corresponding controls, pointing to an IAA-efflux activity of TM20. The addition of the known auxin transport inhibitor N-1-Naphthylphtalamic acid (NPA) 


\begin{abstract}
reduced the accumulation of IAA in TM20 expressing oocytes to levels of the control oocytes, indicating the specifity of the observed transport effect. An extensive search in the databases lead to possible homologues of TM20 in Oryza sativa, Arabidopsis and Hordeum vulgare. The in silico analysis of these homologues and the cloning of a TM20-like cDNA from Arabidopsis by RT-PCR suggests the presence of TM20 in more plant species. The results of the immunohistochemistry and the observed transport ability of TM20 suggests the involvement of TM20 in the formation of an auxin gradient in the plant.
\end{abstract}




\section{List of abbreviations}

\begin{tabular}{|c|c|}
\hline aa & amino acid \\
\hline$A c$ & Activator \\
\hline ATP & adenosine triphosphate \\
\hline $\mathrm{AP}$ & alkaline phosphatase \\
\hline BMS & Black mexican sweet \\
\hline BrET & ethidium bromide \\
\hline BSA & bovine serum albumin \\
\hline $\mathrm{bp}$ & base pairs \\
\hline${ }^{\circ} \mathrm{C}$ & celsius \\
\hline cDNA & complementary DNA \\
\hline CFA & complete Freunds adjuvant \\
\hline $\mathrm{CH}_{3} \mathrm{Cl}$ & chloroform \\
\hline $\mathrm{Ci}$ & curie \\
\hline cRNA & complementary RNA \\
\hline dek & defective kernel \\
\hline dNTP & deoxyribonucleotide phosphate \\
\hline DNA & deoxyribonucleic acid \\
\hline DNase & deoxyribonuclease \\
\hline DTT & dithiothreitol \\
\hline ECL & enhanced chemiluminescence \\
\hline E.coli & Escherichia coli \\
\hline EDTA & ethylenediamine tetraacetic acid \\
\hline ER & endoplasmic reticulum \\
\hline EST & expressed sequence tag \\
\hline $\mathrm{EtOH}$ & ethanol \\
\hline GL & ground leaves \\
\hline $\mathrm{g}$ & gram \\
\hline GFP & green fluorescence protein \\
\hline $\mathrm{h}$ & hour \\
\hline${ }^{3} \mathrm{H}$ & tritium \\
\hline $\mathrm{HCl}$ & hydrochloric acid \\
\hline HRP & Horse raddish peroxidase \\
\hline IAA & Indoleacetic acid \\
\hline $\operatorname{IgG}$ & immunoglobulin $\mathrm{G}$ \\
\hline IFA & incomplete Freunds adjuvant \\
\hline IPTG & isopropyl $ß$-D-thiogalactopyranoside \\
\hline IsoAA & isoamyl alcohol \\
\hline $\mathrm{kb}$ & kilobase \\
\hline KDEL & signal for endoplasmic reticulum retaintion \\
\hline $\mathrm{L}$ & leaves \\
\hline LB & Luria Bertani broth \\
\hline M & molar (moles per litre) \\
\hline MBS & Modified Barth Solution \\
\hline $\mathrm{MeOH}$ & methanol \\
\hline$\mu g$ & microgram \\
\hline$\mu 1$ & microlitre \\
\hline
\end{tabular}




\begin{tabular}{|c|c|}
\hline$\mu \mathrm{M}$ & micromolar \\
\hline $\mathrm{mg}$ & milligram \\
\hline $\mathrm{mM}$ & millimolar \\
\hline $\min$ & minute \\
\hline $\mathrm{ml}$ & millilitre \\
\hline mRNA & messenger RNA \\
\hline MW & molecular weight \\
\hline ng & nanogram \\
\hline $\mathrm{nl}$ & nanolitre \\
\hline $\mathrm{NaCl}$ & sodium chloride \\
\hline $\mathrm{NaN}_{3}$ & sodium azide \\
\hline $\mathrm{NPA}^{\circ}$ & N-1-Naphthylphtalamic acid \\
\hline OD & optical density \\
\hline ON & over night \\
\hline PAT & polar auxin transport \\
\hline pCK GFP & GFP cloning vector \\
\hline PCR & polymerase chain reaction \\
\hline pKAR6 & $\begin{array}{l}\text { GFP vector expressing GFP with a targeting peptide of a } \\
\text { chitimase and a KDEL sequence }\end{array}$ \\
\hline Pl & plantlets \\
\hline pmol & picomolar \\
\hline PMSF & phenylmethylsulfonyl fluoride \\
\hline PVDF & polyvinylidene fluoride \\
\hline $\mathrm{R}$ & root of plantlets \\
\hline RNA & ribonucleic acid \\
\hline RNase & ribonuclease \\
\hline RNAsin & inhibitor of ribonuclease activity \\
\hline rpm & revolution per minute \\
\hline RT & room temperature \\
\hline RT-PCR & reverse transcriptase poly chain reaction \\
\hline SDS & sodium dodecyl sulfate \\
\hline SDS-PAGE & SDS-polyacrylamide gel electrophoresis \\
\hline sec & second \\
\hline Sl & siliqua \\
\hline St & stem \\
\hline St2 & basal part of the stem \\
\hline St3 & middle part of the stem \\
\hline Taq & Thermus aquaticus \\
\hline TCA & trichloroacetic acid \\
\hline TIBA & 2,3,5-triiodobenzoic acid \\
\hline TM20 & transmembrane 20 \\
\hline TMx2 & protein loading buffer \\
\hline $\mathrm{U}$ & unit \\
\hline UV & ultraviolet \\
\hline Vol & volume \\
\hline$w / v$ & weight : volume ratio \\
\hline
\end{tabular}




\section{Introduction}




\section{Introduction}

Embryogenesis in eukaryotes is a critical process, as the basis of the development of an adult individual (Harada 1999). Nevertheless, several features exist that distinguish plant embryogenesis from the well-studied embryonic development in animals.

An important difference between animal and plant cells is that plants have cell walls. Because of the immobility of the plant cells no cell migration occurs during embryogenesis and the changes in morphogenesis are driven by orientated cell division and directional cell expansion (Lloyd 1991; Meyerowitz 1997).

\subsection{Embryogenesis in plants}

During embryogenesis in higher plants, the meristems, the shoot-root body pattern of the plant and the primary plant cell types are specified (Goldberg et al. 1989; Goldberg et al. 1994). The questions, how the embryo acquires its threedimensional shape with specialized organs and tissues, and what gene networks control and regulate the multiple fine-regulated processes of embryonic development, are still unanswered. It is well known that development of endosperm and embryo requires distinct genetic programmes and is essential for the development of the new sporophytic generation (Goldberg, Barker et al. 1989; Meinke 1991; Goldberg, de Paiva et al. 1994).

To study plant embryogenesis a multidisciplinary approach was used. The structural changes of the seed, which contains the embryo and the endosperm were described using microscopy techniques (Johansen 1950; Wardlaw 1955). As a genetical approach mutants with obvious deficiencies in the early development were generated. In this context, the techniques of molecular biology have become important tools for the analysis of these mutants and the responsible genes, providing a better comprehension of embryogenesis (Meinke 1991; Meinke 1995). 


\subsubsection{Embryogenesis in Arabidopsis thaliana}

The Arabidopsis embryo development can be divided in a morphogenetic stage, maturation stage and dormancy (Fig. 1.1). The morphogenetic stage is subdivided in the post-fertilization - proembryo stage and the globular - heart transition stage. During post-fertilization-proembryo stage the polarized zygote divides asymmetrically into a small, protoplasm rich cell and a large, vacuolated cell. This division is the first step of the proembryo formation with a suspensor and embryo proper. The embryo proper will form the major part of the mature embryo, whereas one cell of the suspensor, the uppermost cell called the hypophysis, will participate in the formation of the radicule region of the embryo axis (Mansfield and Briarty 1990; Dolan et al. 1993). The rest of the suspensor has the task of projecting the embryo proper into the maternal tissue and of providing the nutrients and growth factors (Yeung and Meinke 1993; Souter and Lindsey 2000). But besides the mechanical task, the suspensor is known to be actively involved in regulating the development of the embryo proper during early embryogenesis (Yeung 1980; Meinke 1991).

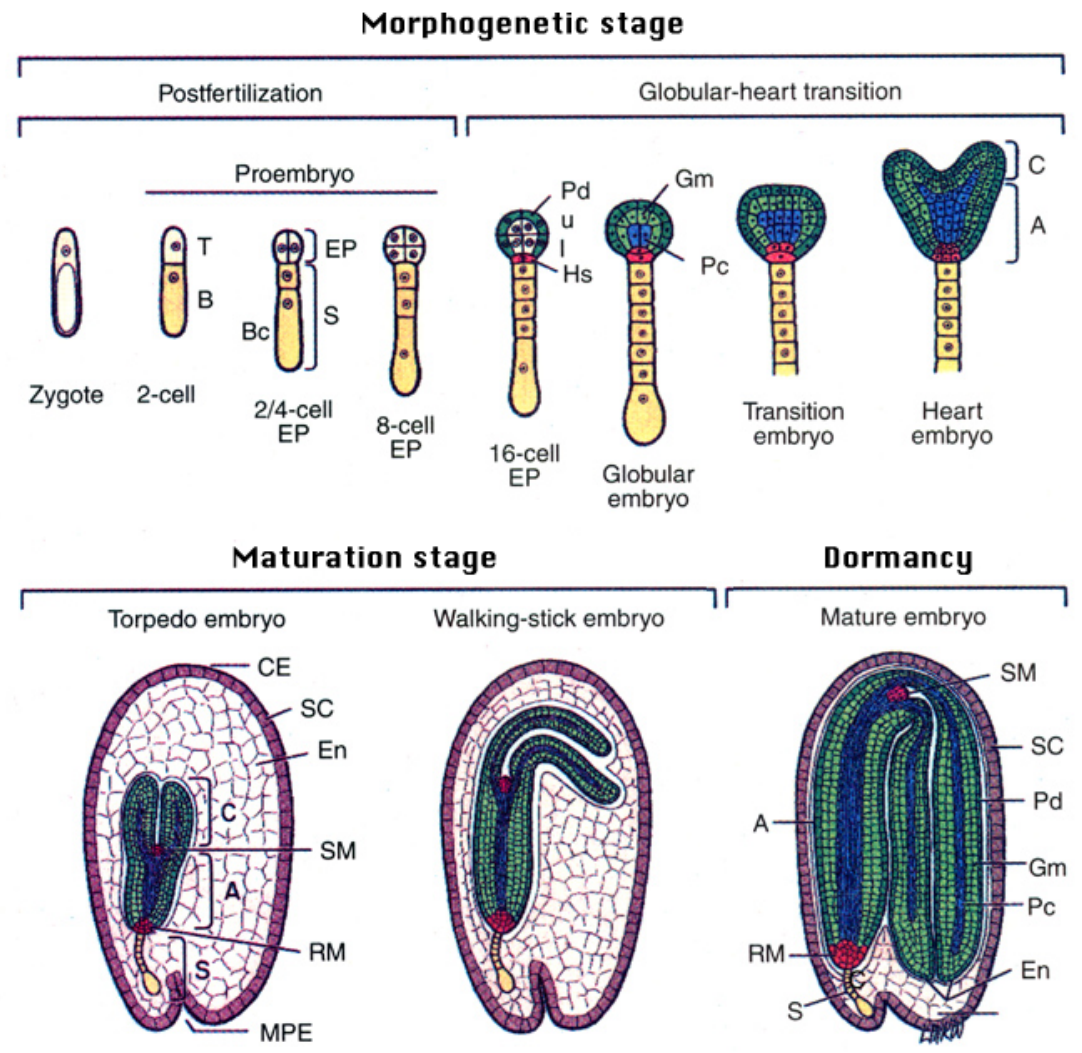

Figure 1.1: A generalized overview of plant embryogenesis. Schematic representations of embryonic stages are based on light microscopy studies of Arabidopsis and Capsella embryo development. Torpedo and walking-stick refer to specific stages of embryogenesis in Arabidopsis and Clapsella. Abbreviations: T, terminal cell; $\mathbf{B}$, basal call; EP, embryo proper; $\mathbf{S}$, suspensor; Bc, suspensor basal cell; Pd, protoderm; u, upper tier; I, lower tier; Hs, hypophysis; Pc, procambium; $\mathbf{G} \mathbf{m}$, ground meristem; C, cotyledon; $\mathbf{A}$, axis; M P E , micropylar end; C E, chalazal end; SC, sedd coat; En, endosperm; SM, shoot meristem; and $\mathbf{R M}$, root meristem. Adapted from Goldberg et al (1994) with modifications. 
In the globular - heart transition stage the embryonic organs and tissue-types are differentiated and the morphology of the embryo changes. By periclinal cell divisions of the embryo proper the protoderm is constituted. Subsequently the cotyledons emerge by differential cell division at the lateral margins of the globular shaped embryo. As a consequence of the growth of the cotyledons the embryo shifts from a radial symmetry to a bilateral symmetry. This symmetry change is characteristic of the heart stage (West and Harada 1993; Goldberg, de Paiva et al. 1994). By this time the body organization and the cellular layers of the mature embryo (and post embryonic plant) have been established.

The maturation stage is characterized by organ expansion and the preparation of the seed for dormancy. The pattern formation program is switched to a program controlling the accumulation of storage proteins for post-embryonic development. In the case of Arabidopsis the main part of the storage products are accumulated in the cotyledons. During seed development the endosperm is almost totally assimilated by the growing embryo. By the end of the maturation stage the embryo has reached his maximum size, the surrounding seed layers are dehydrated and the metabolic activities have decreased. The period of dormancy begins (Goldberg, de Paiva et al. 1994).

\subsubsection{Embryogenesis in Zea mays}

The embryogenesis of Zea may can also be divided into morphogenetic stage, maturation stage and dormancy (Fig. 1.2). During the morphogenetic stage the polarisation of the zygote is established and the proembryo is formed. The proembryo is composed of the embryo proper and the suspensor. The following part of the morphogenetic stage is characterized by a high morphological activity and the differentiation of the embryo axis and the scutellum and ends in the formation of the first leaf primordia. In the following stage of maturation the growth of the elaborated structures continues, storage proteins are accumulated and the embryo is prepared for dormancy (Randolph 1936; Raghavan 1976; Clark 1996).

24-48 hours after fertilization the first division of the zygote takes place giving a small apical daughter cell with dense protoplasm and a bigger and vacuolated basal 
daughter cell. The subsequent irregular anticlinal and periclinal cell divisions occur mainly in the apical part and form a club shaped proembryo. The proembryo now has radial symmetry and is composed of the embryo proper, from the small apical daughter cell and the suspensor, originating from the elongated basal cell. At the end of the proembryo stage the protoderm is differentiated (Fig. 1.2) (Randolph 1936; van Lammeren 1986). The late morphogenetic stage is characterized by a change in embryo symmetry. The differentiation of the embryo axis and the scutellum causes a switch from a radial symmetry to a bilateral symmetry. The development of the apical meristem is histologically visible in the late transition stage as a group of small, dense cells at the adaxial side of the transition stage embryo (Fig. 1.2) (Randolph 1936). During the coleoptilar stage the coleoptilar ring forms around the apical meristem and the basal part of the embryo proper differentiates into the root meristem. The formation of the first leaf primordia occurs just beneath the shoot apical meristem, which characterizes the L1-stage.

\section{Morphpgenetic stage}

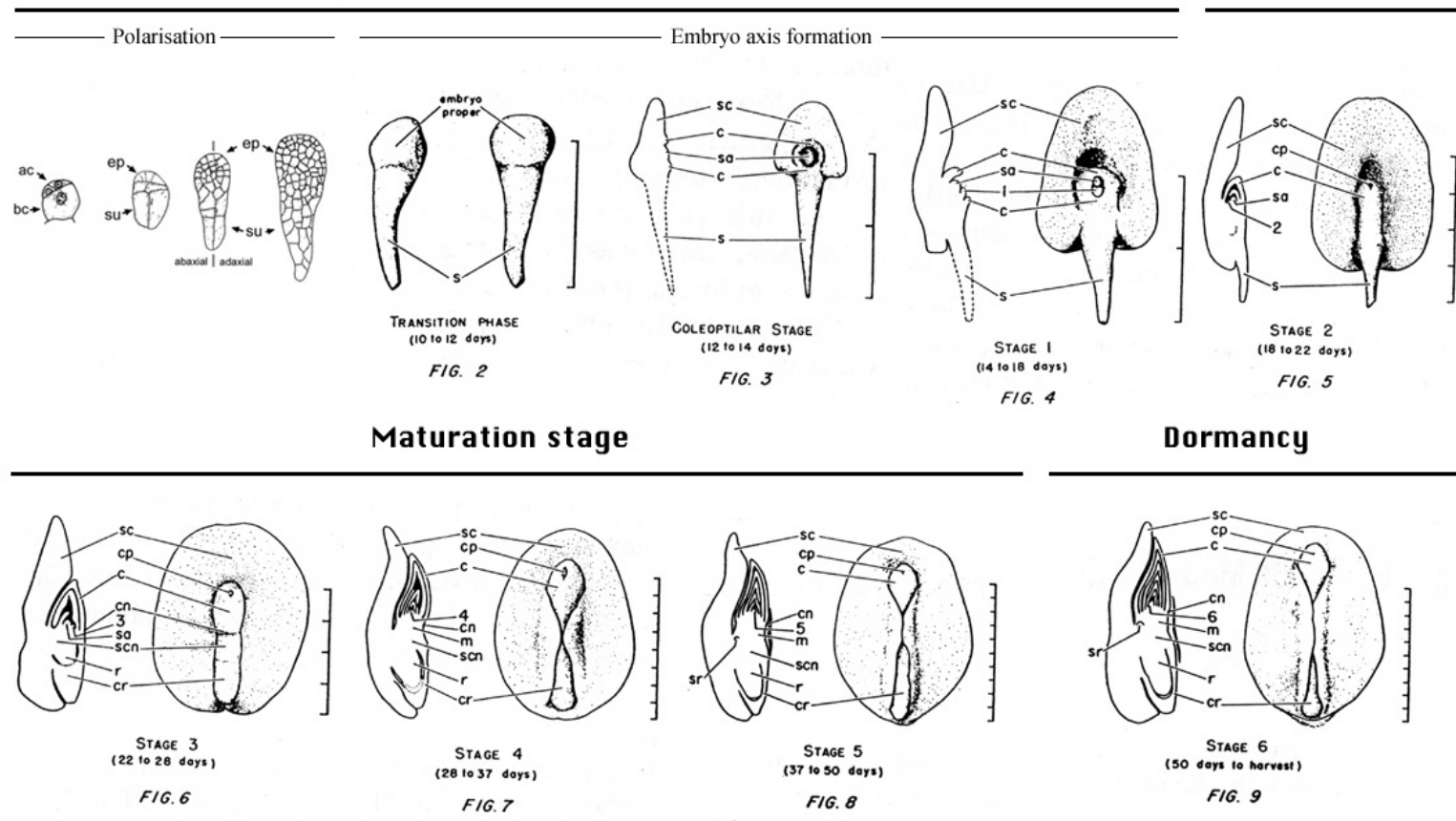

Figure1.2: Schematic representation of the different developmental stages of the Zea mays embryo, showing a longitudinal section (left drawing) and a frontal view (right drawing) for each stage of middle and late embryogenesis. Abbreviations: 1-6, leaf primordia in order of its arise; c, coleoptile; cn, coleoptilar node; $\mathbf{c p}$, coleoptilar pore; cr, coleorhiza; m, mesocotyl; r, root primordia; s, suspensor; sa, shoot apex; sc, scutellum; scn, scutellar node; sr, lateral root primordia. Adapted from Abbe and Stein (1954) with modifications. 
During the following maturation phase the different leaf primordia appear (L2-L6 stage) and storage products are accumulated in the scutellum (L2-L4) (Fig. 1.2). The scutellum grows in size by the accumulation of protein bodies, whereas, the suspensor degenerates and disappears at stage L3. It is also at this stage when the primary root, covered by the coleorhiza, becomes visible. By stages L5 and L6 the embryo has reached its maximum size, and has the characteristics of a little plant. Provascular strands have formed and the root primordia have the anlagen for lateral secondary roots (Sheridan and Clark 1987; Meinke 1995).

The seed looses up to $95 \%$ of its water content in preparation for the dormancy. RNA and protein synthesis decreases and dormancy begins (Sheridan and Clark 1987; Meinke 1995).

While in Arabidopsis nearly the entire endosperm is absorbed during seed development and the nutrients for germination are stored in the cotyledons, in Zea mays the endosperm assumes the more important role of nutrient delivery after germination. Therefore it represents an important part of the seed, in preparation for germination (Lopes and Larkins 1993).

\subsubsection{Genetical approach to study embryogenesis of plants}

All the processes that lead the embryo through the different stages of embryo and seed development have to be controlled by highly co-ordinated genetic regulation in time and space. This is demonstrated by the existence of multiple mutants disturbing these processes in a certain way (Meinke 1995). The study of mutants is the general strategy to identify essential genes for the correct development of the embryo and seed. The question of whether these genes act independently or together and in which way genetical hierarchy is present may be answered by this approach (Meinke 1995). The limitations of the study of mutants are the mutation of genes that are duplicated in the genome or genes required during gametogenesis. The mutation of these genes would not lead to a loss-of-function mutant. And if a loss-offunction mutant is obtained the question still remains, whether the gene is specific for embryo development or if the gene is important during the whole life cycle, and is expressed in very early stages of embryogenesis. Nevertheless, a high number of 
Arabidopsis and maize mutants defective in embryogenesis exist in several mutant collections. The study of these mutants facilitates the study of plant embryogenesis (Clark and Sheridan 1991; Jürgens et al. 1991).

Mutants of Arabidopsis and Zea mays are obtained by chemical mutation (diepoxybutane), by X-ray radiation or by insertion of DNA fragments. These DNA fragments can be T-DNA originated from Agrobacterium tumefaciens in the case of Arabidopsis or an endogenous mobile element, such as the Ac-element (Activatorelement) in the case of Zea mays (Chandlee 1991; Feldmann 1991; Weigel et al. 2000). These latter methods allow for the fast isolation of the mutated gene (Meinke 1991; Meinke 1995; Newbigin et al. 1995).

Several laboratories have characterized mutants of Arabidopsis defective at different stages of embryo development. Most of the studies on these mutants, whose pattern formation was disturbed, followed the model for the study of embryonic development in Drosophila. This approach is based on the theory that a gene involved in pattern formation of the embryo does not necessarily play an important role in basic cellular functions and the further development of the embryo can continue, even with alterations in its structure (Mayer et al. 1991; Meinke 1991; Weigel 1993; Meinke 1995). The identification of this kind of mutant is possible in early stages after germination, because pattern formation takes place very early in development. If the seedling has a defective structure the origin lies probably in the altered embryo formation. Following this criteria the collections of mutants contain multiple mutants with defective patterns before the heart stage. At this stage the embryo pattern in Arabidopsis is already established (Mayer, Torres Ruiz et al. 1991; West and Harada 1993; Jürgens et al. 1994; Franzmann et al. 1995). However, the study of mutant genes should not be restricted to those embryo mutants with altered patterns. Also the study of genes that may interact and connect the development of the embryo proper, suspensor, endosperm, seed coat, nucellus and maternal plant in the developing seed may contribute to the understanding of plant embryogenesis. Thus it is also necessary to study mutants defective in dormancy, germination and the accumulation of storage materials (Meinke 1991; Meinke 1995). 
The development mutants in maize are divided in two groups: the first group includes germless emb mutants (embryo specific), which shows alterations affecting only the embryo. The mutations that alter the embryo as well as the endosperm are classified as dek mutants (defective kernel). All these mutants are lethal, since no germination occurs, even if the mutants disturb the development at different stages and the processes are interrupted at distinct levels (Sheridan 1988; Clark and Sheridan 1991; Sheridan and Clark 1993; Meinke 1995; Heckel et al. 1999). The data obtained by the study of the maize mutants are applicable in other cereals of agronomic interest such as rice, barley, wheat and sorghum (Meinke 1995; Bommert and Werr 2001). However, the maize model has limitations that complicate the work with this species. The life cycle of Zea mays is long and cultivation needs extensive space. In addition, until now, no effective working system for the transformation of maize had been established. The multiple endogenous transposons in the maize genome complicate genetic studies and few alleles are available for the known and described mutants. In the study of embryogenesis and pattern formation the variable division plan during embryo development implies additional difficulties for the study of maize embryogenesis.

\subsubsection{Molecular approach to study embryogenesis of plants}

The application of methods of molecular biology to obtain information about the molecules and genes that control and coordinate embryo formation is important for the understanding of the mechanisms of embryogenesis. This includes the isolation and characterisation of genes whose mutation results in defective embryos and seeds, because these genes are directly related to correct development. But also, genes that are not exclusively related to development, are helpful tools as marker genes to study embryogenesis and are used to identify specific organs, the stage of differentiation of certain tissues or to monitor the course of development (Goldberg, de Paiva et al. 1994). The presence of a marker gene can announce the initiation of differentiation, even before the consequences of the gene action can be observed histologically. This is a useful feature of molecular markers, since at the cellular level gene expression often starts just before differentiation takes place (West and Harada 1993; Goldberg, de Paiva et al. 1994). 
Several marker genes are known, covering different aspects of embryo development. The expression of these marker genes provides information about metabolic processes, the cell cycle, the accumulation of storage proteins, the desiccation program, or identify specific tissues or organs (Meinke 1995). These marker genes become especially important in the characterisation of mutant phenotypes. Sometimes the resulting phenotypes are so severe that it is not possible to determine the state of development or the tissue organisation and identity. A marker gene can also provide information about the type of process that is interrupted or disturbed by the mutation (Topping and Lindsey 1997; Topping et al. 1997).

A variety of techniques are applied to isolate genes or determine specific marker genes, such as the differential screening (Meinke 1995) and differential display (Liang and Pardee 1992).

\subsection{Pattern formation during plant embryogenesis}

From the morphological point of view, the period of embryogenesis is of most interest, since the basic organisation of the plant body is established during this period (Randolph 1936; Sheridan and Neuffer 1982; Clark and Sheridan 1991; Laux and Jürgens 1997). These basic structures comprise the formation of an apical-basal axis and the establishment of a radial pattern. The morphogenic processes and pattern formation occur along these two perpendicular oriented axes (Jürgens 1995; Hudson 2000).

\subsubsection{The apical-basal axis}

The apical-basal pattern is defined by the shoot meristem, the cotyledons, the hypocotyl, the root and the root meristem and can be separated in three embryonic regions: apical, central and basal (Jürgens 1995; Souter and Lindsey 2000) (Fig. 1.3 A,B). In the case of Arabidopsis these regions are determined in the late proembryo stage or 8-cell-stage and each region follows its own programme of cell division. As shown in Figure 1.3 C,D the early regions do not correspond to the seedling primordia structure (Jürgens 1995). The apical region gives rise to the shoot meristem 
and most cotyledons, while the central part contributes to the cotyledon, the hypocotyl, the root and the root meristem. From the basal region arise only the columella initials, the quiescent centre and the central root cap (West and Harada

Figure 1.3: Formation of the ApicalBasal Axis in the Arabidopsis Embryo. (A) Asymmetric division of the zygote, giving a small apical (a) and a large basal (b) cell. (B) The 8cell stage. The proembryo (proE) consists of two tiers each of four cells (regions $\mathrm{A}=$ apical; $\mathrm{C}=$ central; $\mathrm{B}=$ basal) and is connected to the founder cell of the basal region of the embryo. (C) Embryo at heart stage. Approximate locations of cell groups that give rise to the primordia of seedling structures are indicated.

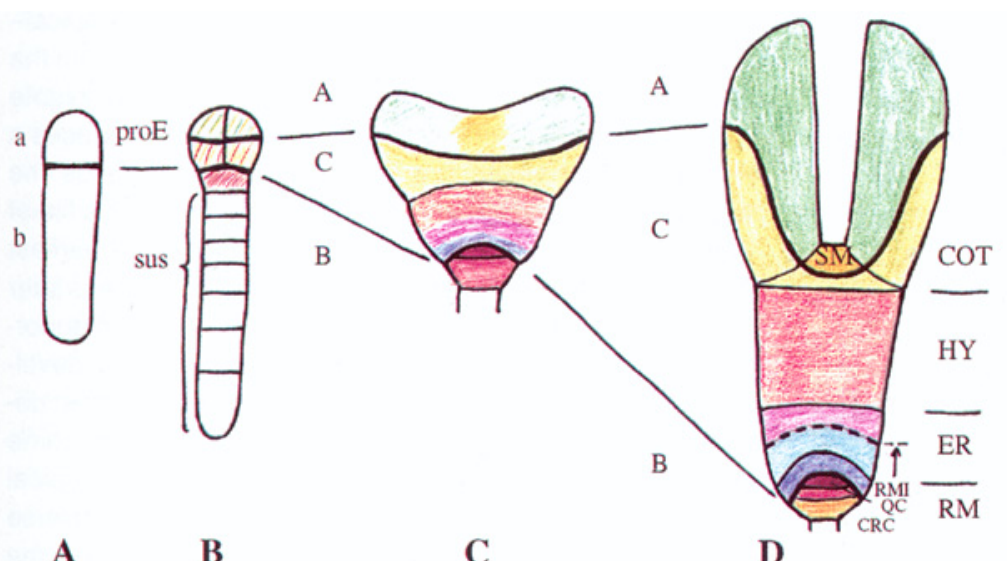

A $\quad$ B
C

(D) Embryo at Torpedo stage. Clonal boundaries are marked by thick lines. The broken line indicates the upper end of the embryonic root derived from the root meristem initials (RMI). Below the quiescent center (QC) of the root meristem are the initials of the central root cap (CRC). Primordia of seedling structures: COT, cotyledons; HY, hypocotyl; ER, embryonic root; RM, root meristem; SM, shoot meristem.

Adapted from Jürgens (1995) with modifications.

1993; Souter and Lindsey 2000). The conclusion that cell ancestry plays a dominant role in apical-basal pattern formation, was based on these observations (Goldberg, de Paiva et al. 1994). Furthermore, the regularity of cell division in the early Arabidopsis embryo is deceptive, since mutations in the FASS (FS) gene totally alter the pattern of cell division, without affecting pattern formation (Torres Ruiz and Jürgens 1994).

The study of several Arabidopsis mutants including gurke (GK) (Torres Ruiz et al. 1996), fackel (FK) (Mayer, Torres Ruiz et al. 1991), monopteros (MP) (Berleth and Jürgens 1993), gnom (GN) (Mayer et al. 1993; Meinke 1995) and bodenlos (BDL) (Hamann et al. 1999), which are defective in exclusively one or two early regions of the apical-basal pattern (Fig. 1.4), provide evidence that the early regions define cell groups expressing different genes which are important for the development of each region. Thus, gurke mutants do not form cotyledons and fail in the formation of a shoot meristem, while in fackel mutants no hypocotyl tissue separates the apical from the basal region. The gnom mutants lack apical and basal domains. More detailed characterisation of these mutants revealed that the mutation first affected the corresponding region, but subsequently the effect was observed in the neighbouring 
cells (Hudson 2000; Souter and Lindsey 2000). These results suggest that the apical basal pattern elements are established by cellular interactions in a position-dependent manner and that this positional information allows cells to activate a co-ordinated and differential gene expression (Jürgens 1995; Mayer and Jürgens 1998; Hudson 2000; Souter and Lindsey 2000; Jürgens 2001).

\begin{tabular}{|c|c|c|c|c|c|}
\hline WT seed & WT seedling & emb 30/gnom & monopteros & gurke & fackel \\
\hline Complete & Complete & $\begin{array}{l}\Theta \text { Apical } \\
\Theta \text { Basal }\end{array}$ & $\begin{array}{l}\Theta \text { Central } \\
\Theta \text { Basal }\end{array}$ & $\Theta$ Apical & $\Theta$ Central \\
\hline
\end{tabular}

Figure 1.4: Schematic representations of Arabidopsis pattern mutants. The green, yellow and orange colours delineate the apical, central and basal regions, respectively. The lacking regions are indicated. Abbreviations: WT, wild type; RM, root meristem; SM, shoot meristem; C, cotyledon; $\mathbf{h}$, hypocotyl; R, root. Adapted from Goldberg et al (1994).

Molecular characterisation of the monopteros mutants identified the $M P$ gene as a transcription factor, which possesses the same binding specifity to auxin-responsive promoter elements as AUXIN RESPONSE FACTOR1 (ARF1) (Ulmasov et al. 1997; Hardtke and Berleth 1998) and is required for cell axialization during early embryogenesis and for post-embryonic vascular development (Hardtke and Berleth 1998). For that, $M P$ is assumed to play a role in regulating the transcription of auxin responsive genes (Souter and Lindsey 2000). Moreover, other embryo defective mutants may be related to the incorrect processing of auxin (Jürgens 1995; Hudson 2000; Souter and Lindsey 2000).

\subsubsection{Establishment of the radial pattern}

The formation of the protoderm by anticlinal cell division parallel to the surface of the embryo proper is the first histological evidence for the differentiation of a tissue layer and the initiation of the establishment of a radial pattern (Fig. 1.5). The formation of the inside-out asymmetry occurs early in embryogenesis (Arabidopsis eight-cell stage; Zea mays - transition stage) and the basic structures are defined 
before the heart stage (Randolph 1936; Jürgens 1995; Hudson 2000). The protoderm forms the epidermis by periclinal divisions, while the inner cell mass separates as ground tissue and the central vascular primordia (Fig. 1.5B). The latter divides again in a periclinal manner to give rise to the pericycle layer (Fig. 1.5C) and results in a hypocotyl and a root consisting of concentric cell layers that appear radially symmetrical in transverse sections (Fig. 1.5C,D) (Jürgens 1995; Laux and Jürgens 1997; Hudson 2000).

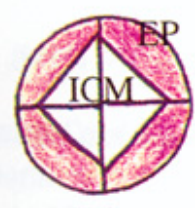

A

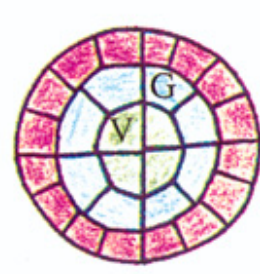

B

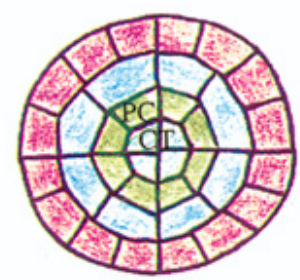

C

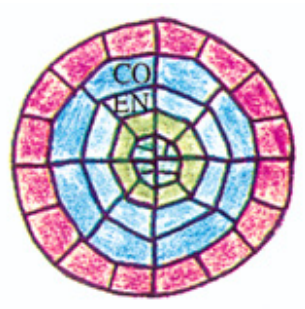

D

Figure 1.5: Formation of the Radial pattern in the Arabidopsis Embryo. Schematic cross section through the central region of embryo (A and $\mathbf{B})$ and through the root primordia $(\mathbf{C}$ and $\mathbf{D})$. (A) proembryo: $\mathbf{E P}$, outer epidermis layer; ICM, inner cell mass. (B) Globular embryo: $\mathbf{G}$, ground tissue; V, vasculated primordium. (C) Heart stage: PC, pericycle; CT, conductive tissue. (D) Torpedo stage: CO, outer cortex; EN, inner endodermis layer. From Jürgens (1995) with modifications.

The ARABIDOPSIS THALIANA MERISTEM LAYER1 (AtML1) gene in Arabidopsis and the LIPID TRANSFER PROTEIN2 (LTP2) gene from Zea mays are specific marker genes for the peripheral cells of the embryo proper. These genes are also used to distinguish apical and basal hemispheres of the pro-embryo (Hudson 2000; Bommert and Werr 2001). The information provided by the gene expression of the LTP2 gene and the protoderm specific genes of the ZEA MAYS OUTER CELL LAYER (ZmOCL) family (Ingram et al. 1999) suggests that the formation of the radial pattern occurs in at least two steps and that the embryo proper is regionalized (Bommert and Werr 2001). The latter assertion is based on the overlapping gene expression of ZmOCL4 and ZmOCL5, which determines an adaxial and an abaxial region of the embryo proper (Fig. 1.6) (Ingram et al. 2000; Bommert and Werr 2001). Also the discontinuous gene expression of LTP2 in the epidermis suggests this spatial regionalization (Fig. 1.6) (Bommert and Werr 2001). 

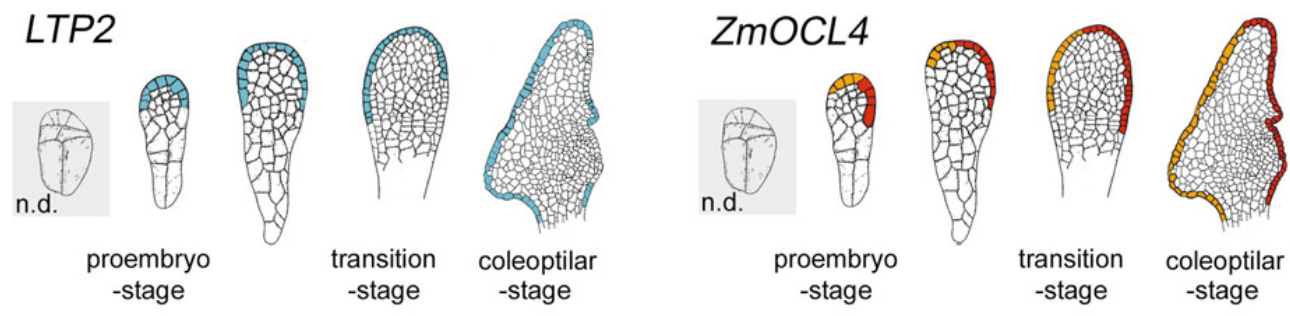

ZmOCL1
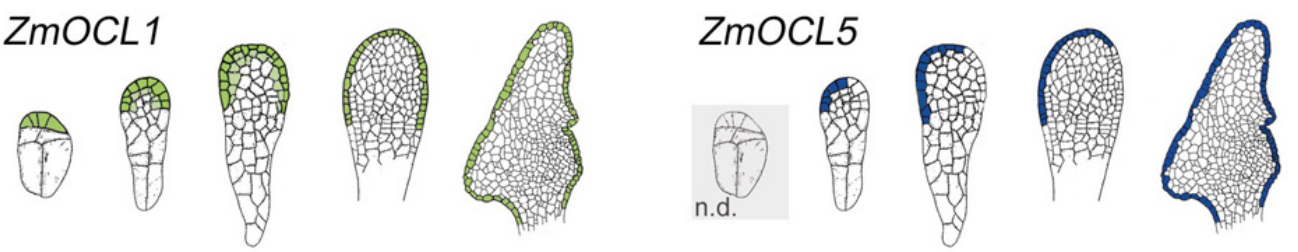

Figure 1.6: Expression domains in longitudinal medial sections of proembryo-, transition, and coleoptilar-stage maize embryos. The adaxial face is oriented to the left. LTP2 expression (light blue) restricted to one layer, but not continuous. ZmOCL1 expression (green) during proembryo-stage homogenous changes to a one-layer expression in the transition- and coleoptilar-stage. ZmOCL4 and ZmOCL5 expression with ad- and abaxial preferences; orange $=$ weak expression; red=strong expression. n.d. = not detected; Adapted from Bommert and Werr (2001) with modifications.

The studies on Knolle and Gnom mutants showed that the inside-out asymmetry is not dependent on their apical-basal symmetry. The Gnom mutants, as mentioned before, are disturbed in apical-basal polarity, however they produce a normal protoderm expressing the marker AtLTP1 (Vroemen et al. 1996). In accordance with this, Knolle mutants have defects in the inside-out pattern but retain aspects of apical-basal asymmetry (Mayer, Torres Ruiz et al. 1991; Hudson 2000).

\subsection{The role of auxin and polar auxin transport in embryogenic and post-embryogenic pattern formation}

In the previous sections the cell-to-cell-communication and the signalling between different embryonic regions was underlined as an essential condition for the establishment of apical-basal and inside-out asymmetry. Moreover, precise positional information is necessary for embryonic development (Jürgens 1995; Hudson 2000; Souter and Lindsey 2000).

Many studies of the pattern formation process indicate that auxin is an essential signaling molecule to provide positional cues for correct spatial patterning 
(Uggla et al. 1996; Sabatini et al. 1999; Doerner 2000). Most of these studies about how auxin flux and auxin levels control cell division and cell differentiation were carried out in Arabidopsis roots, because of its stereotyped division patterns and developmental trajectories (Sabatini, Beis et al. 1999). But also a number of Arabidopsis embryo mutants, described in parts in the previous sections, give evidence for auxin action in the very early stages of development. The effect on the phenotypes is diverse, from fused cotyledons (pin1-1), and lack of body parts (monopteros, bodenlos) to an arrest in development and lethality (gnom, abp1) (Berleth and Jürgens 1993; Liu et al. 1993; Mayer, Büttner et al. 1993; Hamann, Mayer et al. 1999; Chen et al. 2001 respectively). The defective genes of these mutants are also necessary in the postembryonic wild type plant and can be linked directly or indirectly to auxin transport or reception. Moreover, some of the mutant phenotypes can be phenocopied by inhibition of auxin transport (Liu, Xu et al. 1993; Fischer and Neuhaus 1996; Hadfi et al. 1998). The cultured embryos of Brassica juncea (Indian mustard) and Triticum aestivum (wheat) were exposed to several auxin transport inhibitors as 9hydroxyfluorene-9-carboxylic acid (HFCA), trans-cinnamic acid and 2,3,5triiodobenzoic acid (TIBA). The result of these experiments shows a lack of bilateral symmetry in the treated embryos as observed in the mutants mentioned before (Liu, Xu et al. 1993; Fischer and Neuhaus 1996; Hadfi, Speth et al. 1998). Also the addition of exogenous auxin in high concentrations revealed an inhibitory effect on embryonic development leading to developmental arrest in the early transition stage (Fischer and Neuhaus 1996).

Auxins are plant hormones with an influence on a wide variety of processes in the plant, such as cell division, cell elongation, cell differentiation and the initiation of organ formation (Davies 1995). Auxins control mechanisms of tropism (Estelle 1996; Dolan 1998; Palme and Galweiler 1999), vascular strand formation (Sieburth 1999; Berleth and Mattsson 2000; Berleth et al. 2000), lateral root (Reinhardt et al. 2000) and flowering initiation (Okada et al. 1991; Oka et al. 1999), apical dominance and senescence (Guilfoyle et al. 1998). In spite of the 70 years of auxin research little is known about how a molecule as simple as the most common auxin, indole-3-acetic acid (IAA), controls such a variety of finely regulated responses within an assortment of cells and tissues in dimensions of space and time. Only in the last few years the 
first insights have been achieved to understand auxin perception, signal transduction and gene expression (Guilfoyle, Hagen et al. 1998).

The characterisation of the auxin binding proteins (ABP1) from Zea mays (Lazarus et al. 1991; Napier and Venis 1995) and Oryza sativa (Kim et al. 1998)) as well as the identification of cis-acting elements (DNA sequences that confer auxin responsiveness to a promoter) and trans-acting factors (i.e. transcription factors that bind to the cis-acting elements) gave clues how endogenous or exogenously applied auxin induces a rapid expression of defined genes (Abel and Theologis 1996; Guilfoyle, Hagen et al. 1998). Promoters from genes such as PS-IAA4/5 from Pea (Ballas et al. 1993; Ballas et al. 1995) as well as the soybean genes GH3 (Liu et al. 1994; Ulmasov et al. 1995) and SAUR15A (Li et al. 1994; Xu et al. 1997) contain auxininducible cis-acting elements (or auxin-responsive-elements, AuxREs) and are rapidly and specifically activated by biological active auxin. In Arabidopsis nine full-length trans-acting factors (or auxin-response-factors, ARF) have been identified, which specifically bind to AuxREs, representing a family of transcription factors (Guilfoyle et al. 1998). ARF5 has been showed to be identical to the gene product of the MP gene (see section 1.2.1) (Berleth and Jürgens 1993; Hardtke and Berleth 1998) whose inactivation by mutation causes defects in the axis formation of the embryo and the development of vascular strands. The mutant seedlings fail in the development of hypocotyl and root, and show phenotypes that can be phenocopied by plants treated with auxin-transport inhibitors (Przemeck et al. 1996).

\subsubsection{Polar auxin transport}

One of the outstanding features of auxin is its polar translocation from the sites of biosynthesis (young leaves and meristematic tissues), passing through the whole plant to the root tip (Fig. 1.7A). The basipetal flow in the shoot to the shoot-root junction occurs mainly through the vascular tissue (Jones 1998). In the root itself there are two pathways with different directions. First, the acropetal (from the shoot-root junction to the root tip) through the central stele, and second the basipetal (from the root apex to the shoot-root junction) through the outer cell layers of the root (Jones 1998; Muday and DeLong 2001). These two flow directions seem to be important for the regulation of two distinct processes. The acropetal movement is necessary for the 
initiation of lateral roots, while the basipetal flow is required for the gravity response of the root (Reed et al. 1998; Rashotte et al. 2000).

To explain the molecular basics of the polar transport of auxin, Rubery and Sheldrik (1974) and Raven (1975) proposed, independently, a model termed the chemiosmotic model of polar auxin transport (PAT), postulating that auxin enters and leaves the cell by carrier mediated transport (Rubery and Sheldrake 1974; Raven 1975). The molecule (i.e. IAA, the most common free auxin in the plant) enters from the apoplast $(\mathrm{pH} 5,5)$ into the cell in a protonated form. Inside the cell the $\mathrm{pH}$ is about 7.0 and the anionic form of IAA is favoured under these conditions. The negatively charged IAA accumulates inside the cell. This accumulation effect is known as the "auxin trap"(Fig. 1.7B). The way out of the cell is mediated by an efflux carrier system, sensitive to synthetic inhibitors of auxin transport, including $\mathrm{N}$ naphthylphthalamic acid (NPA) and tri-idobenzoic acid (TIBA), localized at the basal end of the cell, providing the polarity of auxin flux. After a long time without any clues about the molecular components and regulation mechanisms of PAT, the characterisation of the proteins AUX1 (Marchant et al. 1999), PIN1 (Gälweiler et al. 1998) and EIR1 / AGR1/ PIN2 (Chen et al. 1998; Luschnig et al. 1998; Müller et al. 1998; Utsuno et al. 1998) from Arabidopsis identified these proteins as putative members of the auxin transport system. Meanwhile AUX1 is the only described auxin influx carrier (Marchant, Kargul et al. 1999), PIN1 and EIR1 (EIR1, AGR1 and PIN2 are the same proteins) encode putative auxin efflux carriers and represent two of ten members comprising the PIN gene family (Estelle 1998). With ten membrane spanning domains in two groups of five, separated by a hydrophilic region and a certain homology to bacterial membrane transporters, PIN1 and EIR1 fulfil the criteria for integral membrane transporters. Immunohistochemistry on Arabidopsis roots showed an asymmetric localization in the plasma membrane for PIN1 and EIR1 (Gälweiler, Guan et al. 1998; Müller, Guan et al. 1998). In addition, mutants of pin1 and eirl exhibit abnormal auxin transport in the inflorescence and root and show alterations in the phenotypes, consistent with disturbed auxin distribution (Chen, Hilson et al. 1998; Gälweiler, Guan et al. 1998; Luschnig, Gaxiola et al. 1998; Müller, Guan et al. 1998; Utsuno, Shikanai et al. 1998). The NPA inhibitory effect is not yet resolved, since a direct NPA binding to the transporting protein or substrate 
competition have been excluded. Nevertheless, the evidence for an additional polypeptide with NPA binding ability exists, termed NPA-binding protein (NBP) (Sussmann and Gardner 1980; Muday 2000). This polypeptide is possibly located close to the transporting protein and may assume a regulatory function over the efflux carrier (Luschnig 2001; Muday and DeLong 2001) (Fig. 1.7B). The mutants of transport inhibitor response3 (TIR3) (Ruegger et al. 1997) and dark overexpression of cab (doc) (Li et al. 1994) are defective in genes, which are suggested to be other components of the auxin efflux machinery. These two mutants were found independently without any direct relation. Finally they were identified to be two alleles of the same gene, which encodes a protein of exceptional size, termed BIG (Gil et al. 2001). The localization of PIN1 in NPA treated tir3/doc1 mutants results in an accumulation of the putative auxin carrier PIN1 in inner cell compartments in contrast to wild type plants, where PIN1 localizes normally in the presence of NPA (Gil, Dewey et al. 2001; Luschnig 2001). Based on these results the authors indicate a possible role of BIG in the regulation of PIN1 positioning (Gil, Dewey et al. 2001).

Although several proteins have been identified, which might be components of the auxin transport system and its regulatory mechanism, questions such as how PAT

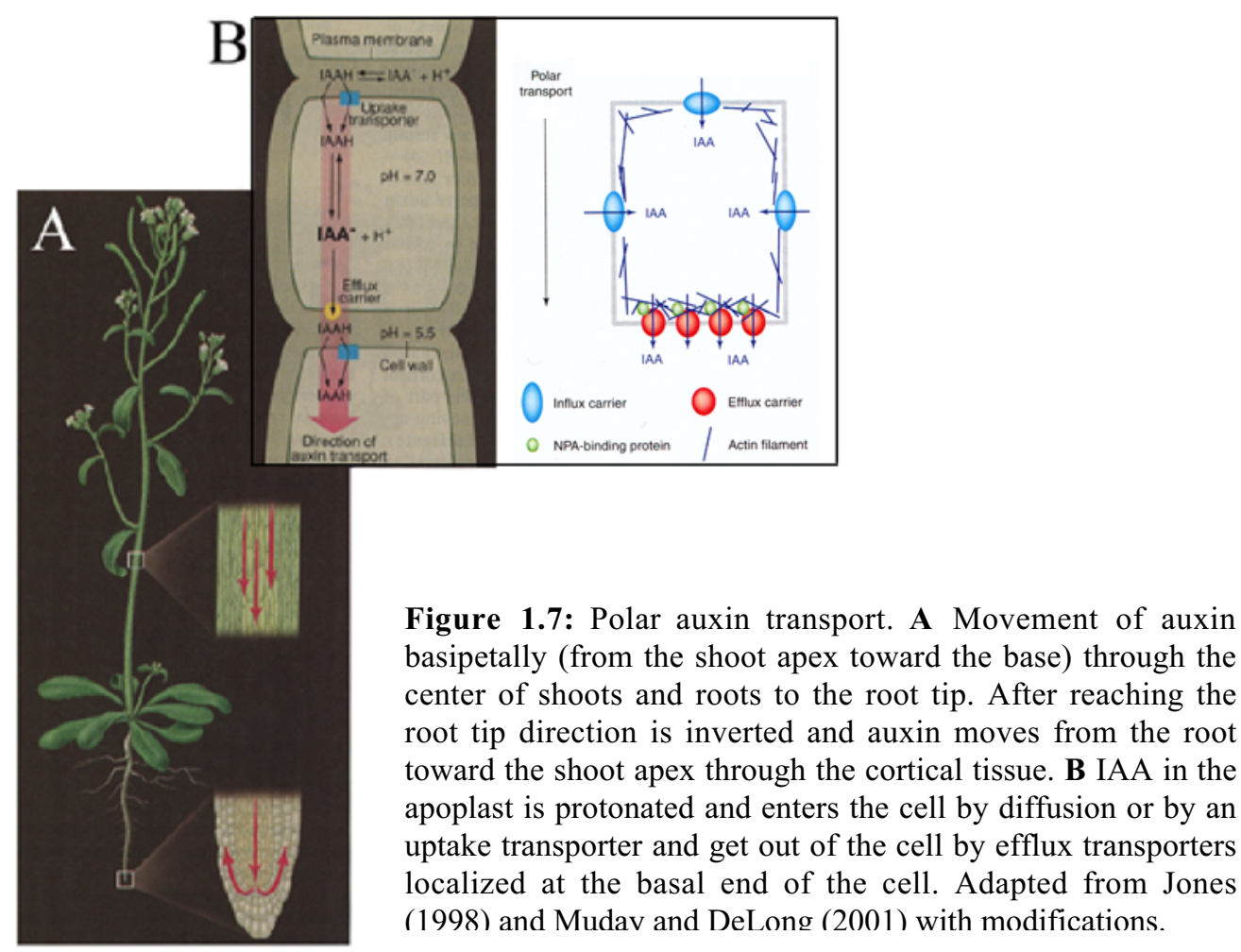


inhibitors act and which proteins are the targets for the regulating actions of kinases and phosphatases remain unanswered (Muday and DeLong 2001). Also lacking are data from convincing physiological assays that may confirm the auxin transport capacity of the putative transporter proteins AUX1, PIN1 and EIR1 (Jones 1998; Muday and DeLong 2001).

Another important question concerning polar auxin transport is the way the presumed auxin carriers are localized in the cell membranes. Experimental data suggest the involvement of localized vesicle targeting in the localization process of efflux transporters (Morris 2000). This assumption is confirmed by the inhibitory effect of the drugs monensin and brefaldin A (BFA) on Golgi vesicle secretion. The presence of monensin reduces auxin efflux activity and experiments with BFA point to a relation between auxin efflux and vesicle cycling (Delbarre et al. 1998; Morris and Robinson 1998). Studies on the effects of BFA and cytochalasin D (a drug that fragments actin filaments) on the localization of PIN1 provide a direct link between vesicle targeting and polar localization of PIN1 (Steinmann et al. 1999; Geldner et al. 2001). PIN1 is not positioned at the basal end of the cell in the presence of any of these drugs, nor in the gnom mutant, where an inactivated GNOM gene product is not able to regulate vesicle trafficking (Steinmann, Geldner et al. 1999; Geldner, Friml et al. 2001). There are several possible interpretations of these data. More experimental data is necessary to clarify the network between NPA action, vesicle traffic and auxin transport components. The data indicate that actin filaments might also be required for the initial polar localization of auxin transport components and also for the subsequent vesicle-mediated cycling of efflux carriers (Estelle 2001; Geldner, Friml et al. 2001).

The data discussed in the previous sections are results of experiments in Arabidopsis since little is known about the auxin transport system in other species such as maize, rice or potato. But assuming the omnipresence of auxin and the essential role that these molecules play in plants, the mechanisms of auxin perception, metabolism and transport are likely to be well conserved. 


\subsection{The maize defective kernel mutant lachrima from Zea mays}

The dek mutant lachrima from Zea mays was isolated by Ac-transposon tagging during a screen for mutants defective in embryo development. The phenotype of lachrima has a small mature kernel with a collapsed endosperm and a teardrop shaped embryo that is uniformly blocked in the mid-transition stage without developing bilateral symmetry (Fig. 1.8). The analysis of the recessive embryo-lethal mutant lachrima showed that an Ac-element inserted in the promoter region inhibits the expression of an unknown gene called TM20. The isolation of the full coding sequence of TM20 lead to a gene of $4.4 \mathrm{~kb}$ that is expressed during the first 25 days after pollination (DAP) and Southern analysis identified the gene as a single copy gene.

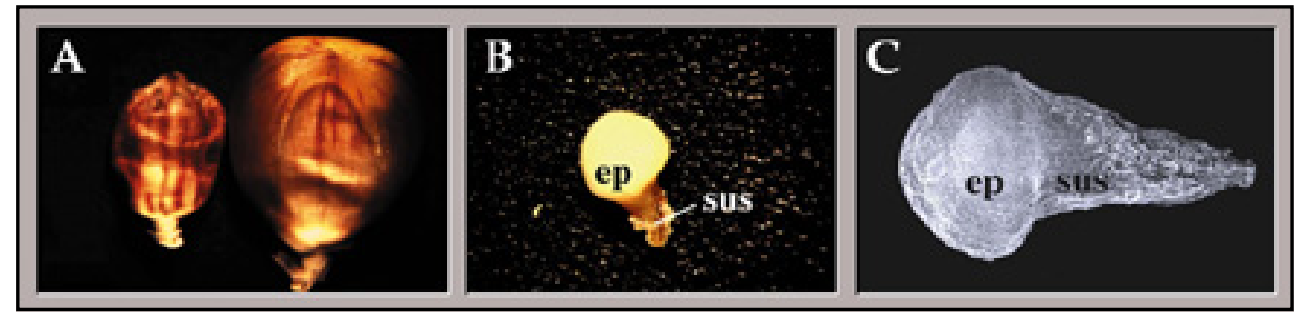

Figure 1.8: Phenotypes of lachrima. A: collapsed lachrima kernel (left) next to a wild-type kernel (right); B: fresh dissection at maturity of a lachrima mutant; $\mathbf{C}$ : scanning electron microscopy of a lachrima embryo at kernel maturity. The diameter of the embryo proper is $1 \mathrm{~mm}$. ep = embryo proper; sus = suspensor. Adapted from Stiefel et al (1999) with modifications.

The in situ hybridization of TM20 mRNA in embryo tissue showed that TM20 is expressed at very early stages of kernel development and can be found in the proembryo and the endosperm. As development proceeds the expression of TM20 becomes more restricted to the embryo. At about 12DAP to 20DAP RNA accumulates exclusively in the embryo, where the major expression is detected in meristematic tissues of the embryo. TM20 gene expression seems to be associated with proliferating tissues such as the provascular cells of the leaf primordia and the coleoptile in the shoot. In the node and the root primordia RNA of TM20 is also detected in the provascular cells and in proliferating tissue such as the pericycle (Fig. 1.9) (Stiefel et al. 1999). 
The deduced amino acid sequence of 1389 aa encodes a protein with a molecular weight of approximately 152 KDa (Fig. 1.10). The analysis of the deduced amino acid sequence indicated 20 hydrophobic domains grouped together in five homologous arrangements of four hydrophobic domains, with a central hydrophilic region between the second and the third arrangement. The hydrophilic regions exhibit the properties of transmembrane spanning elements, suggesting a membrane integral localization for the TRANSMEMBRANE 20 (TM20) protein. On the end of the third transmembrane segment of every group a phosphorylation site for kinase $\mathrm{C}$ was identified that may play a role in the regulation of the activity of TM20 (Stiefel, Becerra et al. 1999).

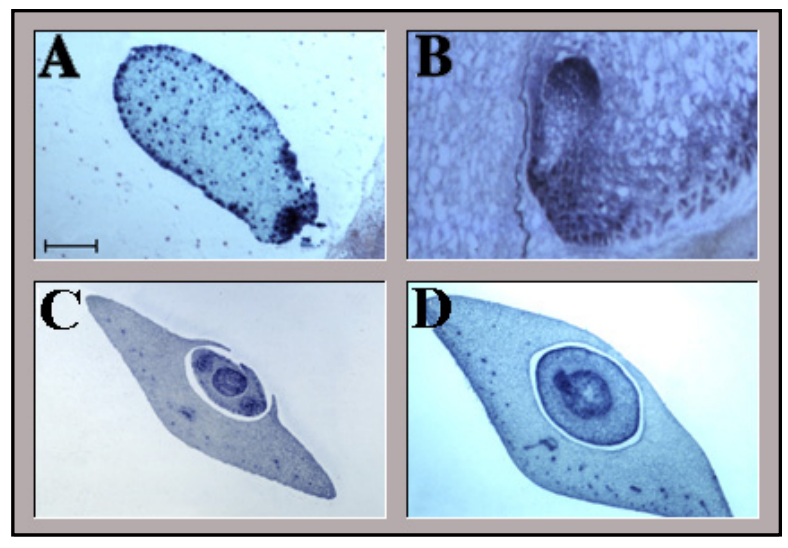

Figure 1.9: TM20 gene expression in embryo sections detected by in situ hybridization. A: longitudinal sectionof a 4DAP kernel. B : longitudinal section of $8 \mathrm{DAP}$ kernel. C: transversal section of the apical region of a 20DAP embryo. D: transversal section of a 20DAP embryo at root level. Adapted from Stiefel et al (1999) with modifications.

No protein similar to TM20 in sequence or structure could be found in the databases, so it was suggested that TM20 is a new class of membrane protein with an unknown function.

The phenotype of lachrima is blocked between the pro-embryo and the transition stage and lacks the formation of bilateral symmetry. This characteristic has been described for the embryo mutants defective in genes, which are implicated in the establishment of the auxin gradient (Liu, Xu et al. 1993). Moreover, it has been shown that wheat embryos cultured in the presence of auxin transport inhibitors or an excess of exogenous auxin are blocked in their development at the same stage as the lachrima mutant is blocked (Fischer and Neuhaus 1996). These data point to the essential role that auxin plays in the establishment of bilateral symmetry and the development of the embryo. These results together with the phenotype of the lachrima mutant and the protein structure of TM20 suggests that TM20 could be involved in a mechanism 
necessary for the establishment of a correct auxin gradient in the plant embryo (Stiefel, Becerra et al. 1999).

MSYTHAHGLI IDLDVVVCVCVGSFSLSFLEINIVLAFQRVFSLRSSLGALTSPKSSSSFFGSCQFHTPLFLSRSILGFLF IPDTSSLKPKTPCSLFFRCIVGEGSFTCEAEVSNHCWRASSHMASTAATPEAGSNHSEDGTTVELPIVVTGSTSQESIDT

161 PSTRYLLAPSHVSSSSFTADIELLWRLRKYLLLLGILAVGVTYNAGLTPPGGFWSKNTQGQSGHEAGDPVLRALFFPRHE

241 VFFYCNATAFAASLVLIILLLSKNVARORLWLRSMOLTMVLDLFSLMGAYAAGSCRAVKSSIYIWVLVLSVFTYIMIHIL

321 VFMKVVPRFVSEKRFVPKRLKDVARSVERWILSRCGVHRSEKNSSHEKDLEEARKFTLVLVTFAATVAYQAGLSPPGGFW

401 AENDENKTPATSMLRSGNLPRYNTFVVCNSTSFVASLVTIILLLSPELSRHGIRSRAVTVCVVVDILGLVGAYAAGSCRS

481 VVTSVSAVLVAVLVWICFAVLAGIFVNRSVAEWFGKKIKPDIMRCIDRFGRVF SSNHGRKRSRNPEGENS IASHQQTEES

561 IKGEAEAETARVPEYQLPYHQLAPDIEEGECPGEQQSPGKQQPTNIEVVSISEHASVNEKQAENSSSVMCKLGSOSTDPN

641 SAANEAMTETETGDPFMIFSEVQMLIPICLTLTLNPAENIQDANMEEQQSSLVDGLKTPTTVAGMSNHEHQSVDNHVVQN

721 LIRQTFSTEDQESTTVECLSDIAPNNHNGATNSFKEEKEASEQHLQANEIESFRTNNVARPVENGNVGMYEVTPRQDDGD

801 VNAGANPTDEHLKKSRTYLLLLAILAVSLAYQSGLNPPGGFWTORGTNNSPKSTHHRPYHLPGDPILEDTRHRRYIAFFY

881 LNAIAFVASLVMI IMLLNRRMSYKGI KRYALQMAMIVDLLALTGSYIMGSSRGTKSSIYIWLLVCLVLVYVAVHVLIATH

961 VIPEGCKKAVAQKIENFSCRYIWTKASFRNRGIDGNGSDCEAGQSQRSDADDKTWERRRNLLLMLAVLAATVTYQAGINP

1041 PGGVWSDDSSASGKPGDPILQHNNSKRYDVFYYSNSLSFVASVVITILLVNKESCEHGIKSYALRVCLVVGLVSLLIAYS

1121 AGSSRKARESIYLIVIAVAVLIALVIQVLLLSCTQDSLRGPTGQFIERLLQLLFGTDKAWHGDTSKOKESSGRPEKKVRK

1201 RHKYLMLLAVLAASITYQAGLNPPGGFWSDDNEGHVEGNPGLKPPGALWSDNKGHLAGNPVLLDINPRRYEIFFCFNSIS

1281 FMASIVVVMFLLNKSARKKAVPLEVLHLIMILDLLALMTAFAAGSCRKFRTSVYVYGLVLGVVVYLVIAVLLSSGIAKCL

1361 RPMDRNRVSSQRSPIRAPTASTRIPGDHA 1389

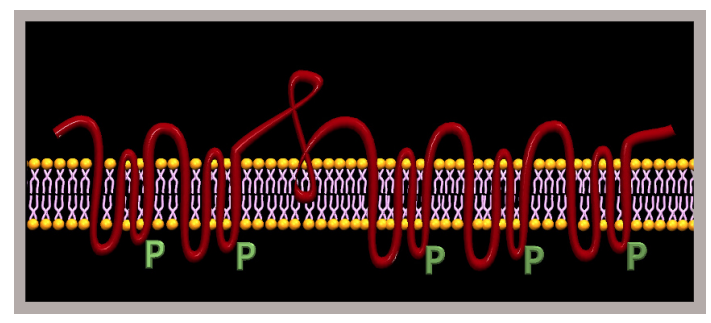

Figure 1.10: Deduced amino acid sequence of TM20. The predicted transmembrane spanning domains are underlined. The predicted phosphorylation sites for kinase $C$ are highlighted in light gray. In red are marked the 5 amino acid sequences that can be recognized by the antibody $\alpha$-loopin 4 (see results 3.2.1.2). A hydrophobic region in the N-terminal important for the insertion of TM20 into the membrane is underlined and in bold. The central hydrophilic region is written in bold. At the panel below a hypotetical model of TM20 is displayed 


\subsection{The aim of the present study}

In the present study the function of the maize protein TM20 was investigated following the hypothesis that TM20 may be involved in auxin transport. Three approaches have been chosen to acquire more information about the function of TM20. First, an extended search in the databases for proteins similar to TM20 was performed to identify information of the presence of TM20 in other species, based on in silico analysis. The second approach comprises the localization of TM20 in embryo tissue and at the subcellular level. For that purpose, specific antibodies against specific zones of TM20 were generated and subsequently used for the localization of TM20 by western blot analysis and by immunohistochemistry. And third, to demonstrate the possible function of TM20 as an auxin transporter, the entire sequence was cloned in an expression vector with the aim of expressing TM20 in oocytes from Xenopus laevis, a heterologous system, and to perform transport assays. 

Material and Methods 


\section{Material and Methods}

\subsection{Biological Material}

\subsubsection{Plant material}

All the plant material, Arabidopsis and Zea mays, was provided by the greenhouse installations of the Centre d'Investigació i Desenvolupament de Barcelona.

\subsubsection{Maize}

The maize material used in this work pertains to the american Zea mays line $\mathrm{W} 64 \mathrm{~A}+/+$. The maize mutants of lachrima are originated in the Lab of Steve Dellaporta from Yale University by Ac-tagging.

To obtain plant material few days after germination, maize kernels were sterilized and germinated embedded in wet filter paper and grown first in the dark and later under the following conditions: 16 hours of light at $26^{\circ} \mathrm{C}$; 8 hours of darkness at $24^{\circ} \mathrm{C}$.

Sterilization of the maize kernels:

1. Wash the maize kernels with $500 \mathrm{ml}$ of autoclaved Mili-Q water and $1 \%$ calcium hypochlorite (Sigma) on a magnetic steerer for $10 \mathrm{~min}$.

2. Four wash steps with autoclaved Mili-Q water on a magnetic steerer for $10 \mathrm{~min}$ each

\subsubsection{Arabidopsis}

The material of Arabidopsis thaliana used in this work pertains to the subspecies Columbia. First the seeds were sowed on agar plates and were transferred after approximately 10 days to soil.

\subsubsection{Bacterial strains}

Several bacterial strains were used for different purposes as protein overexpression or plasmid DNA amplification and are listed below. 


$\begin{array}{lll}\text { E.coli } \mathrm{K}-12 \mathrm{DH} 5 \alpha & \text { Amplification of plasmid DNA } & \text { Hannahan, } 1983 \\ \text { E.coli BL21 }(\mathrm{DE} 3) & \text { Protein overexpression } & \text { Stratagene }\end{array}$

\subsubsection{Preparation of competent E.colicells}

Competent E.coli cells are prepared by the method of Hannahan (1983):

\subsubsection{Transformation of competent E.colicells}

Transformation was performed by the following method:

1. Thaw on ice an aliquot of competent cells.

2. Add 100ng of plasmid DNA and mix using the pipette.

3. Incubate on ice for $30 \mathrm{~min}$ at least.

4. Heat shock the sample up to $1,5 \mathrm{~min}$ at $42^{\circ} \mathrm{C}$.

5. Incubate the sample for $5 \mathrm{~min}$ on ice.

6. Add $600 \mu \mathrm{l}$ of SOB or $800 \mu \mathrm{l}$ of $\mathrm{LB}$ and incubate for $45 \mathrm{~min}$ at $37^{\circ} \mathrm{C}$, shaking.

7. Centrifuge at $6000 \mathrm{rpm}$ for $2 \mathrm{~min}$.

8. Resuspend the pellet in $200 \mu \mathrm{l}$ of the supernatant and plate on an LB/agar plate with the appropriate antibiotic for selection.

9. Let dry and incubate the plate inverted at $37^{\circ} \mathrm{C}, \mathrm{ON}$.

- SOB: $20 \mathrm{~g} / 1$ bacto triptone, $5 \mathrm{~g} / 1$ yeast extract and $0,5 \mathrm{~g} / 1 \mathrm{NaCl}$. Adjust $\mathrm{pH}$ at 7 and autoclave. Add $10 \mathrm{ml}$ of sterile $2 \mathrm{M} \mathrm{MgCl}_{2}$.

- LB agar: $10 \mathrm{~g} / 1$ bacto triptone, $5 \mathrm{~g} / 1$ yeast extract, $10 \mathrm{~g} / 1 \mathrm{NaCl}$ and $15 \mathrm{~g} / 1$ agar. Adjust $\mathrm{pH}$ at 7 with $\mathrm{NaOH}$.

\subsubsection{Vectors and Oligonucleotides}

Various vectors were used for the subcloning of PCR products, overexpression of proteins and the construction of fusion proteins with reporter function as GFP.

Vector Utility Reference or source

$\begin{array}{lll}\text { pGEM-T-easy } & \text { Cloning of PCR products } & \text { Promega } \\ \text { pET-28 a,b,c } & \text { Overexpression of proteins } & \text { Novagen } \\ \text { pBluescript } & \text { Transcritption in vitro } & \text { Stratagene } \\ \text { pCK GFP S65C } & \begin{array}{l}\text { TM20:GFP fusion protein for } \\ \text { cell transfection }\end{array} & \text { (Reichel } \text { et al. 1996) }\end{array}$


For a variety of constructions the DNA amplification and modification by PCR techniques was necessary. The oligonucleotides used in this work for this purpose are listed below.

RT-PCR and cloning of AtTM4: 5' ATCATtgtCGACATGCCTAGTGAAgCTATg 3' 5' TTGAATGTCGACTGGCTCCTGAGATGAGCT 3'

Fusion protein TM20:GFP: $\quad$ 5' TCATACACGCATGCCCATGGCCTAATA 3'

5' GAATTCTCTCCCTCCGGATTTCTTGAT 3'

Fusion protein pET-LoopTM20: 5' CGTCGACCATGGGCAGAAAGAG 3'

5' GGGACTTCTCGAGATGTCAATCAG 3'

\subsection{Commercial kits}

For the use of commercially available kits, if not indicated specially, the manual delivered by the manufacturer was followed. Modifications of protocol steps will be mentioned.

\subsection{Analysis of Nucleic acids}

\subsubsection{Extraction and purification of DNA from E.coli (Minipreps and Midipreps)}

The preparation of plasmid DNA from E.coli was performed in two distinct manners, depending on the desired amount of DNA. For small scale extractions (Minipreps) the method of alkaline lysis was applied (Birnboim and Dolly 1979; Sambrook et al. 1989). For large scale preparations of guaranteed high purity (Midipreps) were performed.

\section{Miniprep:}

1. One bacterial colony is inoculated in $3 \mathrm{ml}$ of $\mathrm{LB}$ medium with the appropriate antibiotic and is left to incubate at $37^{\circ} \mathrm{C}$ overnight. This volume of bacterial culture is used for a mini preparation of DNA. 


\section{Material and Methods}

2. Centrifuge $3 \mathrm{ml}$ of culture at $13000 \mathrm{rpm}$ for $5 \mathrm{~min}$ at RT.

3. Resuspend the bacterial pellet in $150 \mu$ l of solution I.

4. Add $300 \mu \mathrm{l}$ of solution II.

5. Invert the tube gently several times and let on ice for $5 \mathrm{~min}$.

6. Add $225 \mu \mathrm{l}$ of solution III and mix by vortex.

7. Centrifuge at $13000 \mathrm{rpm}$ for $5 \mathrm{~min}$ at RT.

8. To the supernatant add $675 \mu \mathrm{l}$ of phenol $/ \mathrm{CH}_{3} \mathrm{Cl} / \mathrm{IsoAA}(25: 24: 1)$

and mix by vortex.

9. Centrifuge at $13000 \mathrm{rpm}$ for $10 \mathrm{~min}$ at RT.

10. To the aqueous phase add $675 \mu$ of $\mathrm{CH}_{3} \mathrm{Cl}$ and mix by vortex.

11. Centrifuge at $13000 \mathrm{rpm}$ for $5 \mathrm{~min}$ at RT.

12. To the supernatant add $675 \mu \mathrm{l}$ of isopropanol.

13. Centrifuge at $13000 \mathrm{rpm}$ for $15 \mathrm{~min}$ at $4^{\circ} \mathrm{C}$.

14. Wash the pellet with $200 \mu$ l ethanol $70 \%$.

15. Centrifuge at $13000 \mathrm{rpm}$ for $5 \mathrm{~min}$ at RT.

16. Resuspend the pellet in $40 \mu \mathrm{l}$ of $\mathrm{H}_{2} \mathrm{O}$ with RNase $100 \mu \mathrm{g} / \mathrm{ml}$.

- LB medium: $10 \mathrm{~g} / 1$ bactotriptone, $5 \mathrm{~g} / 1$ yeast extract, $10 \mathrm{~g} / 1 \mathrm{NaCl}$. Adjust $\mathrm{pH}$ at 7 with $\mathrm{NaOH}$.

- $\quad$ Solution I: $\quad 50 \mathrm{mM}$ glucose, $25 \mathrm{mM}$ Tris- $\mathrm{HCl} \mathrm{pH} 8,10 \mathrm{mM}$ EDTA.

- Solution II: $0,2 \mathrm{~N} \mathrm{NaOH}, 1 \%$ SDS.

- Solution III: $3 \mathrm{M}$ potassium acetate $\mathrm{pH} 4,8$.

\section{Midiprep:}

For Midi preparations the commercial plasmid extraction kit from Qiagen were used. The system of this kit is based in the alkaline lysis and the use of ionic exchange columns, which provide a high yield of, purified, DNase free DNA. Outgoing from $50 \mathrm{ml}$ bacterial culture $80-120 \mu \mathrm{g}$ DNA can be purified in the case of high copy plasmids as pBluescript.

\subsubsection{Extraction and purification of RNA from plants}

Two different methods were used to obtain RNA from plant material. First the RNA extraction based on washing steps with phenol/chloroform, which represents a modification of the protocol described by Logeman et al (1987). This protocol was used in the case if large amounts of RNA were necessary. Second, plant RNA was obtained using a kit for rapid plant RNA extraction (RNAeasy Plant Kit ${ }^{\mathrm{TM}}$, Qiagen) providing pure RNA. During all the handling with RNA it is obligatory to wear laboratory gloves to avoid RNA degradation originated from the RNases originated from the skin. All reactives used should be RNase free. 


\subsubsection{Phenol/Chloroform extraction of plant RNA}

Total RNA is extracted using the following method:

1. Prepare an RNase free morter and pestle by setting these at $200^{\circ} \mathrm{C}$ for 4 hours.

2. Grinding plant material with the morter and pestle using liquid nitrogen until the material is converted into a fine powder.

3. Aliquot the powdered material to RNase free Eppendorff tubes and add $400 \mu 1$ of Z6 buffer to the powder. Homogenize by inverting the tube several times.

4. Add an equal volume of phenol $/ \mathrm{CH}_{3} \mathrm{Cl} / \mathrm{IsoAA}$ (25:24:1), to each one.

5. Mix samples for $10 \mathrm{~min}$.

6. Centrifuge at $13000 \mathrm{rpm}$ for $5 \mathrm{~min}$ at. Recover the aqueous phase.

7. Add an equal volume of $\mathrm{CH}_{3} \mathrm{Cl}$ and mix by vortex.

8. Centrifuge at $13000 \mathrm{rpm}$ for $5 \mathrm{~min}$.

9. To each supernatant add 0,1 volumes acetic acid $1 \mathrm{M}$ and mix by vortex.

10. Add 2,5 volumes of ethanol $100 \%$ and mix by vortex.

11. Let the tubes at $-20^{\circ} \mathrm{C}$ for at least 1 hour.

12. Centrifuge at $13000 \mathrm{rpm}$ for $15 \mathrm{~min}$.

13. To each pellet add $1 \mathrm{ml}$ of $3 \mathrm{M}$ sodium acetate $\mathrm{pH} 5,2$ and vortex the samples briefly.

14. Centrifuge at $13000 \mathrm{rpm}$ for $20 \mathrm{~min}$.

15. Wash each pellet with $1 \mathrm{ml}$ of ethanol $70 \%$.

16. Centrifuge at $13000 \mathrm{rpm}$ for $10 \mathrm{~min}$.

17. Let the pellets to dry for $20 \mathrm{~min}$ at RT. Resuspend the pellets in a suitable volume of DEPC treated water.

18. Perform a DNase treatment as follows: let at $37^{\circ} \mathrm{C}$ the reaction composed by $88 \mu \mathrm{RNA}$ sample, $10 \mu \mathrm{l}$ of $10 x$ DNase buffer and $2 \mu \mathrm{l}$ of DNase/RNase free, for $30 \mathrm{~min}$.

19. Repeat steps 4 to 17 omitting step 13 (scale down all reagent volumes). Resuspend in $200 \mu l$ of DEPC treated water and store at $-70^{\circ} \mathrm{C}$.

- Buffer Z6: $191 \mathrm{~g}$ of $8 \mathrm{M}$ guanidine- $\mathrm{HCl}, 10 \mathrm{ml}$ of $0,5 \mathrm{M}$ EDTA, $\mathrm{H}_{2} \mathrm{O}$ up to $250 \mathrm{ml}$.

- Sodium acetate $3 \mathrm{M}$ pH 5,2: 24,6g sodium acetate, $100 \mathrm{ml}$ DEPC treated $\mathrm{H}_{2} \mathrm{O}$, autoclave.

- Acetic acid 1M: 5,7ml glacial acetic acid, 94,3ml DEPC treated $\mathrm{H}_{2} \mathrm{O}$.

- DEPC treated $\mathrm{H}_{2} \mathrm{O}: 500 \mu \mathrm{l}$ DEPC in $5 \mathrm{ml}$ ethanol $100 \%, 494,5 \mathrm{ml} \mathrm{H}_{2} \mathrm{O}$, autoclave twice.

- 10x DNase buffer: 50mM Tris- $\mathrm{HCl} \mathrm{pH} 7,5,10 \mathrm{mM} \mathrm{MgCl}_{2}$, 1mM DTT, RNasin 40 units.

\subsubsection{RNA extraction with the RNA easy plant kit}

Total RNA was extracted following the manufacturers protocol. The extraction method based on centrifugation columns is fast and yields smaller amounts of purified RNA.

\subsubsection{DNA digestion with restriction enzymes and ligation reactions}

For all digestions the DNA concentration is adjusted at $0,5 \mu \mathrm{g} / \mu \mathrm{l}$. One unit of enzyme is used for every $\mu \mathrm{g}$ of plasmid DNA in the appropriate digestion buffer and the reaction is left to incubate at $37^{\circ} \mathrm{C}$ for $1-3$ hours. The concentration of glycerol 


\section{Material and Methods}

should not overpass the $10 \%$ of the final reaction volume in all cases. The different restriction enzymes were acquired from Promega or Boehringer Mannheim.

Ligations are performed calculating the amounts of the insert and vector (in ng) according to the following equation (Maniatis et al., 1989):

"X" $\mathrm{X}^{\prime}=\operatorname{Insert}(\mathrm{ng})=$ Plasmid (ng) x MW of insert (in kb) / MW of plasmid (in kb)

This gives the 1 to 1 ratio of insert to vector quantity. For all ligations, a 3 to 1 ratio of insert to vector is used for each reaction. For 100ng of vector $3 x^{\prime \prime} X^{\prime \prime}$ ng of insert are used with 0,1 Weiss units of T4 ligase (Promega) and $1 \mu 1$ of 10x T4 ligase buffer (Promega) in a final volume of $10 \mu \mathrm{l}$. Incubation is performed at $16^{\circ} \mathrm{C}$ from $1 \mathrm{~h}$ to overnight.

\subsubsection{Dephosphorylation reaction with Alkaline Phosphatase (AP)}

To avoid the religation of a previously digested vector during the ligation process it is recommended to dephosphorylate the ends of the vector, since the T4 ligase enzyme requires a phosphorylated 5' end (sticky or blunt end) for the ligation process.

Dephosphorylation with alkaline phosphatase:

1. Mix approximately $60 \mathrm{ng}$ of DNA with $2 \mu 1$ reaction buffer and $1 \mathrm{U}$ alkaline phosphatase (Promega) in a microfuge tube. Add water to a final volume of $20 \mu 1$.

2. Perform reaction during $30 \mathrm{~min}$ at $37^{\circ} \mathrm{C}$.

3. Add $200 \mu 1 \mathrm{H}_{2} \mathrm{O}$.

4. Add the equal volume of phenol $/ \mathrm{CH}_{3} \mathrm{Cl} / \mathrm{IsoAA}(25: 24: 1)$

5. Centrifuge at $13000 \mathrm{rpm}$ for $5 \mathrm{~min}$.

6. Add $1 / 10 \mathrm{Vol} 3 \mathrm{M}$ sodium acetate $\mathrm{pH} 5,2$ and vortex.

7. Add $2,5 \mathrm{Vol} \mathrm{EtOH} 100 \%$ and vortex

8. Centrifuge at $13000 \mathrm{rpm}$ for $15 \mathrm{~min}$.

9. Discard supernatant and add $500 \mu \mathrm{LtOH} 70 \%$ to the pellet.

10. Centrifuge at $13000 \mathrm{rpm}$ for $10 \mathrm{~min}$.

11. Discard supernatant and resuspend the pellet in $15 \mu 1 \mathrm{TE}$ o $\mathrm{H}_{2} \mathrm{O}$.

AP reaction buffer: Promega

TE: 


\subsubsection{Generation of blunt ends by Klenow fragment reaction}

Some cloning strategies required the necessity to generate blunt end, even if the enzymatic digestion was performed with a restriction enzyme producing sticky ends. The Klenow fragment adds the lacking residues to the $5^{\prime}$ and $3^{\prime}$ end.

Blunt end generation by Klenow reaction:

1. Mix approximately 60ng DNA with $1,2 \mu 1$ Klenow buffer and $1 \mathrm{U}$ Klenow fragment (Boehringer Mannheim). Add $\mathrm{H}_{2} \mathrm{O}$ to a final volume of $11 \mu 1$.

2. Perform reaction for $5 \mathrm{~min}$ at $37^{\circ} \mathrm{C}$.

3. Add $1 \mu 1 \mathrm{dNTPs}$.

4. Incubate reaction for $10 \mathrm{~min}$ at $37^{\circ} \mathrm{C}$.

5. Inactivate the enzyme activity $5 \mathrm{~min}$ at $65^{\circ} \mathrm{C}$.

6. Depending on the following steps it would be necessary to clean the sample from enzyme and buffer by phenol $/ \mathrm{CH}_{3} \mathrm{Cl}$ / IsoAA (25:24:1), as described before (see step417 section 2.3.2.1).

Klenow reaction buffer: $100 \mathrm{mM}$ Tris $\mathrm{pH} 8.0,50 \mathrm{mM} \mathrm{MgCl}_{2}$ dNTP stock: $2 \mathrm{mM}$ each nucleotide

\subsubsection{Gel electrophoresis}

\subsubsection{Agarose gel electrophoresis of DNA}

DNA fragments were separated electrophoretically in an agarose gel of such percentage in agarose, according to the length of the DNA band to be visualised. A $1 \%$ agarose gel is prepared by melting in a microwave oven, $1 \mathrm{~g}$ of agarose in $100 \mathrm{ml}$ of $1 \mathrm{x}$ TAE buffer. The solution is left to cool down to $55^{\circ} \mathrm{C}$ and $\mathrm{EtBr}$ is added to a final concentration of $0,5 \mu \mathrm{g} / \mathrm{ml}$. The DNA samples are mixed with the appropriate volume of 10x loading buffer before loading these in the solidified gel. Running of the samples is performed with $1 \times$ TAE buffer under a suitable voltage (40-90V) for the time needed. DNA migrates towards the positive electrode.

- 10x Loading buffer: 0,25\% bromophenol blue, 0,25\% xylene cyanol, 50\% glycerol in $1 \times$ TE.

- 50x TAE buffer: 2M Tris-acetate $\mathrm{pH}$ 8,1, 0,1M EDTA.

\subsubsection{RNA Agarose gel electrophoresis under denaturating conditions}

RNA electrophoresis is performed in denaturing gels of $1 \%$ in agarose. The gel is prepared by mixing a solution of $10 \mathrm{ml}$ of $10 x$ MEN buffer with $18 \mathrm{ml}$ aldehyd $37 \%$, 


\section{Material and Methods}

with a second solution prepared by melting $1 \mathrm{~g}$ of agarose in $72 \mathrm{ml}$ of $\mathrm{H}_{2} \mathrm{O}$ and left to cool down to $60^{\circ} \mathrm{C}$. The desired amount of RNA sample is vacuum dried and resuspended in RNA loading buffer. Before the sample is loaded to the gel it is precisely to denaturate the RNA at $65^{\circ} \mathrm{C}$ for $5 \mathrm{~min}$ (Transfer the samples directly from the heat block to the gel). Electrophoresis is performed with 1x MEN buffer at a suitable voltage (50-90V) for the time needed. The whole process has to be performed in an extraction chamber due to the toxicity of formaldehyde.

- 10x Loading buffer: MEN 1x, formaldehyde 6,5\%, BrET 0,1 $\mu \mathrm{g} / \mathrm{ml}$, Bromphenol Blue (or DNA loading buffer) $0,6 x$.

- 10x MEN buffer: 200mM MOPS pH 7, 50mM sodium acetate, 10mM EDTA.

\subsubsection{Purification of DNA bands from agarose gels}

Purification of DNA bands from agarose gels was made by use of the "GFX" PCR DNA and Gel Band Purification Kit" (Amersham Pharmacia Biotech) following the protocol provided:

1. Weight an eppendorf tube.

2. Excise the DNA band to be purified from the gel and transfer it to the tube.

3. Weight the tube containing the agarose slice.

4. For every $10 \mathrm{mg}$ of gel weight add $10 \mu \mathrm{l}$ of Capture buffer.

5. Let the tube at $60^{\circ} \mathrm{C}$ until the gel slice dissolves completely.

6. Transfer the sample into a GFX purification column and place it inside an eppendorf tube.

7. Centrifuge at 13000rpm for 30sec at RT.

8. Discard the liquid from the eppendorf tube and place the column in it once again.

9. Add $500 \mu$ l of Wash buffer to the column and centrifuge at 13000rpm for 30sec at RT.

10. Discard the liquid and centrifuge once more.

11. Place the column into a new eppendorf tube.

12. Add $30 \mu$ l of $\mathrm{H}_{2} \mathrm{O}$ to the column and let for $1 \mathrm{~min}$ at RT.

13. Centrifuge at $13000 \mathrm{rpm}$ for $1 \mathrm{~min}$ at RT.

14. Collect the DNA elute at the eppendorf tube and store at $-20^{\circ} \mathrm{C}$ until further use.

- Capture buffer: Amersham Pharmacia Biotech.

- Wash buffer: $10 \mathrm{mM}$ Tris- $\mathrm{HCl}$ pH 8, 1mM EDTA pH 8, 80\% ethanol.

\subsection{7 in vitro transcription of RNA}

The in vitro RNA transcriptions were performed following the standard transcription protocol provided by Promega with slight modifications considering the length of the DNA template to be transcribed. 
1. Add the following components at room temperature in the order listed.

$\begin{array}{ll}\text { Transcription optimized buffer 5x } & 4 \mu 1 \\ \text { DTT 100mM } & 2 \mu 1 \\ \text { RNAsin Ribonuclease Inhibitor 20U } & 1 \mu 1 \\ \text { dNTPs 2.5mM each } & 8 \mu 1 \\ \text { linearized template DNA 0.2-1.0mg } & 2 \mu 1 \\ \text { T3 RNA Polymerase (15-20U / } \mu \mathrm{l}) & 1 \mu 1 \\ \mathrm{H}_{2} \mathrm{O} & \text { to a final volume of } 20 \mu 1\end{array}$

2. Incubate for $60 \mathrm{~min}$ at $37-40^{\circ} \mathrm{C}$

3. Treatment with DNase I RNase free to remove template DNA (see step18 section 2.3.2.1)

4. Wash steps with Phenol/Chloroform (see step 4-17 section 2.3.2.1)

Transcription optimized buffer 5x: (Promega)

T3 RNA Polymerase : (Promega)

\subsubsection{Poly Chain Reaction (PCR)}

The Polymerase Chain Reaction makes possible the synthesis of specific DNA sequences using two oligonucleotides complement to the two DNA strands. The reaction requires a Thermocycler, which controls the exact temperature for each of the different steps that characterize the PCR: melting, annealing and amplification. The amplification reaction is carried out by the special heat resistant Taq-polymerase. This technique provides a high variety of application possibilities, outgoing from different types of templates, as DNA, RNA or cDNA. Thus, the PCR can be used to confirm the presence of a DNA fragment in a plasmid, the expression of certain gene (RT-PCR) or can prepare DNA fragments for clonation. The reaction provides several factors, beside of the temperature, to adjust the reaction to optimal conditions, as the concentration of magnesium chloride.

Standardized PCR protocol:

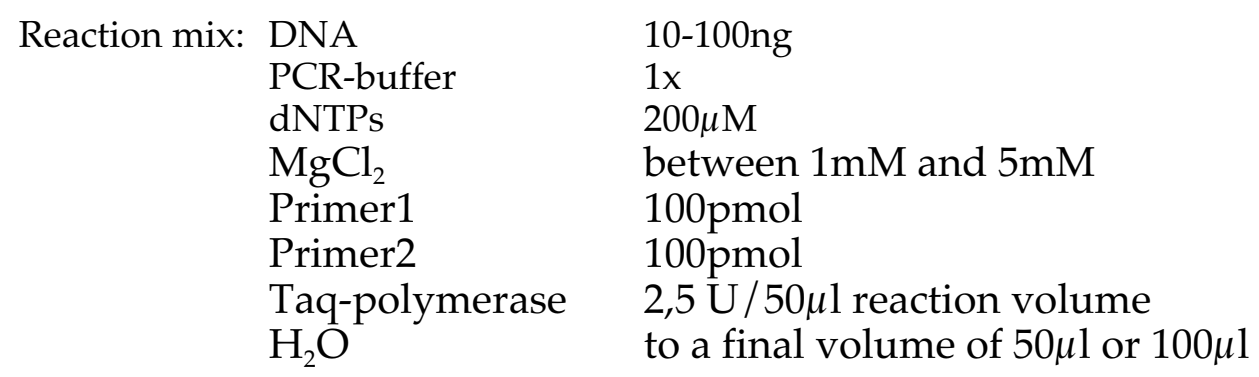


Cycle parameters:

$\begin{array}{lll}94^{\circ} \mathrm{C} & 2 \mathrm{~min} & 1 \mathrm{cycle} \\ 94^{\circ} \mathrm{C} & 1 \mathrm{~min} & 30 \mathrm{cycles} \\ 60^{\circ} \mathrm{C} & 1 \mathrm{~min} & \begin{array}{l}\text { (temperature depends on the Tm on the applied Primers) } \\ \text { (time depends on the length of the fragment; } 1 \mathrm{~kb} / \mathrm{min} \text { ) }\end{array} \\ 72^{\circ} \mathrm{C} & \mathrm{X} \mathrm{min} & 1 \mathrm{cycle}\end{array}$

PCR buffer 10x: 500mM KCL, 200mM Tris-HCl pH 8,6, 1\% Triton x-100

\subsubsection{RT-PCR}

The RT-PCR technique is based in the retranscription of RNA, free of DNA, to cDNA by the enzyme Retranscriptase employing an oligonucleotide poly-dT, which will bind to the polyAcoil of the RNA. The cDNA originated from RNA is used as template for subsequently performed PCR.

Retranscription reaction:

1. Mix in a microfuge tube: RNA (DNA free)

RNAsin $(24,8 \mathrm{U} / \mu 1)$

$1 \mu \mathrm{g}-5 \mu \mathrm{g}$

Oligo-dT

$\mathrm{H}_{2} \mathrm{O}$

$2 \mu 1$

200pmol

till a volume of $11,5 \mu 1$

2. Denaturate the RNA for $5 \mathrm{~min}$ at $65^{\circ} \mathrm{C}$ and transfer the mix immediately to ice.

3. Add the following:

$\begin{array}{ll}\text { RT buffer } 5 \mathrm{x} & 4 \mu 1 \\ \text { DTT }(100 \mathrm{mM}) & 2 \mu 1 \\ \text { dNTPs } 10 \mathrm{mM} \text { each } & 1 \mu 1 \\ \text { RNAsin }(24,8 \mathrm{U} / \mu \mathrm{l}) & 0,5 \mu 1 \\ \text { BSA }(1 \mathrm{mg} / \mathrm{ml}) & 1 \mu 1 \\ \text { M-MuLV-RT }(200 \mathrm{U} / \mu \mathrm{l}) & 1 \mu 1\end{array}$

4. Incubate for 1 hour at $42^{\circ} \mathrm{C}$.

5. Add $80 \mu 1$ of $\mathrm{H}_{2} \mathrm{O}$ and store at $-70^{\circ} \mathrm{C}$.

RT-buffer 5x: 100mM Tris- $\mathrm{HCl}$ pH8.4, 250mM KCl, 15mM MgCl 2 .

Oligo-dT: $5^{\prime}$-gct agg cca ctgtgg $\mathrm{ccT}_{15} \mathbf{-}^{\prime}$.

M-MuLV-RT 5x buffer: (Promega)

M-MuLV-RT : (Promega)

RT-PCR conditions:

$\begin{array}{lll}\text { 1. Mix : } & \text { cDNA } & \text { 100ng-250ng } \\ & \text { PCR-buffer } & 1 \mathrm{x} \\ & \text { dNTPs } & 200 \mu \mathrm{M} \\ & \mathrm{MgCl}_{2} & 2.5 \mathrm{mM}\end{array}$




\author{
Primer1 100pmol \\ Primer2 100pmol \\ Taq-polymerase $\quad 2.5 \mathrm{U} / 50 \mu 1$ reaction volume \\ $\mathrm{H}_{2} \mathrm{O} \quad$ till the desired reaction volume $(50 \mu 1$ or $100 \mu 1)$
}

2. PCR reaction with the following conditions:

$\begin{array}{lll}94^{\circ} \mathrm{C} & 2 \mathrm{~min} & 1 \text { cycle } \\ 94^{\circ} \mathrm{C} & 1 \mathrm{~min} & 35 \mathrm{cycles} \\ 60^{\circ} \mathrm{C} & 1 \mathrm{~min} & \begin{array}{l}\text { (temperature depends on the Tm of the applied Primers) } \\ \text { (time depends on the length of the fragment; } 1 \mathrm{~kb} / \mathrm{min} \text { ) }\end{array} \\ 72^{\circ} \mathrm{C} & \mathrm{X} \mathrm{min} & 1 \text { cycle }\end{array}$

\title{
2.3.9 Sequencing of DNA
}

Sequencing of DNA clones was performed automatically using oligonucleotides labelled with fluorescence (Automated Laser Fluorescence) and using the Applied Biosystems sequencing system (Pharmacia). The Sequencing process was performed by the Servei de Seqüenciació Automatica of the IBMB (Institut de Biologia Molecular Barcelona, CSIC, Barcelona).

\subsection{Protein analysis}

\subsubsection{Protein Extractions from plants}

\subsubsection{Extraction of total protein} protocol:

Extraction of total proteins from plant tissues is performed with the following

1. Grind 2-3mg of plant tissue with a morter and pestle, using liquid $\mathrm{N}_{2}$.

2. Transfer enough ground tissue to a microfuge tube up to the indication of $500 \mu$.

3. Add $300 \mu l$ of extraction buffer and vortex for $1 \mathrm{~min}$.

4. Centrifuge at $4000 \mathrm{rpm}$ for $10 \mathrm{~min}$ at $4^{\circ} \mathrm{C}$.

5. Transfer the supernatant to a new microfuge tube and centrifuge at $13000 \mathrm{rpm}$ for $10 \mathrm{~min}$ at $4^{\circ} \mathrm{C}$.

6. Transfer the supernatant to a new microfuge tube and proceed with quantification of the sample (see section 2.4.1.4).

7. Guard at $-20^{\circ} \mathrm{C}$.

- Extraction buffer: 50mM Tris-HCl pH 8, $10 \mathrm{mM} \mathrm{NaCl}, 1 \%$ SDS, $5 \%$ ßmercaptoethanol. Before use, add the following protease inhibitors: leupeptin 0,5ug $/ \mathrm{ml}$, pepsatin $1 \mu \mathrm{g} / \mathrm{ml}$, aprotinin $10 \mu \mathrm{g} / \mathrm{ml}$, E64 $1 \mu \mathrm{g} / \mathrm{ml}$ (in 50\% ethanol), PMSF $0,1 \mathrm{mM}$ (200mM stock in isopropanol). 


\section{Material and Methods}

\subsubsection{Extraction and separation of membrane fractions by differential Ultracentrifugation}

To separate the fraction of soluble proteins from the proteins associated to the membrane (integral or membrane bound) the following protocol is performed.

1. Homogenize plant tissue with a pestle in a ice-precooled morter adding $1 \mathrm{ml}$ of microsomal extraction buffer (Do not use liquid $\mathrm{N}_{2}$ )

2. Wash pestle and morter with $1 \mathrm{ml}$ microsomal extraction buffer.

3. Aliquot the sample to microfuge tubes and vortex. If it is necessary add microsomal extraction buffer to dilute the sample. The sample has to be liquid not viscous.

4. Centrifuge at $4000 \mathrm{rpm}$ for $10 \mathrm{~min}$ at $4^{\circ} \mathrm{C}$.

5. Transfer supernatant to a new microfuge tube and centrifuge at $13000 \mathrm{rpm}$ for $10 \mathrm{~min}$ at $4^{\circ} \mathrm{C}$.

6. Transfer supernatant to a sample tube for ultracentrifugation.

7. Centrifuge at $>150000 \mathrm{~g}$ (32500rpm rotor Beckman SW60 Ti; 28000rpm Beckman SW40 $\mathrm{Ti}$; Ultracentrifuge Beckman Optima $\left.{ }^{\mathrm{TM}} \mathrm{LE}-80 \mathrm{~K}\right)$ for $1.5 \mathrm{~h}$ at $4^{\circ} \mathrm{C}$.

8. Recover aliquot of the supernatant and precipitate proteins with TCA or acetone (see section 2.4.1.3).

9. Resuspend pellet in PBS. If necessary use a glass homogenizer (Afora, Spain) and vortex.

10. Proceed with protein quantification of the sample (see section 2.4.1.4).

11. Store at $-80^{\circ} \mathrm{C}$.

Microsomal extraction buffer: $100 \mathrm{mM}$ Tris- $\mathrm{HCl} \mathrm{pH} 7.9,12 \%$ sucrose, $1 \mathrm{mM}$ EDTA, protease inhibitors: leupeptin $0,5 \mu \mathrm{g} / \mathrm{ml}$, pepsatin $1 \mu \mathrm{g} / \mathrm{ml}$, aprotinin $10 \mu \mathrm{g} / \mathrm{ml}$, E64 $1 \mu \mathrm{g} / \mathrm{ml}$ (in 50\% ethanol), PMSF 0,1mM (200mM stock in isopropanol).

\subsubsection{Protein precipitation with TCA and acetone}

As a method for protein concentration or to change the buffer solution protein precipitation following two protocols is employed.

Protein precipitation with acetone:

1. Add $80 \%$ precooled acetone $(100 \%)$ to the sample solution.

2. Centrifuge for $20 \mathrm{~min}$ at $4^{\circ} \mathrm{C}$

3. Discard the supernatant and let dry the pellet at room temperature.

4. Resuspend the pellet in a suitable buffer or directly in protein loading buffer (TMx2).

Protein precipitation with Trichloroacetic acid (TCA):

1. Add TCA to the sample to a final concentration of $15 \%$.

2. Let for $20 \mathrm{~min}$ on ice.

3. Centrifuge at $13000 \mathrm{rpm}$ for $10 \mathrm{~min}$ at $4^{\circ} \mathrm{C}$.

4. Discard the supernatant and add precooled acetone $\left(-20^{\circ} \mathrm{C}\right)$ and sonicate for $1 \mathrm{~min}$.

5. Centrifuge at $13000 \mathrm{rpm}$ for $20 \mathrm{~min}$ at $4^{\circ} \mathrm{C}$.

6. Discard supernatant and resuspend the pellet in a suitable buffer or directly in protein loading buffer (TMx2). 
Protein loading buffer TMx2: 20\% Glycerol, 4\% SDS, 0.125M Tris, 0.04\% Bromofenol blue, adjust to pH6.8. Add 10\% ß-mercaptomethanol directly before use.

\subsubsection{Protein quantification}

Quantification of proteins is performed using the method of Bradford (1976) following the standard protocol provided by the manufacturer of the Bradford reagent Bio-Rad:

1. A standard curve is made by the following way:

a. Prepare 6 standard samples of known concentration e.g. 0, 1, 2, 5, 10 and $15 \mu \mathrm{g} / \mu \mathrm{l}$ BSA.

b. To these volumes, add $\mathrm{H}_{2} \mathrm{O}$ up to $800 \mu \mathrm{l}$.

c. Add $200 \mu \mathrm{l}$ of Bradford reagent (Bio-Rad) and let for minimum $5 \mathrm{~min}$ at RT. The colour reaction will be stable for up to 1 hour.

d. Measure the optical density $\left(\mathrm{OD}_{595}\right)$.

e. Draw a graph of the $\mathrm{OD}_{595}$ values against the known concentration of each standard sample.

2. Proceed with measurement of the sample(s) concentration as for standard samples.

Another quick method for protein quantitation is by the absorbance of UV irradiation (Harlow and Lane 1988). At wavelength 280nm the absorbance maximum is due primarily the presence of tyrosine and tryptophan. The use of this method does not destroy the sample.

1. Read the absorbance versus a suitable control at $280 \mathrm{~nm}$.

2. A rough approximation for proteins is $A_{280}=1$ corresponds to $1 \mathrm{mg} / \mathrm{ml}$. For antibodies: IgG: $\mathrm{A}_{280}=1.35$ corresponds to $1 \mathrm{mg} / \mathrm{ml}$.

\subsubsection{Expression and purification of recombinant protein in E.coli using the pET system}

Overexpression of recombinant proteins was performed using the pET System (Novagen). Overexpression and purification of proteins was performed according to the following protocol provided by the manufacturer.

\subsubsection{Antibody generation in rabbit}

The animal of choice for the production of polyclonal antibodies was rabbit (Oryctolagus cuniculus) variety New Zealand White. These animals are easy to handle 


\section{Material and Methods}

and maintain under laboratory conditions and provide a higher amount of serum than do rats, hamsters or mice. The blood extraction were performed from the ear blood vessel which is characterized by easy and relatively painless access (Harlow and Lane 1988) utilizing the Vacutainer ${ }^{\circledR}$ Brand Systems Blood collection set (Beckton Dickinson, UK).

\subsubsection{Immunization}

The first immunization was performed, when the animal was two month old and had been habituated to the environment. Four days before a blood extraction were carried out to obtain serum preimmune. The 4 immunizations took place in intervals of 30-40 days to assure a sufficient response from the immunological system. From the second immunization on the antibody production was monitored by blood extraction 8-10 days after the immunization.

For the first immunization complete Freunds adjuvant or CFA (Sigma) were used to provoke a stronger immunological response. In the subsequent immunization steps the incomplete Freunds adjuvant or IFA (Sigma) were employed (Harlow and Lane 1988).

Antigen preparation for injection:

1. Dilute $\sim 100 \mu \mathrm{g}$ of antigen in $500 \mu 1-1000 \mu 1$ PBS.

2. Add 1 Vol Freunds adjuvant (CFA or IFA depending on the immunization step) and vortex well up to obtain a homogeneous emulsion.

3. Inject the sample subcutaneous in five different localisations.

\subsubsection{Antibody preparation, storage and purification}

The usage of the Vacutainer® Collection System facilitates the separation of the serum from the rest of undesired blood components. The antibodies raised against synthetic polypeptides were affinity purified employing the SulfoLink ${ }^{\mathrm{TM}}$ purification kit (Pierce).

\section{Antibody preparation and storage:}

1. Centrifuge the container tubes with the collected blood at $5000 \mathrm{rpm}$ for $15 \mathrm{~min}$ at $4^{\circ} \mathrm{C}$ (centrifuge B.Braun Sigma 2-15, Germany).

2. Recover the upper phase, which is the antibody containing serum and discard the rest.

3. Add $0.03 \%$ sodium azide $\left(\mathrm{NaN}_{3}\right)$ and aliquot the serum.

4. Store at $-80^{\circ} \mathrm{C}$. 


\section{Antibody purification:}

The antibodies derived from the injection of synthetic peptides were affinity purified against their corresponding peptides. For that purpose the SulfoLink ${ }^{\circledR}$ Coupling Gel (Pierce) was used to immobilize the antigens by disulphide bonds to the terminal cysteine. Passing the polyclonal serum through the column the antigen specific antibodies were retained in the column and eluted after several wash steps. This fast and easy method purifies and concentrates the antibody. $1 \mathrm{ml}$ of gel binds up to $1 \mathrm{mg}$ sulfhydryl-containing peptide.

The antibody purification was performed following the manual provided by the manufacturer of SulfoLink®Coupling Gel.

\subsubsection{Immunodetection and immunohistochemistry}

\subsubsection{Western blotting}

The technique of western blotting was used for protein detection by the use of antibodies, including the protein separation by gel electrophoresis, protein transfer from the gel to a membrane, immunolabeling of the membrane and visualization of the labelled proteins.

\subsection{SDS-PAGE}

For the separation of proteins by gel electrophoresis a 10 to $15 \%$ polyacrylamide gel is used The percentage of acrylamide/bisacrylamide depends on the molecular weight of the protein(s) to be studied. The proteins are firstly introduced in a short length (stacking) gel of low acrylamide/bisacrylamide percentage, normally 3\%, which allows a more efficient introduction to the separation (lower) gel (10-15\% acrylamide/bisacrylamide). The protein samples are prepared by mixing one volume of protein extract of known concentration with a volume of $2 x$ sample buffer. Electrophoresis is performed with electrophoresis buffer at $120 \mathrm{~V}$ until the bromophenol blue dye reaches the end of the gel.

- Lower gel of $15 \%$ in acrylamide/bisacrylamide: $2,5 \mathrm{ml}$ lower buffer, $3,75 \mathrm{ml}$ acrylamide/bisacrylamide (30:0,8) 40\%, 3,75 $\mathrm{ml} \mathrm{H}_{2} \mathrm{O}, 5 \mu 1$ TEMED, 40,4 $\mu 1$ APS 15\%. 


\section{Material and Methods}

- Stacking gel of $3 \%$ in acrylamide/bisacrylamide: $0,5 \mathrm{ml}$ upper buffer, $0,15 \mathrm{ml}$ acrylamide/bisacrylamide (30:0,8) 40\%, 1,3 $\mathrm{ml} \mathrm{H}_{2} \mathrm{O}, 2 \mu 1$ TEMED, 20 $\mu 1$ APS 15\%.

- Lower buffer: $1,5 \mathrm{M}$ Tris $\cdot \mathrm{HCl} \mathrm{pH} 8,8,10 \%$ SDS:

- Upper buffer: 0,5M Tris $\cdot \mathrm{HCl} \mathrm{pH} 6,5,0,4 \%$ SDS.

- $10 \mathrm{x}$ electrophoresis buffer: $0,25 \mathrm{M}$ Tris, $1,92 \mathrm{M}$ glycine, $1 \% \mathrm{SDS}, \mathrm{pH} 8,5$. 2x sample buffer: $125 \mathrm{mM}$ Tris $\cdot \mathrm{HCl}$ pH 6,8, 4\% SDS, $20 \%$ glycerol, $0,04 \%$ bromophenol blue.

\subsection{Electroblotting}

Electroblotting was performed using the Protein Transfer Tank System (BioRad). The electrophoretically separated proteins were transferred from the SDSPAGE gel to a PVDF membrane (Immobilon-P, Millipore) in an electric field. The Tank System is characterised by the total submergence of the Gel-membrane sandwich in the transfer buffer. The Transfer was carried out normally overnight at $4^{\circ} \mathrm{C}$ at a voltage of 20-35 $\mathrm{V}$ on a magnetic steerer for a better circulation of the transfer buffer. Due to the highly hydrophobic character of the PVDF-membranes a pretreatment with methanol before use was necessary.

Protein Transfer in the Tank System:

1. Equilibrate polyacrylamide gel in the transfer buffer for $10 \mathrm{~min}$ at RT.

2. Cut the PVDF membrane to a suitable size and wet in $100 \%$ methanol for $20 \mathrm{sec}$ and wash afterwards several times with PBS-T. Do not let dry.

3. Wet transfer sponges and two pieces of Transfer Blotting Paper (BioRad) with transfer buffer.

4. Mount the transfer sandwich in the following order: positive pole-sponge-blotting paper-PVDF membrane-gel-blotting paper-sponge-negative pole.

5. Fill the tank up with transfer buffer till gel and membrane are totally submerged.

6. Let transfer overnight at $4^{\circ} \mathrm{C}$ with a voltage of $20 \mathrm{~V}-35 \mathrm{~V}$.

7. Take out the gel and the membrane. Stain the gel with coomassie blue (see section 2.4.4.1.5) to control the correct transfer. Proceed with the antibody incubation

Transfer buffer: 25mM Tris-HCl pH8.3; 192mM Glycine; 0.1\% SDS; $10 \%$ methanol. PBS buffer: $1.6 \mathrm{mM} \mathrm{PO}_{4} \mathrm{H}_{2} \mathrm{Na} \cdot \mathrm{H}_{2} \mathrm{O}, 8.4 \mathrm{mM} \mathrm{PO}_{4} \mathrm{HNa}_{2} \cdot 2 \mathrm{H}_{2} \mathrm{O}, 150 \mathrm{mM} \mathrm{NaCl}, \mathrm{pH} 7.4$. PBS-T: 0.1\% Tween 20 in PBS.

\subsection{Immunodetection}

1. Block membrane with blocking solution for 1 hour at RT, shaking.

2. Wash three times for $5 \mathrm{~min}$ with PBS-T.

3. Incubate with the primary antibody for 1 hour to overnight. The applied antibody concentration was normally 1:100 in incubation solution.

4. Wash three times for $5 \mathrm{~min}$ with PBS-T. 
5. Incubate with the secondary antibody, $\alpha$-rabbit IgG conjugated to horseradish peroxidase at a concentration of $1 / 10000$ in incubation solution for $45 \mathrm{~min}$ at RT.

6. Wash three times for $5 \mathrm{~min}$ with PBS-T.

7. The detection assay with ECL has to be carried out in the dark chamber.

8. Cover the membrane with the revelation solution, prepared before by mixing equal parts of the two components.

9. Incubate for $1 \mathrm{~min}$

10. Cover the membrane with shannah wrap paper and place an autoradiography film (Kodak) above.

11. Expose for different time intervals depending on the strength of the signal.

12. Reveal autoradiography.

Blocking solution: 5\%skimmed milk in PBS-T.

Incubation solution: $1 \%$ skimmed milk in PBS-T.

Revelation solution: commercial ECL (Amersham pharmacia biotech).

\subsection{Gel coomassie blue staining}

1. Submerge the protein gel in coomassie blue and let shaking for 20min at RT.

2. Distain the gel for $30 \mathrm{~min}$ using a solution of $30 \%$ methanol, $10 \%$ acetic acid.

Proceed various times, changing the solution each time, until the protein bands can be visualized.

\subsubsection{Immunolocalization in plant tissue}

This technique were used to detect specifically proteins in plant tissue sections or in whole mount. The process includes the fixing of the fresh tissues, the embedding in a suitable material to perform the sectioning, the immunolabeling employing a specific antibody which normally will be recognized by a secondary antibody tagged with a fluorescent dye. In this work all immunolocalizations were carried out employing a biotin coupled secondary antibody. In a third incubation step fluorescence tagged streptavidin bound to biotin causing an amplification effect for the marked antibodies. The visualization is performed by Fluorescence light microscopy or by Laser confocal microscopy.

\subsection{Sample fixation}

The fixation of the fresh material is important to a) stop the enzyme activity which can lead to the degradation of the tissue; b) avoid artefacts due to diffusion processes of peptides and proteins; c) fortify the tissue for the subsequent treatments. 


\section{Material and Methods}

Fixation with EtOH-acetic-acetic acid (paraffin embedded samples)

1. Fix the fresh tissue for 1 hour in the fixing solution. If it is necessary apply vacuum for $30 \mathrm{~min}$ at RT for a better penetration of the fixative.

2. Change fixing solution and incubate for 1 week at $4^{\circ} \mathrm{C}$.

3. Remove fixing solution and incubate $30-60 \mathrm{~min}$ with $70 \% \mathrm{EtOH}$ at RT.

4. Change $70 \% \mathrm{EtOH}$ against fresh solution of $70 \% \mathrm{EtOH}$ and store at $4{ }^{\circ} \mathrm{C}$. The samples can be stored at $4^{\circ} \mathrm{C}$ for several months.

Fixing solution: EtOH-acetic-acetic acid glacial (80:3.5:5 v:v:v)

Fixation with $3.5 \%$ paraformaldehyde (whole mount)

1. Prepare $3.5 \%$ paraformaldehyde and dissolve in PBS (heat to $60^{\circ} \mathrm{C}$ on a magnetic steerer until the paraformaldehyde is totally dissolved; work beneath a extractor).

2. Cool down the solution temperature on ice and filter to a $0.45 \mu \mathrm{m}$ pore filter (Schleicher\&Schuell, Germany). Aliquot and store at $-20^{\circ} \mathrm{C}$.

3. Incubate in fixing solution for 1-2 hours at RT under vacuum for better fixative penetration.

4. Change the fixing solution and incubate overnight at $4^{\circ} \mathrm{C}$.

5. Change fixing solution against store solution and store at $4^{\circ} \mathrm{C}$.

Fixing solution: 3.5\% paraformaldehyde in PBS.

Storing solution: $0.1 \%$ paraformaldehyde in PBS.

\subsection{Embedding in paraffin}

The embedding process consists in the substitution of the water of the sample against a suitable inert media, in this case paraffin (Paraplast Embedding Media, Sigma).

1. The samples are stored in 70\% EtOH. Change 70\% EtOH against fresh solution of $70 \% \mathrm{EtOH}$ and incubate for 1 hour.

2. Dehydrate the samples incubating 30-60min in each step of the following series of alcohol concentrations: $80 \% \mathrm{EtOH}, 0.5 \%$ Fast Green in $90 \% \mathrm{EtOH}, 100 \% \mathrm{EtOH}, 100 \%$ $\mathrm{EtOH}, 25 \%$ Tert-Butanol (TBA) in EtOH, 50\% TBA in EtOH, 100\% TBA, 100\% TBA.

3. Change TBA for fresh TBA and add an equal volume of paraffin (Paraplast Embedding Media, Sigma), preheated to $60^{\circ} \mathrm{C}$.

4. Incubate at $60^{\mathrm{a}} \mathrm{C}$ for 12 hours.

5. Remove solution and add fresh paraffin $\left(60^{\mathrm{a}} \mathrm{C}\right)$. Incubate for 12 hours at $60^{\circ} \mathrm{C}$.

6. Remove solution and add fresh paraffin $\left(60^{\circ} \mathrm{C}\right)$. Incubate for 2 hours at $60^{\circ} \mathrm{C}$.

7. Transfer the paraffin with the samples to a suitable container (petri plate) and separate with a tooth stick the samples.

8. Let solidify the paraffin.

9. Store the plates with paraffin at $4^{\circ} \mathrm{C}$.

Fast green is used to stain the samples for a better visibility embedded in the white paraffin. 


\subsection{Sample sectioning}

For a comfortable handling during the sectioning process the embedded samples are mounted on paraffin supports of suitable dimensions.

1. Isolate the desired sample using a hot scalpel or razor blade and mount the sample in the correct orientation on the paraffin support block.

2. Let the paraffin solidify.

3. Cut the borders of the sample containing paraffin block with a scalpel to a pyramidal shape.

4. Section the sample using a microtome (Reichert-Jung, Supercut, Germany) into sections of $8 \mu \mathrm{m}$.

5. Transfer the sections to a sterile petri plate using a tooth stick or a preparation needle.

6. Mount the sample on microscope slides pre-treated with $50 \mu \mathrm{g} / \mathrm{ml}$ of poly-D-Lysine. It is recommendable cover the slides with $1-2 \mathrm{ml} \mathrm{H}_{2} \mathrm{O}$ to expand the paraffin sections totally.

7. Suck the water with filter paper.

8. Let the slides dry on heating plates at $42^{\circ} \mathrm{C}$ overnight.

9. Store slides with silica gel $\left(3-6 \mathrm{~mm}\right.$, Panreac) at $4^{\circ} \mathrm{C}$ or proceed directly with the immunodetection (following section).

Treatment of microscope slides: submerge slides in $50 \mu \mathrm{g} / \mathrm{ml}$ poly-D-lysine in Tris$\mathrm{HCl} \mathrm{pH} 8.0$ for $30 \mathrm{~min}$ and let dry.

\subsection{Immunodetection}

Protocol for the paraffin embedded sample sections:

1. Remove the paraffin from the samples mounted on slides by incubation 2 times in xylol for $10 \mathrm{~min}$ at RT.

2. Rehydrate in series of decreasing EtOH concentrations: $100 \% \mathrm{EtOH}, 100 \% \mathrm{EtOH}$, $90 \% \mathrm{EtOH}, 70 \% \mathrm{EtOH}, 50 \% \mathrm{EtOH}, 30 \% \mathrm{EtOH}, \mathrm{H}_{2} \mathrm{O}, \mathrm{H}_{2} \mathrm{O}$. Incubate $2 \mathrm{~min}$ in each step.

3. Wash for 5 min with $\mathrm{H}_{2} \mathrm{O}$.

4. Incubate with $0.5 \%$ Triton $\mathrm{x}-100$ for $10 \mathrm{~min}$ at $\mathrm{RT}$.

5. Wash 2 times with wash buffer for $10 \mathrm{~min}$ at RT.

6. Incubate with block buffer for 1hour at RT.

7. Wash 2 times with wash buffer for 10 min at RT.

8. Incubate with the primary antibody for 2 hours at RT or overnight at $4^{\circ} \mathrm{C}$. The applied concentrations are normally higher than in western blot assays. $150 \mu 1 /$ slide is enough solution to cover the whole slide.

9. Wash 2 times with wash buffer for 10min at RT.

10. Incubate with the biotin conjugated secondary antibody for 1hour at RT. The applied concentration corresponds to the concentration recommended by the manufacturer $(1: 500)$.

11. Wash 2 times with wash buffer for $10 \mathrm{~min}$ RT.

12. Incubate with streptadivine tagged with Rhodamine Red or Oregon green 488 for 1 hour in the dark at Rt. The applied concentration corresponds to the concentration recommended by the manufacturer (1:100).

13. Wash 2 times with wash buffer for $10 \mathrm{~min}$ at RT.

14. Remove with caution excess of solution and mount with slides with Mowiol.

15. Store at $-20^{\circ} \mathrm{C}$ or proceed with the visualization by microscopy. 


\section{Material and Methods}

Triton x-100: 0.5\% Triton x-100 in wash buffer.

Wash buffer: PBS, $0.1 \%$ Tween 20, 20mM Glycine, $1 \%$ BSA.

Block buffer: 4-6mg/ml goat serum (Jackson Immunoresearch, USA) (host animal of secondary antibody) in wash buffer.

Antibody solutions: corresponding antibody in wash buffer.

Secondary antibody: (Jackson Immunoresearch, USA)

Streptavidin Rhodamine Red and Oregon green 488: (Jackson Immunoresearch, USA)

Mowiol: Mount media (Calbiochem)

All incubation steps were carried out in a humid chamber to avoid the sample to dry out. During the incubation the slide were covered with coverslips to provide an equal distribution of the solution all over the slide.

Protocol for whole mount immunodetection:

1. Wash the fixed sample 3 times for $10 \mathrm{~min}$ with PBS at RT.

2. Incubate for $30 \mathrm{~min}$ at RT with $1 \%$ Cellulysin Cellulase (Calbiochem, LaJolla,USA) and 1\% pectolyase Y-23 (Kikkoman, Tokyo, Japan) in PBS for cell wall digestion.

3. Wash 3 times for $5 \mathrm{~min}$ with PBS at RT.

4. Incubate with $0.1 \%$ Triton $x-100$ in PBS for $5-10 \mathrm{~min}$ at RT. This step is performed to permeabilize the cells and to guarantee a good penetration of the antibodies.

5. Wash 3 times for $5 \mathrm{~min}$ with PBS at RT.

6. Incubate with block buffer for 1hour at RT.

7. Wash 3 times for $5 \mathrm{~min}$ with PBS at RT.

8. Incubate with the primary antibody for 2hours at RT.

9. Wash 3 times for 5min with PBS at RT.

10. Incubate with the secondary, biotin-conjugated antibody for 1hour at RT.

11. Wash 3 times for $5 \mathrm{~min}$ with PBS at RT.

12. Incubate with streptadivine tagged with Rhodamine Red or Oregon green 488 for 1hour in the dark at Rt. The applied concentration corresponds to the concentration recommended by the manufacturer (1:100).

13. Wash 3 times for $5 \mathrm{~min}$ with PBS at RT.

14. Transfer the samples to microscopy slides and mount with Mowiol.

15. Visualize with confocal microscopy. Store at $4^{\circ} \mathrm{C}$.

Block buffer: 4-6mg/ml goat serum (Jackson Immunoresearch, USA) (host animal of secondary antibody) in PBS + 0.5\% BSA.

Antibody solution: suitable antibody concentration in PBS $+0.5 \%$ BSA.

All the steps of this protocol were carried out in $1.5 \mathrm{ml}$ microfuge tubes. At the beginning of every incubation vacuum were applied for $1 \mathrm{~min}$ for better penetration of the antibodies into the cells.

\subsection{Visualization}

\subsection{Light fluorescence microscopy}

For light fluorescence microscopy the Axiophot microscope (Zeiss,Germany) were used. The microscope is equipped with two cameras for the photographic documentation, UV lamp and filters with the following wavelength range: 


$\begin{array}{llll}\text { I } & 450-490 \mathrm{~nm} & \text { FT 510 } & \text { LP 520 } \\ \text { II } & \text { 395-440nm } & \text { FT 460 } & \text { LP 470 } \\ \text { III } & \text { BP 546nm } & \text { FT 580 } & \text { LP 590 }\end{array}$

\subsection{Confocal laser scanning microscopy}

A Leica TCS SP confocal laser scanning microscope (Heidelberg, Germany) fitted to/with spectrophotometers for emission band wavelength selection was used with two lasers, i.e., an argon ion laser emitting at $488 \mathrm{~nm}$ and an HeNe laser emitting at $543 \mathrm{~nm}$ to excite Oregon-green 488 and Rhodamin-Red, respectively. During scanning, we used a triple-dichroic beam splitter (TD 488/543/633). For visualization of Oregon-green 488, the emission window was set at $495-535 \mathrm{~nm}$. For visualization of Rhodamin-Red and Cy3, the emission window was set at 548-600 $\mathrm{nm}$. Confocal image stacks were combined as $\mathrm{x}-\mathrm{y}$ projection images.

\subsection{Transfection of onion epidermis cells}

Transfection were performed according to a modification of the protocol of Klein et al (1987):

Preparation of gold particles

1. Weight $60 \mathrm{mg}$ of gold particles of $1 \mu \mathrm{m}$ in diameter.

2. Wash with $1 \mathrm{ml}$ of $100 \%$ ethanol HPLC grade.

3. Vortex for $10 \mathrm{~min}$.

4. Let the particles settle down for $15 \mathrm{~min}$ at RT.

5. Give a short spin and take off the ethanol.

6. Wash with $1 \mathrm{ml} \mathrm{H}_{2} \mathrm{O}$.

7. Vortex for $1 \mathrm{~min}$ and let the particles settle down for $15 \mathrm{~min}$ at RT. Give a short spin at the end. Take off the $\mathrm{H}_{2} \mathrm{O}$ and repeat steps 6 and 7 three times more.

8. Take off the $\mathrm{H}_{2} \mathrm{O}$.

9. Resuspend the pellet in $1 \mathrm{ml}$ of $50 \%$ glycerol sterile.

10. Vortex for $10 \mathrm{~min}$.

11. Share in $15-20 \mu \mathrm{l}$ aliquots and store at $-20^{\circ} \mathrm{C}$.

DNA sample precipitation

1. Vortex the particles for 10sec.

2. Sonicate for $3 \mathrm{~min}$.

3. Add $10 \mu$ of DNA sample and vortex immediately.

4. Add $25 \mu$ of $\mathrm{CaCl}_{2} 2,5 \mathrm{M}$ and vortex immediately.

5. Add 10ul of spermidine and vortex 15-20 times. 


\section{Material and Methods}

6. Let on ice for $15 \mathrm{~min}$.

7. Take off supernatant and add $500 \mu \mathrm{l}$ of $100 \%$ ethanol HPLC grade.

8. Vortex for $10 \mathrm{sec}$ and let the particles settle down for $15 \mathrm{~min}$ on ice.

9. Give a short spin and take off the ethanol.

10. Add $200 \mu$ of $100 \%$ ethanol HPLC grade and vortex for 10 sec.

11. Let on ice for $15 \mathrm{~min}$.

12. Give a short spin and take off the ethanol.

13. Resuspend the particles in $10 \mu \mathrm{l}$ of $100 \%$ ethanol HPLC grade and keep on ice until use.

\section{Microprojectile bombardment}

1. Sterilize the metal carriers, metal web discs and sample membranes with $100 \%$ ethanol HPLC grade. Let them dry.

2. Assemble the metal carriers with the sample membranes.

3. Vortex the sample briefly, sonicate for $1 \mathrm{sec}$ and load this on two sample membranes (divide the sample). Let them dry.

4. Turn on the apparatus. Open the Helium bottle valve and set the pressure 200Psi more that the rupture membrane resistance. Turn on the vacuum compressor machine.

5. Assemble the apparatus carrier article by putting the following pieces in order: metal web discs, metal carriers and security tab.

6. Sterilise the rapture membranes (900Psi resistance) in isopropanol and let them dry.

7. Put the rupture membrane in the corresponding article of the apparatus and fix the last on the apparatus.

8. Put the carrier article at the upper possible position inside the apparatus.

9. Put the Petri plate with the callus material to be bombarded two positions lower than the carrier article (distance is a relative parameter and must be optimised in each situation).

10. Uncover the Petri plate and close the apparatus door. Turn on the vacuum. Once it reaches at $0,1 \mathrm{~atm}$ (find the corresponding position in each apparatus), block it.

11. Press the apparatus shoot button and maintain this pressed until the rupture membrane erupts.

12. Deactivate the pressure. Once it reaches the normal level (1atm) open the door and get the sample.

13. Incubate the samples on their Petri plates for 24 hours at $28^{\circ} \mathrm{C}$ in the dark.

14. Transfer the tissue slices to a microscope slide and mount with $\mathrm{H}_{2} \mathrm{O}$.

15. Visualize with a fluorescence light microscope or confocal microscopy (see section 2.4.4.2.6.1 and 2.4.4.2.6.2).

\subsection{Microinjection in oocytes and transport assays}

\subsubsection{Origin, management and injection of oocytes from Xenopus laevis}

The isolation, treatment and microinjection were performed following the protocols described by Bertran et al (1992a and 1992b).

\subsubsection{Transport assays of ${ }^{3} \mathrm{H}-\mathrm{IAA}$}

For each point of measurement 7 to 10 oocytes were injected. The oocytes were washed for 30 s with CholineCl medium. The transport were started incubating the 
oocytes in $100 \mu 1$ sample buffer at $25^{\circ} \mathrm{C}$ for the different transport intervals. After the transport interval the oocytes were washed 3 times with $4 \mathrm{ml}$ stop solution. Each oocyte is placed into a scintillation vial and dissolved with $200 \mu$ SDS $10 \%$. The measurement were carried out with LS6000SC (Beckmann)

CholineCl medium: $100 \mathrm{mM}$ CholineCl, $2 \mathrm{mM} \mathrm{KCl}, 1 \mathrm{mM} \mathrm{CaCl} ; 1 \mathrm{mM} \mathrm{MgCl} 2 ; 10 \mathrm{mM}$ Hepes adjusted to $\mathrm{pH} 5.5,7.5$ or 8.5 respectively.

Sample buffer: CholineCl medium $+2 \mu \mathrm{M}$ IAA $\left(30 \mu \mathrm{Ci} / \mathrm{ml} 3-\left[5(\mathrm{n})-{ }^{3} \mathrm{H}\right]\right.$-Indolylacetic acid, Amersham pharmacia biotech, UK).

Stop solution: $10 \mathrm{mM}$ CholineCl, $2 \mathrm{mM} \mathrm{KCl}, 1 \mathrm{mM} \mathrm{CaCl} 2 ; 1 \mathrm{mM} \mathrm{MgCl} ; 10 \mathrm{mM}$ Hepes adjusted to $\mathrm{pH} 5.5,7.5$ or 8.5 respectively

\subsubsection{Diffusion test for IAA in oocytes}

For the test of IAA diffusion the same experimental procedure were performed as described in the previous section. With the difference that the oocytes were incubated for 15 min with sample buffer containing different concentrations of radiolabeled IAA.

\subsection{Computer, software and servers}

Manipulation and analysis of nucleotide and amino acid sequences were performed using the following software and internet servers:

GCG: Genetics Computer Group Inc., Madison, Wisconsin, USA, 1991.

BLAST (EBI): http: / / www2.ebi.ac.uk/blast2/

BLAST (NCBI): http: / / www.ncbi.nlm.nih.gov/BLAST /

CLUSTALW: http: / / www2.ebi.ac.uk/ clustalw

DNA STAR software package: Madison, Wisconsin, USA.

ENDNOTE5, ISI Research Soft, Berkley, California, USA.

MIPS database: http: / / mips.gsf.de/

Monsanto rice database: http: / / www.rice-research.org/

MOTIF server: http:/ / motif.genome.ad.jp/

PHYLLIP: http: / / evolution.genetics.washington.edu/phyllip/software.html

PROSITE: http: / / www.expasy.ch/cgi-bin/\#pattern

PUBMED (NCBI): http: / / www.ncbi.nlm.nih.gov/PubMed/ 
TREEVIEW: http: / / taxonomy.zoology.gla.ac.uk/ rod/treeview.html

TMHMM-v2.0 server: http:/ / www.cbs.dtu.dk/ services/TMHMM-2.0/

TAIR database: http:/ / www.arabidopsis.org/home.html

SMART server: http: / / smart.embl-heidelberg.de/

SOSUI server:

http:/ / sosui.proteome.bio.tuat.ac.jp/cgi-bin/sosui.cgi?/ sosui_submit.html 


\section{Results}




\section{Results}

\subsection{Search for TM20 in several plants}

The comparison of nucleotide or amino acid sequences using databases is increasingly the first step to find out more about the function of a gene or a protein. When TM20 was cloned, no similar protein was found in the databases. However, with the rapid increase in number of sequences listed in the databases and the use of databases from the Monsanto Company (http: / / www.rice-research.org) has revealed amino acid sequences similar to TM20 in Oryza sativa, Arabidopsis thaliana and Hordeum vulgare.

\subsubsection{TM 20 in Oryza sativa}

The search of the entire amino acid sequence of TM20 (1389aa) against the six possible open reading frames (ORF) of the Monsanto database brought up several deduced protein sequences with identities up to $50 \%$ and similarities up to $63 \%$ between the TM20 query sequence and the blast result. The genomic clone with the accession number OSM117231 showed similarity with several parts of TM20 and the genomic sequence of OSM117231 lead to an ORF of 1129 amino acids with a calculated molecular weight of $126 \mathrm{KDa}$ for our protein that we call OsTM20 (Fig. 3.1).

The major part of the deduced protein (148aa -1129aa) is encoded by one exon in the genomic clone and it could be interpreted that OsTM20 is encoded only in one exon, as is the case for TM20. However, the lack of a hydrophobic region in the Nterminal of OsTM20 described for TM20 (Fig. 1.10), which plays an important role in the insertion process into the membrane suggested that this information might be encoded for OsTM20 in a second exon. The analysis of the genomic clone revealed a GC rich region upstream of the identified coding sequence of OsTM20. An exonintron prediction programme confirmed this GC rich region as a putative exon. Moreover, this region has similarity with the maize sequence and a hydrophobic region of 44 amino acids was identified in the deduced amino acid sequence coded by this second exon (Fig. 3.1). 


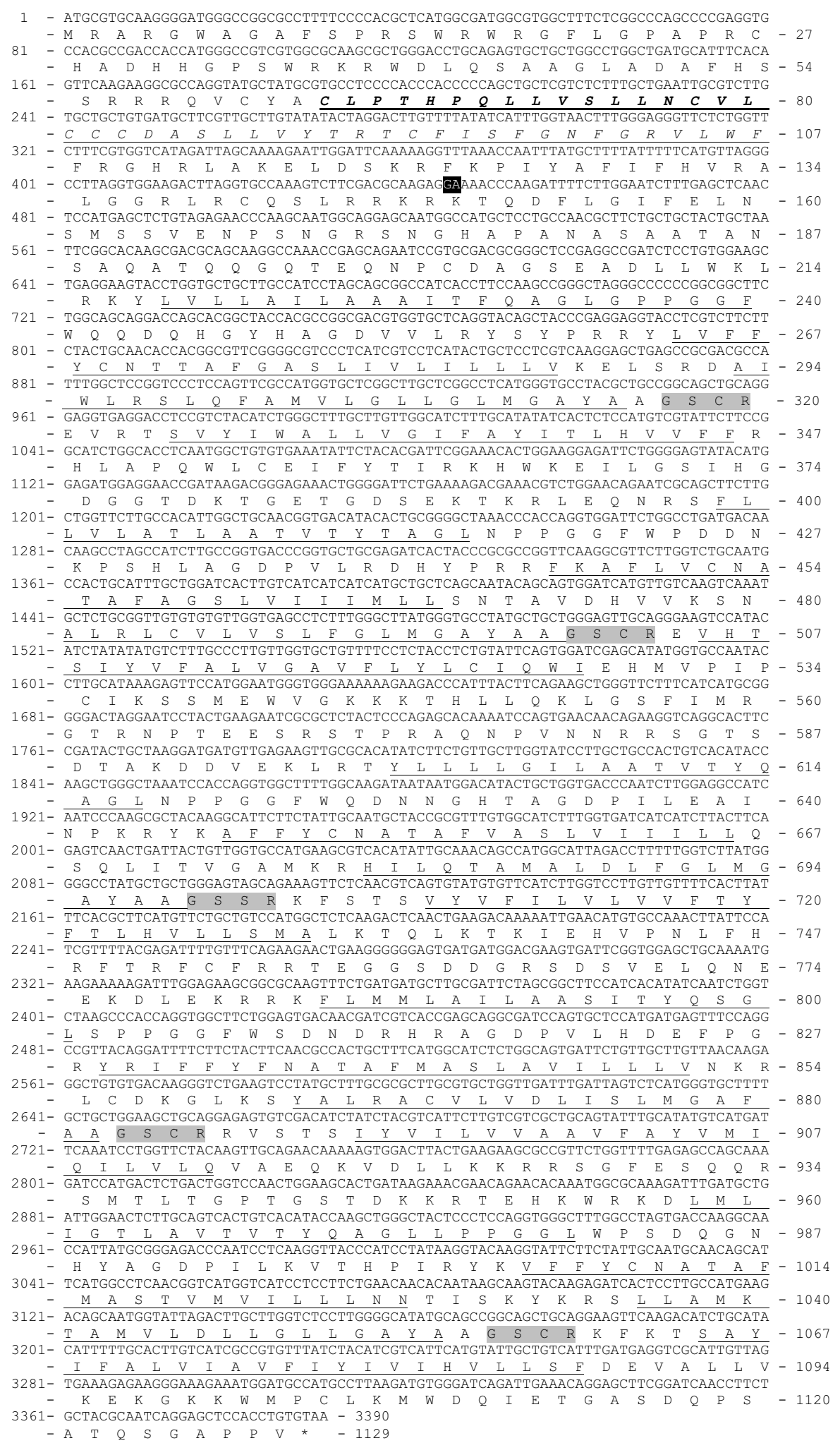

Figure 3.1: Sequence of the gene OsTM20 from Oryza sativa, which shares homology with TM20 and the deduced amino acid sequence from the genomic clone Acc.NoOSM117231. The predicted transmembrane domains are underlined and the putative phosphorylation sites for Kinase $\mathrm{C}$ are boxed. The numbers indicate the nucleotide position (left) and the amino acid position (right). A hydrophobic region in N-terminal is underlined and the corresponding amino acids are in italics. The position of the hypothetical intron (445bp-446bp) is boxed in black. 
Even if this result has to be confirmed experimentally, there is proposed that OsTM20 is encoded by two exons of $445 \mathrm{bp}$ and $2948 \mathrm{bp}$, which are separated by an intron of $1708 \mathrm{bp}$. In addition, the amino acid sequences of TM20 and OsTM20 from rice (Fig. 3.2) are $46 \%$ identical and the structure of both proteins is very similar.

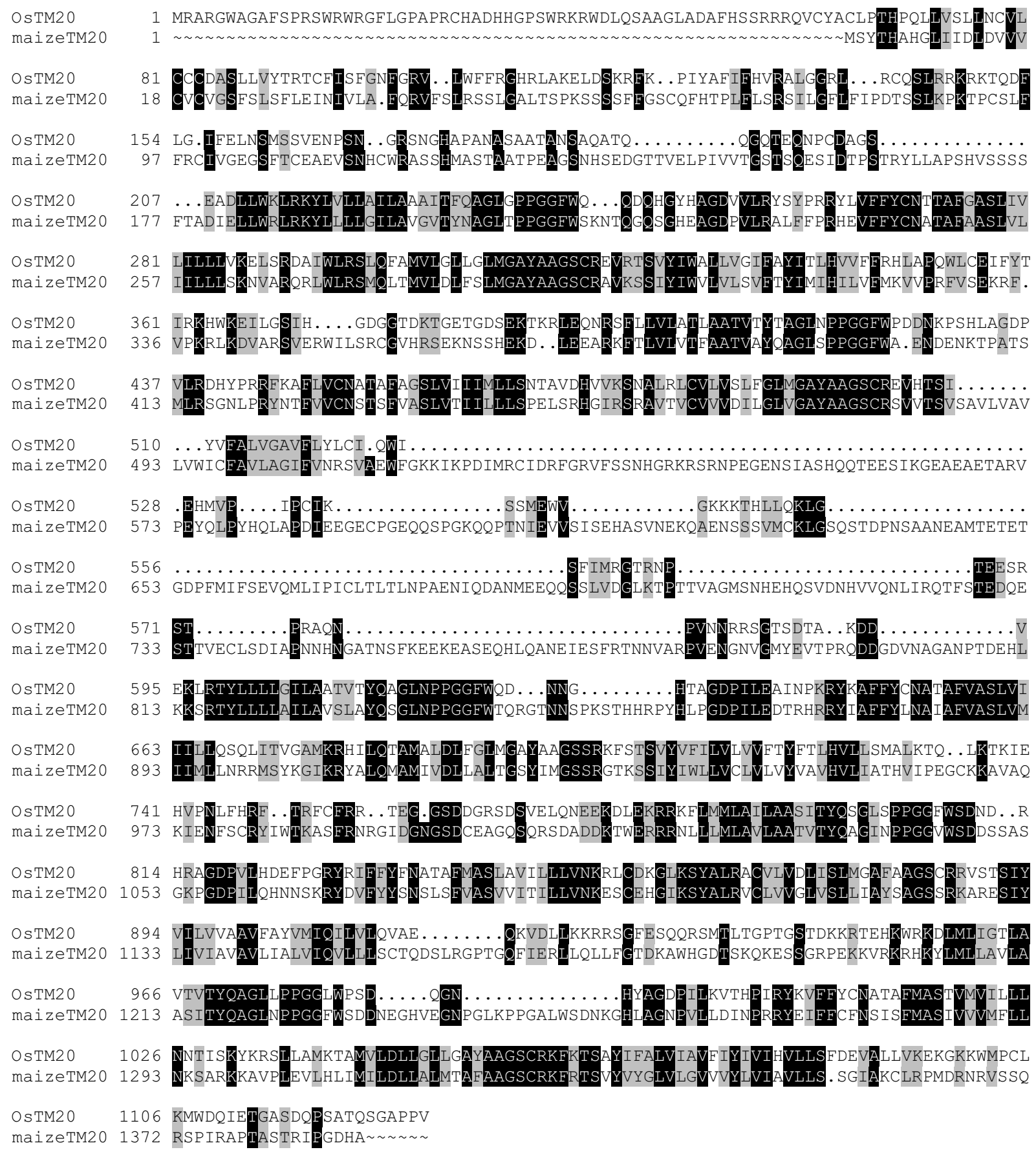

Figure 3.2: Alignment of the proteins from maize (TM20) and rice. Identical amino acids marked by black boxes and similar amino acids marked by grey boxes; identity (46\%) similarity (57\%). Alignment after Clustal W algorithm(Higgins and Sharp 1989) 
Applying the program TMHMM v2.0 for the prediction of transmembrane domains (http: / / www.cbs.dtu.dk/services/TMHMM-2.0/), it is possible to observe an equal number and equal distribution of transmembrane domains as observed in TM20 (Fig. 3.3, compare with Fig. 1.10).

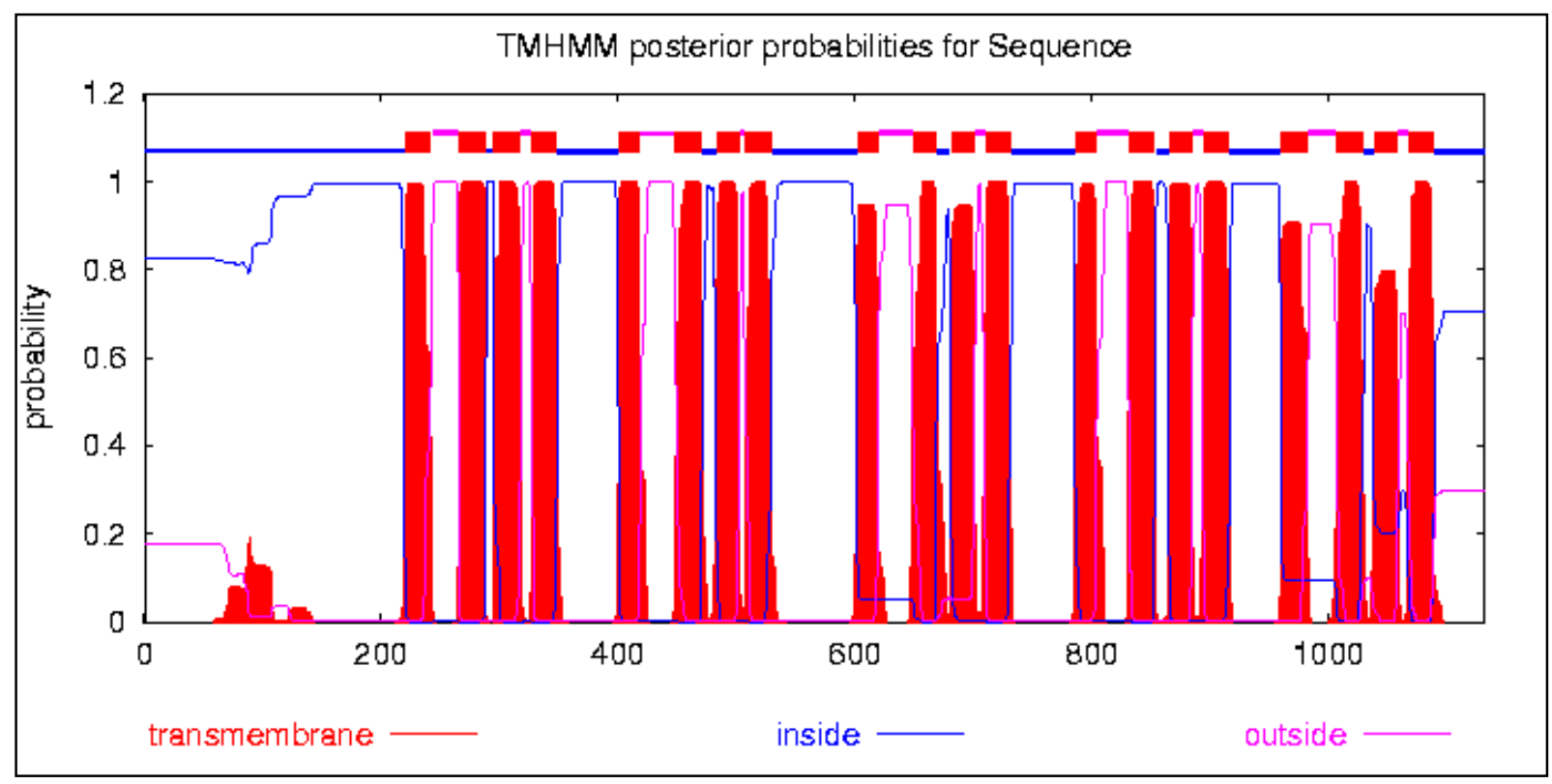

Figure 3.3: Schematic representation of the distribution of transmembrane spanning domains in the rice protein OsTM20 homologous to TM20 from maize. Graphic and prediction were calculated by the TMHMM v2.0 prediction server. Note the hydrophobic region at the N-terminal of OsTM20.

The rice protein possesses 20 transmembrane spanning segments grouped together in five groups of four transmembrane elements. The first transmembrane domain of every group is separated from the other three domains. The central loop described for TM20 (509aa-817aa, see Fig. 1.10) located between the second and third group of transmembrane domains is much shorter in the OsTM20 protein than in TM20 from maize. Beside the shorter central loop of the OsTM20 several gaps of 3 to 15 amino acids length are distributed throughout the last 450 amino acids (Fig. 3.2).

In addition five phosphorylation sites could be identified located, in the same position as described for the maize TM20 protein (Stiefel et al. 1999) (Fig. 3.1). The tetrapeptides GSC/SR are linked to the phosphorylation activity of protein Kinase C 
(Kishimoto et al. 1985; Woodgett et al. 1986) and were also described for proteins like the sodium dependent dopamine transporter from Bos taurus and Homo sapiens (Usdin et al. 1991; Giros et al. 1992).

The results of these analyses suggest that a protein structurally similar to TM20 exists also in Oryza sativa, sharing the characteristics of a transporter protein and may fulfil the same function as TM20.

\subsubsection{TM20 in barley (Hordeum vulgare)}

Evidence for the existence of a TM20-like protein in barley (Hordeum vulgare) is given by the homology shared with a $245 \mathrm{bp}$ fragment of genomic DNA of barley. This fragment was found by running a tblastn, which compares a query amino acid sequence (in this case TM20) against the six possible open reading frames of a nucleotide databank. The DNA sequence with the accession number AJ234475 was found by Michalek et al (1999) working on gene identification in RFLP markers in barley (Michalek et al. 1999). The protein sequence of 81 amino acids deduced from this DNA fragment has an identity index of about $67 \%$ and a similarity index of $74 \%$ with TM20 maize protein (Fig. 3.4). Due to the high identity index and the evolutionary proximity of barley and maize, it can be suggested that TM20 will also be found in barley.

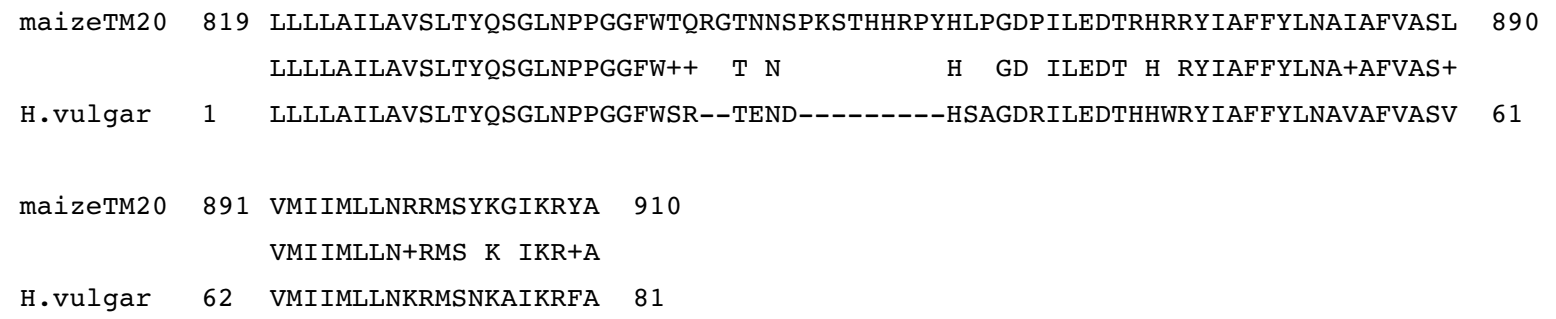

Figure 3.4: Alignment between the amino acid sequence of TM20 of maize and a deduced protein sequence from barley found with a tblastn search. The barley protein sequence is deduced from a fragment of genomic DNA, introduced in the databanks by Michalek et al (1999). Acc. No AJ234475. 


\subsubsection{TM20 in Arabidopsis thaliana}

Performing protein-protein homology searches (blastp) with the entire amino acid sequence of TM20, several proteins with a low index of homology were found. The protein with the highest index of homology with TM20 is encoded by a gene, which is placed on chromosome 4 of A.thaliana. The protein At4g11000 was classified as putative protein. Figure 3.5 shows the region of highest homology between TM20 and At4g11000. Since this region of homology is repeated with modifications 5 times in the amino acid sequence of TM20 (see introduction), At4g11000 was aligned with the blast program 5 times against these repeats (not shown).

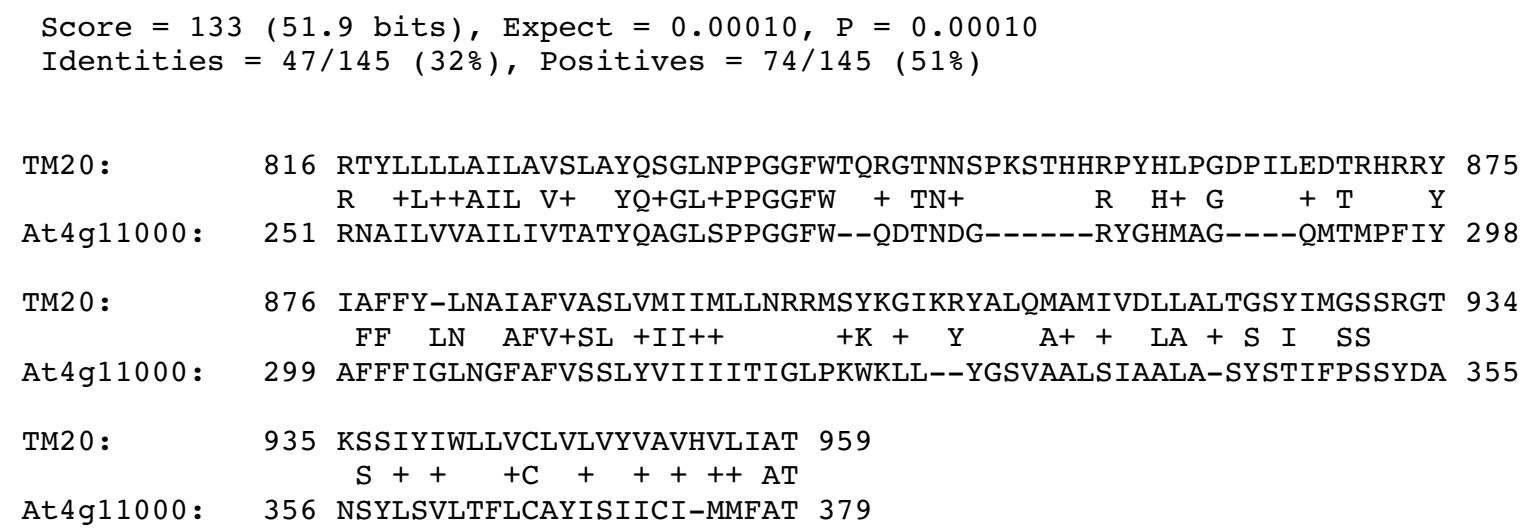

Figure 3.5: Alignment of a part of the amino acid sequence of TM20 against the putative protein At4g11000 of Arabidopsis showing the region of highest homology. Result of a protein-protein blast in the NCBI and Ebi database.

The protein At4g11000 was also indicated as "related sequence" to TM20 using motif and pattern search programs like Smart (Schultz et al. 1998; Schultz et al. 2000) (http:/ / smart.embl-heidelberg.de) and Motif (http://www.motif.genome.ad.jp), programs, which scan amino acid sequences for known motifs and compare the query sequence to proteins which possess these motifs too.

The introduction of the amino acid sequence of At4g11000 into the program TMHMM v2.0 to predict putative transmembrane domains brought up four transmembrane spanning domains, located at the C-terminal of At4g11000. These four membrane domains were grouped together in the same manner as one of the groups of four transmembrane segments described for TM20 (Fig. 3.6). 


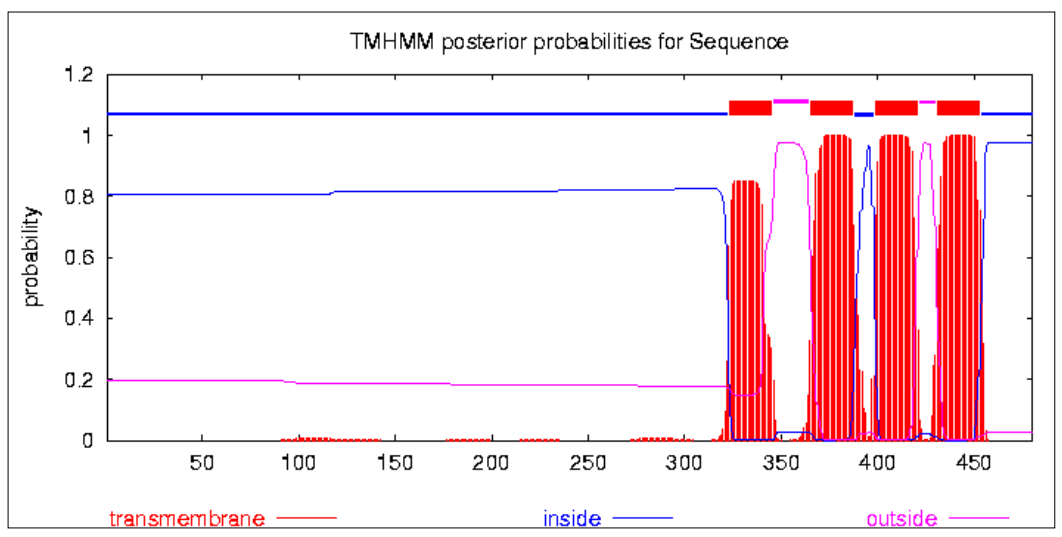

Figure 3.6: Schematically representation of the probability of transmembrane spanning domains in the Arabidopsis protein At4g11000 similar to TM20 from maize. Graphic and prediction are the output of the TMHMM v2.0 prediction server.

Using RT-PCR with primers designed to amplify the gene coding for At4g11000, the corresponding DNA could be amplified and cloned. For the retrotranscription, RNA extracted from young plants of A.thaliana ssp Columbia was used. The PCR-product obtained had a size of about $1430 \mathrm{bp}$, which corresponds to the size of the predicted gene encoding At4g11000. The product of the RT-PCR was entirely sequenced and compared to the nucleotide sequence of At4g11000 from the database. As shown in Figure 3.7 the gene of At4g11000 is a compound of three exons and two introns. Within the first intron a 367bp duplication of the $5^{\prime}$ end of the coding sequence including the ATG was found (Fig. 3.7). This duplication is spliced out during the process of transcription. However, an alternative splicing can not be excluded, although the RT-PCR detected only one gene product. The protein encoded by this gene will be termed AtTM4. 


\section{Results}

81001 gcaagaccag ctcaaattac ctctgtgtac taactgtgga aaggttggtc atactgtgca 81061 gaagtgttac aaaatcattg gctatcctcc tggctataag gcagctacat catacaggca
81121 acctcaaata caaactcagc ctagaatgca gatgccacaa caatctcaac caaggatgca 81181 acaacctgta gcagcacaaa tgcagacaaa catgccaaat acaggaccaa tgcagatgat 81241 cccttatgca aatcaaatgc agatggttcc atactcgaat tcaatgcaga tggcgaatgc 81301 tgcttatgct gaataaggtt cttttgttcC aatgATGccg catgttacca gtggaggtaa 81361 taatctaaac ttgcaagatc tttctcaaga tcagatacaa catcttattt cacaattcaa 81421 tgctcaagtt cgggttcaag agcctgcagc tacttctatt tatacctcta gtcctactgc
81481 tactattact gaacatggct tgatggcaca aacatctact tctggtacaa ttccttttcc 81541 ttctactagc ttgaatatg aaataata tctcaccttt cagatcata ctctttcttc 81601 cttacaaaat gtttatcaa gtgatgcttg gattattgat agtggtgctt ctagtcatgt 81661 ctgttcggat ttaacaatgt ttagggaatt aatacatgtg tetggtgtaa ctgttacatt 81721 accaaatggt actagggttg caattacaca cacaggaacc atttgcatca cgtctactct 8181 cattctgcat aatgttttac ttgttcctga tttcaaatt aacttgatta gtgtctgttg 81901 tctgttaaa accttgtcte actcggctca tttctttgct gattgttgtt atatccagga 81961 gacacaacgt acatccttct caccatcact acctgcagct agttcattca ctggaaccgt 82021 tcaagatgat tgtcttctgt ggcaccagcg tctaggcatc cgtcattacc tgcattacag 82081 aaacttgtta gttcaattcc ttctttaaag tctgtttcat ctactgcttc gcattgtaga 82141 attagtcctt tagctaaga 8201 agtccatttg atttgattca tttagacatt tggggtcctt ttagtattga gtctgttgat 82261 ggttttagat acttcttaac tttggttgat gattgtacaa gaaccacttg ggtttatatg 82321 atgaaaaata aaagtgaagt ttccaatata tttcctgtct ttgttaagct cattttcact
82381 caatataatg ccaaattaa agcaattagg tctgataatg taaaagaact agcttcacc
82441 aaatttgtta aagaacaagg aatgatacat caattttctt gtgcttacac tcctcaacag 82501 aattctgttg ttgagcgtaa gcatcaacac ttgttaaaca tagctaggtc tttattattt 82501 aattctgttg ttgagcgtaa gcatcaacac ttgttaaaca tagctaggtc tttattattt
82561 cagtcgaatg tacctttgca atattggagt gattgtgttc ttactgcagc ttatttgatt 82561 cagtcgaatg tacctttgca atattggagt gattgtgttc ttactgcagc ttatttgatt
82621 aatcgtttgc cttctccatt gttagacaac aaaactccat ttgaacttct tttaagaaa
82681 attccagatt atactcttt gaaaagttgt ctttgctatg catcaactaa tgttcatgat 82741 aggaataagt ttagtcctag agctagacca tgtgtgtttt taggatatcc ttcaggatac 82801 aaaggttata aggttttaga tttagagtct cattccattt ccattactcg taatgtggtt
82861 tttcatgaaa caaagtttcc atttaaaaca agtaaatttt taaaagaatc tgttgacatg 82861 tttcatgaaa caaagtttcc atttaaaaca agtaaatttt taaaagaatc tgttgacatg
82921 tttcctaatt ccattttacc attgcctgca ccattgcatt ttgtagaaag catgcctctt 82921 tttcctaatt ccattttacc attgcctgca ccattgcatt ttgtagaaag catgcctctt
82981 gatgatgatt tacgtgctga tgataacaat gettctacat ctaattctgc atcatctgca 83041 tcatctattc cacctttacc tagtactgtg aatactcaga acacagatgc attagatata 83101 gatactaatt ctgtacctat tgctagaccg aaaagaaatg ctaaagcacc tgcttatttg 83161 tctgagtatc actgtaattc ggttcetttt ctttcttcac tttcacctac tacttctact
83221 tecattgaaa caccatcatc atctattcca cctaaaaga tcactactcc atatcctatg 83281 tccacagcca tttcctatga taaacttact ccactttttc attcttatat atgtgcttat 83341 aatgttgaaa ctgagccaaa ggcttttact caagccatga aatctgaaaa gtggactaga 83461 actgagggta aaaatgttgt aggttgcaag tgggttttca caataaagta taaccccgat 83521 ggaagtattg aacgatataa agcacggttg gttgctcaag gctttactca gcaggagga 83581 attgattata tggagacttt ttcacctgtg gcaaagtttg gcagtgtgaa attgttactt 83641 ggacttgctg ctgcaactgg ttggagctta acgcagatgg atgtttctaa tgccttctta 83701 catggagagc ttgatgagga aatctacatg agtctgccac aaggttatac tccaccaact 83821 caagcatcta gacagtggta taagagatta tcttcagtct tcttgggagc aaactttatt 83881 cagtcacctg cagataacac tatgtttgtg aaagtcagtt gtacttcaat cattgtggtg 83941 ttagtctatg ttgatgactt gatgattgca agtaatgata gctcagcagt ggagaatttg 84001 aaagaattat tgagatctga getcaaaata aaggatttag gaccagctag atttttcta 84061 ggattggaga ttgctagatc ttctgaggga atttctgtct gtcaacgcaa atatgctcaa
84121 aatttgcttg aagatgtagg gctttctggt tgtaaaccaa gctcaatccc catggatcct 84181 aacctgcacc tgactaaaga gatgggaacc ttactgccaa atgcgacttc ctatagagag 84241 cttgttggac gattgttata cttgtgcatt actcgtcctg atataacttt tgcagtacat 84301 actttgagtc agtttctctc tgcaccaact gacattcata tgcaagctgc tcacaaggtt 84361 ctgcgatatc tcaaaggtaa tcctggtcag ggtttgatgt attctgcttc ttctgagttg 84421 tgtttaaatg gtttctcaga tgctgattgg ggtacttgca aagattcaag gagatctgtt
84481 actggttttt gcatttatct tggtacatct ttgattacat ggaagagtaa gaagcaatct 84541 gtggttagtc ggagtagtac agaatctgaa tatagaagct tagcacaagc cacttgtgag 84601 attatctggt tgcaacagct gctcaaagac ttgcatgtga ctatgacttg tecggctaaa 84661 ctcttttgcg acaacaaatc tgcactacac ttggcgacga atccagtctt tcatgagcgt 84721 accaagcata ttgaaatcga ctgtcacact gtaagagacc aaatcaaggc tggaaaactg 84781 aagactctac atgttccaac aggaaaccag cttgcagaca ttctaaccaa gccacttcac 84901 caacagcaag gaagataaga tttgaggggg gtgtattggt gattggttta atctggttaa 84961 ctccggtt 85021 tagtttattt tgttgatgaa cgttgtaaca aacacttaat tetgttaata acgattcagt 85081 tcaatctccg atcttctctc ttcttttccg tttcaccgga acttcgccgg tgttaatagt 85141 ggaggcagca gaagcttggA TGcctagtgt agctatgtcg attgttgtgg atattagtag agtatcagat tcggtagctc aaaatgcaaa tttgagtcgg agttgggtac gagcactcaa 85321 tacgagttga tagcggaaga tccaaacatt ttagaccact ttgatcaagt atctttttgt 85381 gagacgccac tacacattgc agctgaaaa gggcaaactc atttcgccat ggaactaatg 85441 actcttaagc cgtctettgc tttgaagcta aacgtgttag gtttcagcce actgcattta 85501 gccttacaaa acaaccacat tcgaacggtg agagggttag tggcaatcaa cagcagctta 85621 gttagcata agagat 85681 aagtgtgaga cagaagattg caatcttcag gecagctcga gctccggtga aaccttgatg 85741 atttcctttt gctgcttcct cttcacgcac cagaagaggc agccaccaac caccagtagg
5801 gcaaagatgc ccaacaagat caggtagaat tgtcctggta gcaataatag ataggatcca 85861 tcatattgg tccgctctcc atctaaaaat ttatctagat attgttctta atctttgttc 85921 taagaagtgt ttgattcaat aaacatatct tggttgctca atgatgtctt tccgaggagt
85981 caagaagtag agcgcaaatt tcgattggaa attgtttcgc tgagtttgtt ttatgaagta 86041 atggtttgtt ttgttttgaa ctttgaaggg taactaacag tctaataaat cttagtgctc
86101 taacactaaa ttagttatac tttaggacac aagtaaagta taactaactg tctaataagt
86161 cttagtgctc taacactaaa tttccacaat ccatctcttg atatcttcac gtcattcacc 86161 cttagtgctc taacactaaa tttccacaat ccatctcttg atatcttcac gtcattcacc
86221 ggccagctcg agctceggta aaatcttgat gatttgtttc tgctgctagc ttcctcttca 86221 ggccagctcg agctceggta aaatcttgat gatttgtttc tgctgctagc ttcctcttca
86281 cgcaccagaa gagcgtgaag aggaagcaac caaccacaag TGAggcaagg atgccaacaa 86281 cgcaccagaa gagcgtgaag aggaagcaac caaccacaag TGAggcaagg atgccaacaa 86401 aaacatctac aaaagttaa tattaggcta atgggattac ccctttgata tttaatggat 86461 gtctcttggc gcagtgtttt tgaagacact cggtcattcc ggcgtttcgt ttattagatg
86521 atattcagt tatttttgga tatttggat atttctagt atttactatt tttatatatt 86581 tatcaagtat tttggattat ttggtctata aataactaat atttttagat atataattat 86641 atttcgaata ttttcatata tccaaaatat tttggttctt tcggttatag aatttggtat 86701 tcattcggtt attttcaaat ttttggttcg attcggttaa tgacctaaat tgctactaat 86821 catgggttca catgcaagag gcatagcgat caaaaattaa tcaaaaatta catatcttaa 86881 ttttacgtga gttattggtc aattagatat tgacatgtat gaaattattt cttttaaaaa 86941 aatttttagc atggatatta attaattaca attaatattg aaattcaaaa tagcaatata 87001 aatgatttaa cattgattta attggataa aagttgtatt tatcgttata atataatatt 87121 gaatcacta taacgctagt gaaagatatt tttaattaat gtcattatcc atctagttgt 87181 catattattg ctagacagaa gtgtatggac ttagttgacc tgaataaga atagtcaaag
87241 tcttttatgt ggtggacctg aaattagact aatcaaagtc tttgtgttta ctttgtagtt

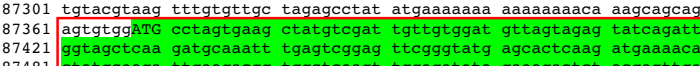
87541
87601 7661 gtcacttgct ttgaagctaa acgtgtcagg tttcagccca ctgcatttag ccttacaaaa 87721 caaccacatt caaacggtga gagggttagt ggcaatcaac agcagcttag ttagcatata gattaatca gactgagta agagaatcat tttggtccat 87961 gaaaatggat aaatatgttc ccatgacctt agtataggca ttttattctt atcagtgaag
88021 gattctttat cctcgaattg ttggttgagg tgtagtcaat ttttgcaggt gatgaaattg

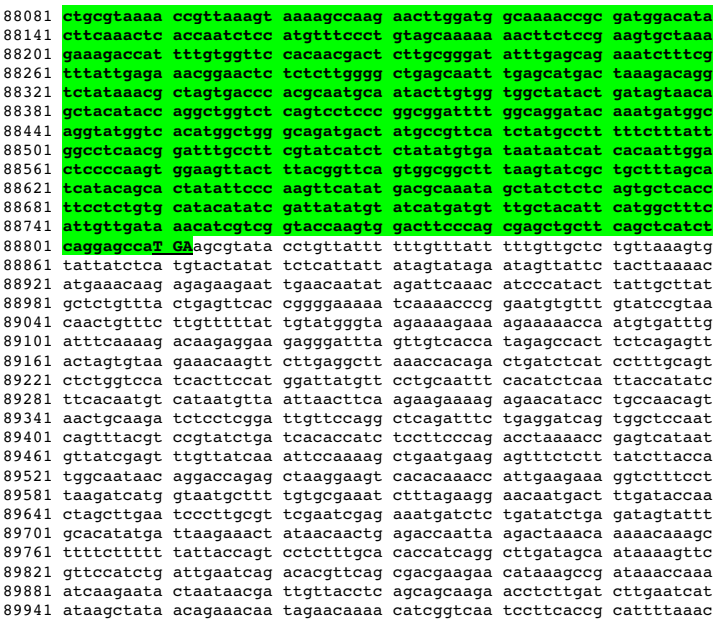

Figure 3.7: Part of the genomic sequence of Bac F25I24 containing the two predicted genes At4g10990 and At4g11000. The exons of the genes predicted by the genome project are marked in yellow (At4g10990) and green (At4g11000). The exons of the cDNA are marked by the dark green bars at the side of the genomic sequence and represent the sequence amplified by RT-PCR, written in bold. A duplication of the genomic sequence is marked by red boxes. 
With the purpose of obtaining more information about the transcription sites of this gene, RT-PCR analysis was carried out on different tissues from adult plants of A.thaliana ssp. Columbia. RNA was extracted from leaves (L), ground leaves (GL), stem (St), basal part of the stem (St2), middle part of the stem (St3) and siliqua (S1). In addition, RNA from plantlets $(\mathrm{Pl})$ and from root of the plantlets $(\mathrm{R})$ was used for retro-transcription into CDNA and PCR-amplification. Except for the siliqua a band of approximately $1430 \mathrm{bp}$ was found in all analyzed tissues (Fig. 3.6). This result shows that the transcript of At4g11000 is present in nearly the whole plant, except in the siliquas.

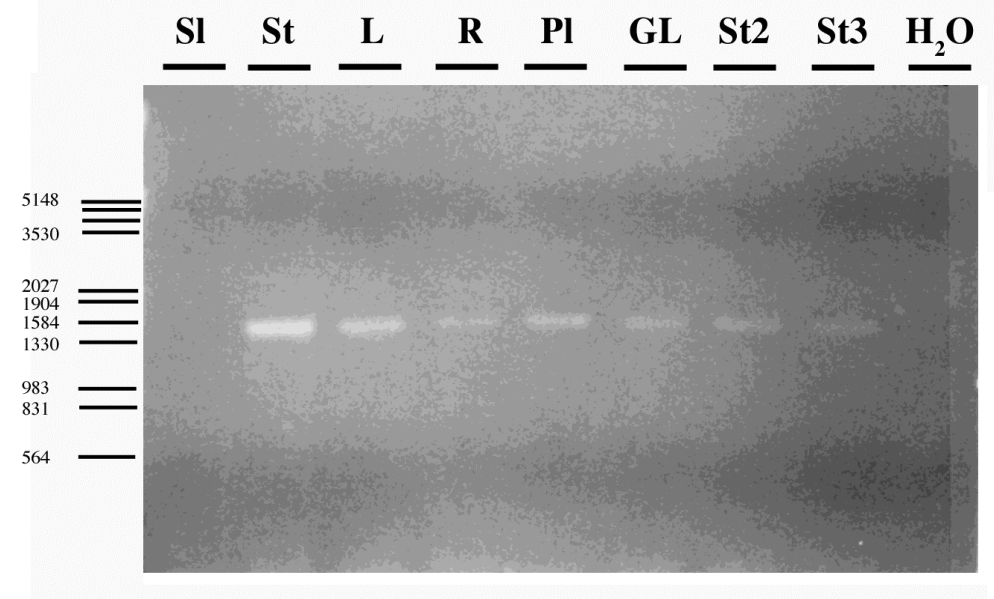

Figure 3.6: $R T-P C R$ on RNA of different tissues of A.thaliana amplifying a PCRproduct of about $1430 \mathrm{bp}$. Primers employed in this RTPCR were used before to clone the cDNA of At4g11000. (Sl) siliqua; (St) shoot; (L) stem leaf; (R) plantlet root; (Pl) plantlet; (GL) ground leaf; (St2) basal part of the shoot; (St3) middle part $\mathrm{p}$ of the shoot; $\left(\mathrm{H}_{2} \mathrm{O}\right)$ control PCR without cDNA template.

Since the homology between TM20 from maize and AtTM4 is relatively low, a new search in the databases of A.thaliana was started, this time using the amino acid sequence of AtTM4 as query sequence. A variety of genes coding for putative proteins were found in this search located on different chromosomes (Table 3.1).

The genes Arab2 and Arab5 on chromosome 1 are placed in tandem, whereas AtTM4 and Arab4 on chromosome 4 are separated by approximately $122 \mathrm{~kb}$. On chromosome 5 are localized three genes, Arab1 at approximately 5,1 Mb and Arab3 and Arab6 at $22 \mathrm{Mb}$. The two latter genes are placed also in tandem (Fig. 3.8). A comparison with the map of the duplicated chromosome regions of the Arabidopsis genome showed that the predicted genes are not situated in regions of known 
chromosome duplications. For this reason and because of the homology between them, these genes may be members of one gene family.

\begin{tabular}{|c|c|c|c|c|c|c|c|}
\hline Name & Acc. No. MIPS & Bac & Chr. & Length & AA & ProteinMW & ESTs \\
\hline Arab1 & At5g15500 & $\mathrm{T} 20 \mathrm{~K} 4$ & V & $1371 \mathrm{bp}$ & 457 aа & $51,5 \mathrm{KDa}$ & BE038658 \\
\hline Arab2 & At1g14480 & F14L17 & I & $1323 \mathrm{bp}$ & 441 aа & $49,9 \mathrm{KDa}$ & $\begin{array}{l}\text { AV537342 } \\
\text { AV549133 }\end{array}$ \\
\hline Arab3 & At5g54620 & F24B18 & V & $1287 \mathrm{bp}$ & 429 aа & $48,4 \mathrm{KDa}$ & None \\
\hline Arab4 & At4g10720 & $\mathrm{T} 12 \mathrm{H} 20$ & IV & 1332 bp & 444 aа & $49,5 \mathrm{KDa}$ & None \\
\hline Arab5 & At1g145003 & F14L17 & I & 1308 bp & 436 аa & $48,8 \mathrm{KDa}$ & $\begin{array}{l}\text { AA585892 } \\
\text { AV564001 } \\
\text { AV565179 }\end{array}$ \\
\hline Arab6 & At5g54610 & F24B18 & V & $1275 \mathrm{bp}$ & 425 aа & $48,3 \mathrm{KDa}$ & None \\
\hline AtTM4 & "At4g11000" & $\mathrm{F} 25 \mathrm{I} 24$ & IV & 1432 bp & 480 aа & $52,7 \mathrm{KDa}$ & $\begin{array}{l}\text { AV531008 } \\
\text { AV538348 } \\
\text { AV545504 } \\
\text { AV546658 }\end{array}$ \\
\hline
\end{tabular}

Table 3.1: List of the genes found in Arabidopsis encoding proteins, which share homology with AtTM4. All genes code for putative proteins with four transmembrane domains on the C-terminal part grouped together as in TM20, OsTM20 and ArabTM4. The different genes are located on different chromosomes and vary slightly in length. The Acc.no. for AtTM4 corresponds to the predicted protein, but the length and MW of the protein corresponds to the sequence amplified by RT-PCR.

Basically there are two positions of introns that are conserved in Arab3 and Arab6. Another gene that also possesses two introns is AtTM4. The second intron corresponds in its position to the second intron of Arab3 and Arab6, while the first intron AtTM4 varies compared to the first intron of Arab3 and Arab6 (Fig. 3.8 and Fig. 3.10). In contrast, the other genes, Arab1, Arab2, Arab4 and Arab5 possess only one intron, whose localization corresponds either to the position of the first or the second intron of Arab3 and Arab6 (Fig. 3.8). This result indicates that in Arab1, Arab2, Arab4 and Arab5 one intron has been deleted during evolution. In this context, the genes placed in tandem (Arab2:Arab5 and Arab3:Arab6) maintained the number of introns respective to the tandem partner. The conservation of the described exon-intron structure is additional evidence that these genes probably belong to one gene family. 


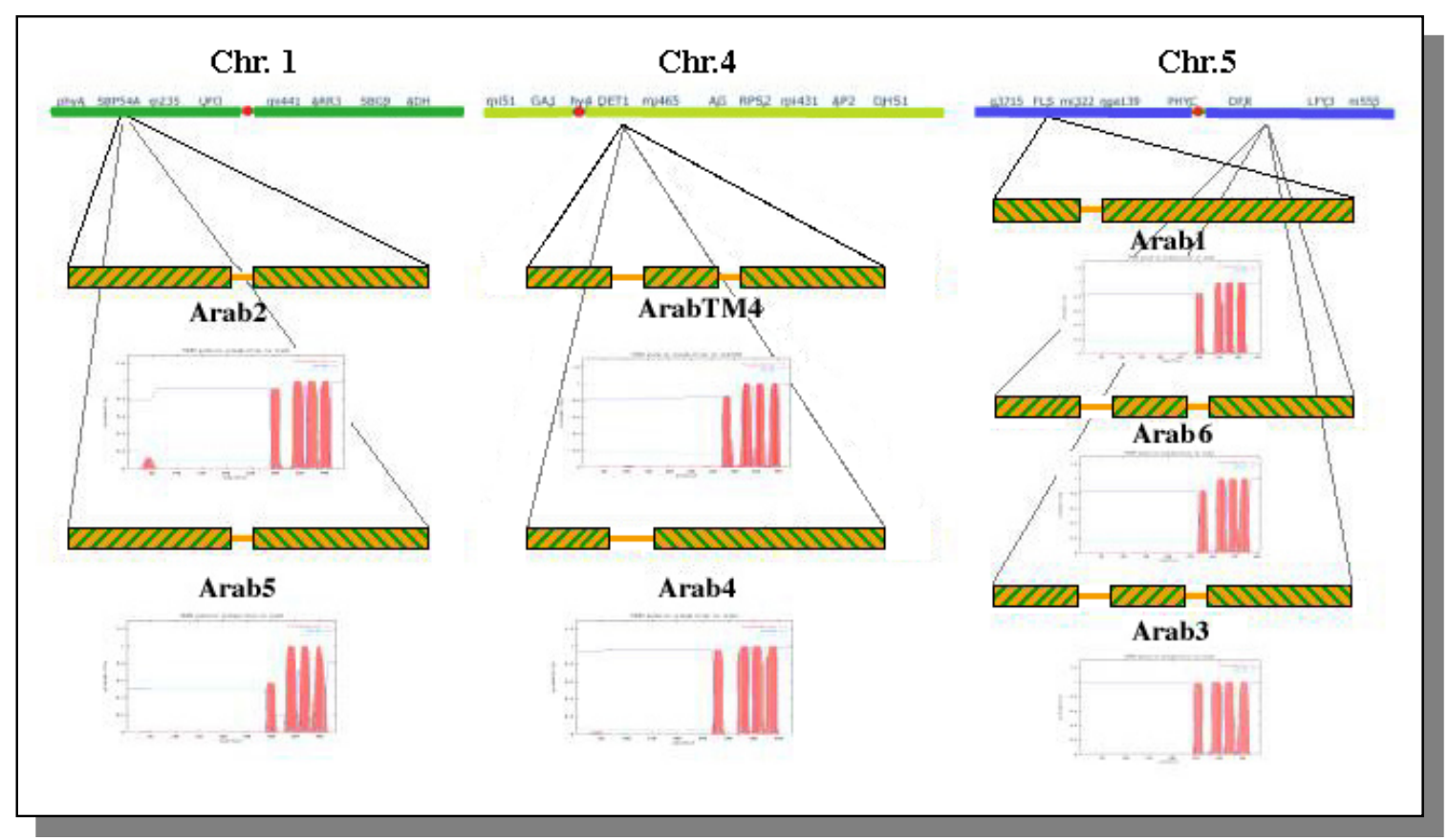

Figure 3.8: Graphical overview of the genes Arab1-Arab6 and AtTM4 found in the databases of the Arabidopsis genome, and their chromosomal localizations. Every exon is presented by striped bars and the introns are documented by thin yellow lines. The respective predictions of the transmembrane domains calculated by the TMHMM v2.0 server for the deduced proteins are represented below the gene names.

As presented in Figure 3.8, for all these proteins transmembrane segments were predicted and in all cases the same group of four transmembrane spanning domains could be found. The other characteristic feature shared by all the described proteins is the presence of various ankyrin repeats in the N-terminal part of the proteins (Fig. 3.9). Ankyrin repeats are modules of about 33

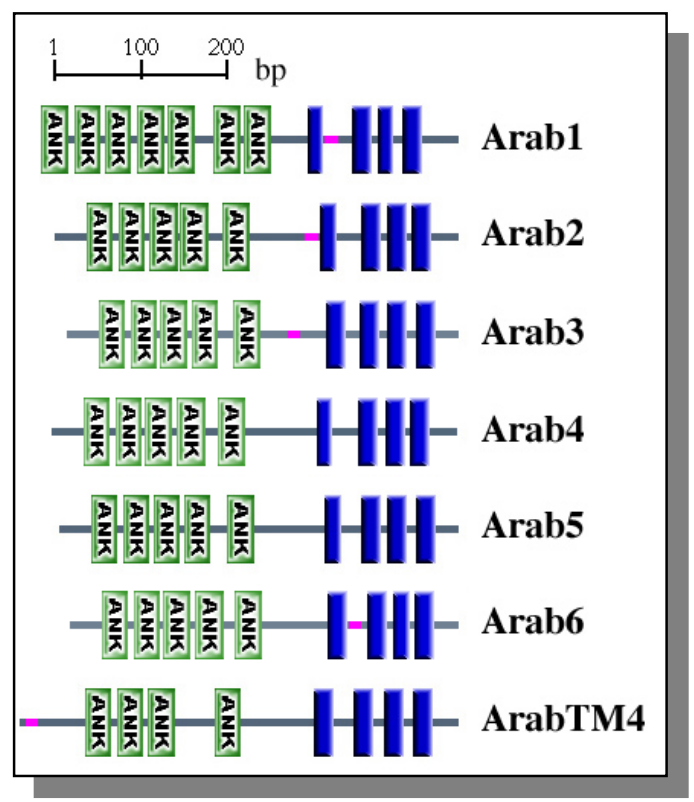

Figure 3.9: Motif predictions for the proteins Arab1-Arab6 and AtTM4. Prediction was made by the Smart server tool (http://www.emblheidelberg.de). Blue bars represent the putative transmembrane domains. Green bars indicate the position of the different predicted ankyrin repeats. The number of Ankyrin repeats is variable whereas the number and position of transmembrane spanning domains is the same in all proteins. 


\section{Results}

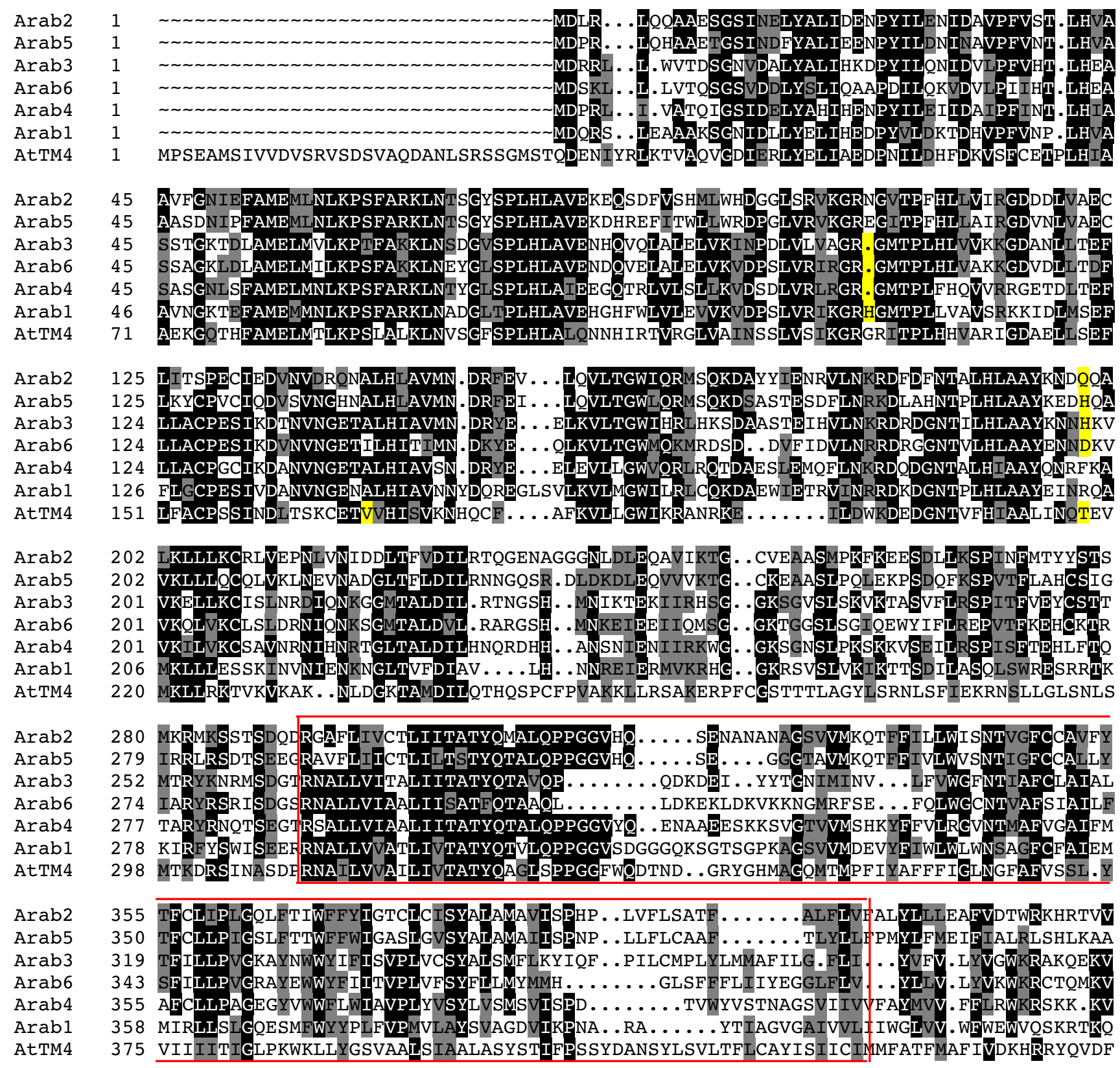

Arab2 426 PKSRFSWFWKVLQYYYIP 444

Arab5 421 VNRMFVTFVKORGTLRIP 438

Arab3 392 PEPKSELTFEGFTSMTP 408

Arab6 411 RKPKSDLISDNFKNMVP 428

Arab4 425 PGTSSELILEGLTTLDLAKGSP 446

Arab1 429 RGPKSGLVWEGFTTLDQARGVAPNRYVIRT 458

AtTM4 455 PASCFSSSQEEPVIQA 470

Figure 3.10: Alignment of the seven proteins found encoded in the Arabidopsis thaliana genome. Identical amino acids are marked in black and similar amino acids are boxed in grey. There is a higher homology found in the N-terminal than in the C-terminal. The region of homology with TM20 is boxed in red. The positions of the introns are marked in yellow. 
amino acids and occur in at least four consecutive copies. Many ankyrin regions are known to function as protein-protein interaction domains. The number of ankyrin repeats ranges from 4 to 7 in the predicted proteins Arab1-6 and AtTM4. The predictions of the ankyrin repeats were found by applying the Smart server prediction program (http:/ / smart.embl-heidelberg.de) to identify known protein motifs in amino acid sequences.

The alignment of the amino acid sequences showed a relatively high homology between the different proteins (Fig. 3.10) especially in the N-terminal part of the proteins. In contrast the C-terminal is characterized by more variability. However, the structure of the proteins and the homology of the amino acid sequence indicate that the proteins are related. Concerning the homology to TM20, these proteins are the most similar to TM20 in the genome of Arabidopsis. The difference may be an indication of the variation found in genes from monocots and dicotyledons.

\subsection{Characterisation of maize TM20}

Three approaches have been followed in this work to characterize the maize protein TM20. In the first approach, antibodies against TM20 were generated with the aim of characterizing TM20 with immunological methods including western blot and immunolocalization in embryo tissues. For the second approach a fusion protein of the N-terminus of TM20 with GFP was used to transfect onion epidermis cells. The third approach focused on the expression of TM20 in oocytes of Xenopus laevis to perform transport studies with radiolabeled IAA.

\subsubsection{Generating antibodies}

In this work five antibodies were generated against different zones of the protein TM20 to identify the expression pattern of TM20 in embryo tissue and to locate the protein at the subcellular level. To produce antibodies against the entire protein were not considered due to its size, which would complicate the overexpression and purification process in E.coli. Moreover the protein has 20 
transmembrane spanning domains, which are characterized by low antigenicity. Figure 3.11 represents schematically the recognition sites of the antibodies on a model of TM20. For the production of the different antigens two strategies were followed. In the first case the loop region was expressed in E.coli and in the second case synthetic peptides of 15 amino acids length were acquired commercially.

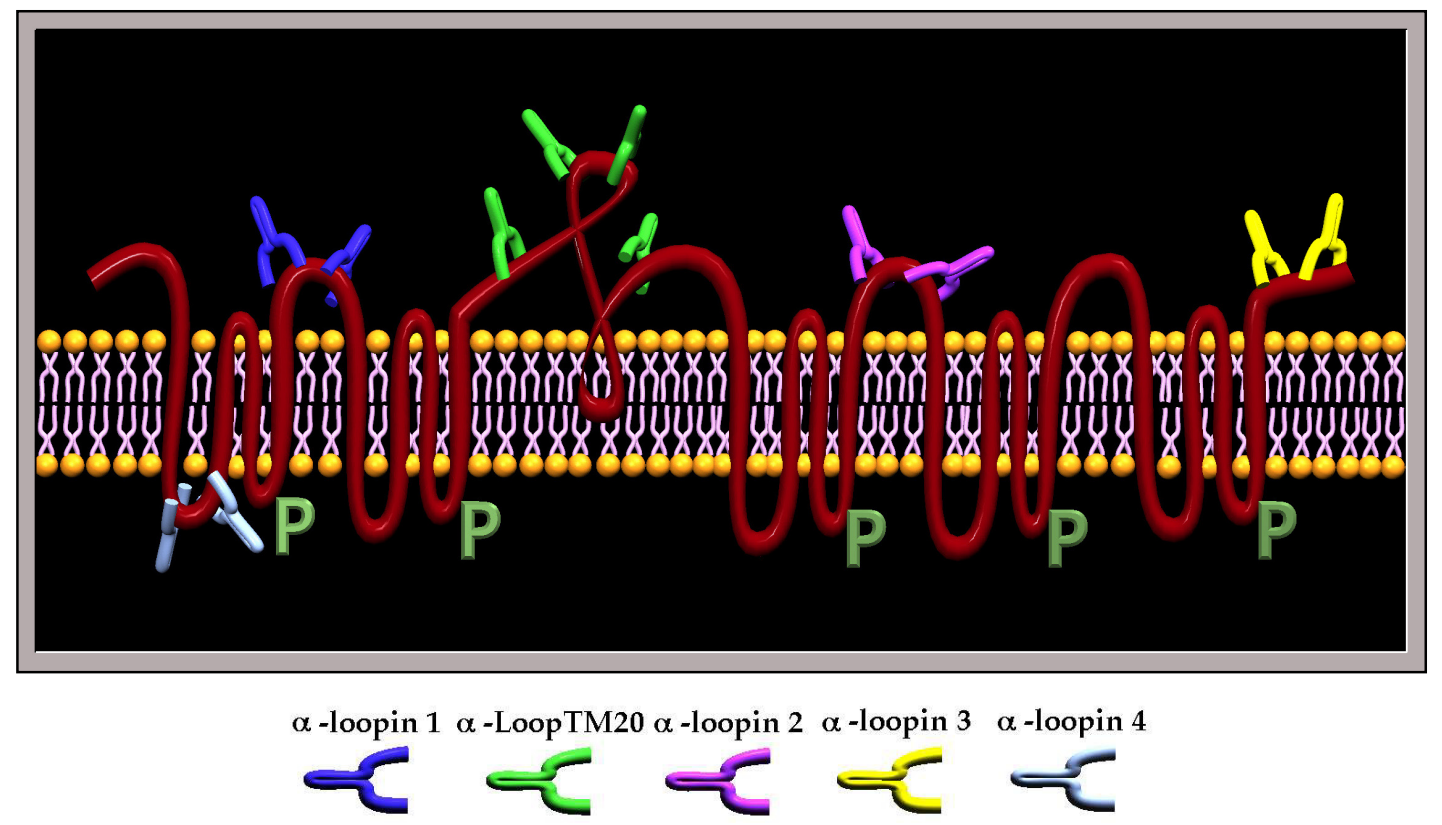

Figure 3.11: Recognition sites of the different antibodies raised against several antigenic zones ofTM20. Four of these antibodies were raised against synthesized peptides ( $\alpha$-loopin1 - $\alpha$-loopin 4$)$ and in the other case a in E.coli expressed and purified protein fragment ( $\alpha$-loopTM20) was used as antigen.

\subsubsection{Expression of the loop region in $\mathrm{pET} 28 \mathrm{a}$}

The central hydrophilic region (LoopTM20; 539aa-809aa see Fig. 1.10) was cloned into the pET28a expression vector (Novagen). This commercial expression system allows the production of recombinant proteins in Escherichia coli controlled by an IPTG inducible T7-promoter. The expressed fusion protein tagged at N-terminus with a coil of seven histidines, was purified in affinity columns containing nickel, is bound to a metal chelation resin (pET-system manual).

The correct induction of the expression of the fusion protein was checked by SDS-PAGE. After two hours of induction there was considerable accumulation of the recombinant protein compared to the non-induced protein extract (Fig 3.12 A). 
Following the purification protocol, several wash steps at different concentrations of imidazol were applied to the nickel activated affinity column to guarantee maximum purity of the eluted protein. The efficiency of the purification process was monitored by SDS-PAGE. Samples of the input, the flow through, the wash step and the final elution were loaded in a SDS-PAGE (Fig. 3.12 B).

A major part of the contaminating proteins was washed out in the wash step. Nevertheless, in the first elution with $100 \mathrm{mM}$ imidazol a significant amount of E.coli protein is detected beside the overexpressed protein. In the second elution step with $1 \mathrm{M}$ imidazole only the band of $32 \mathrm{KDa}$ and another band of $14 \mathrm{KDa}$ were observed. This latter band is probably a degradation product of the $32 \mathrm{KDa}$ protein. The solution of the first elution was passed once again through the affinity column to obtain a higher grade of purity. The final protein fragment LoopTM20 was pure enough to be used for the immunization of rabbits, with the purpose of the generation of antibodies.
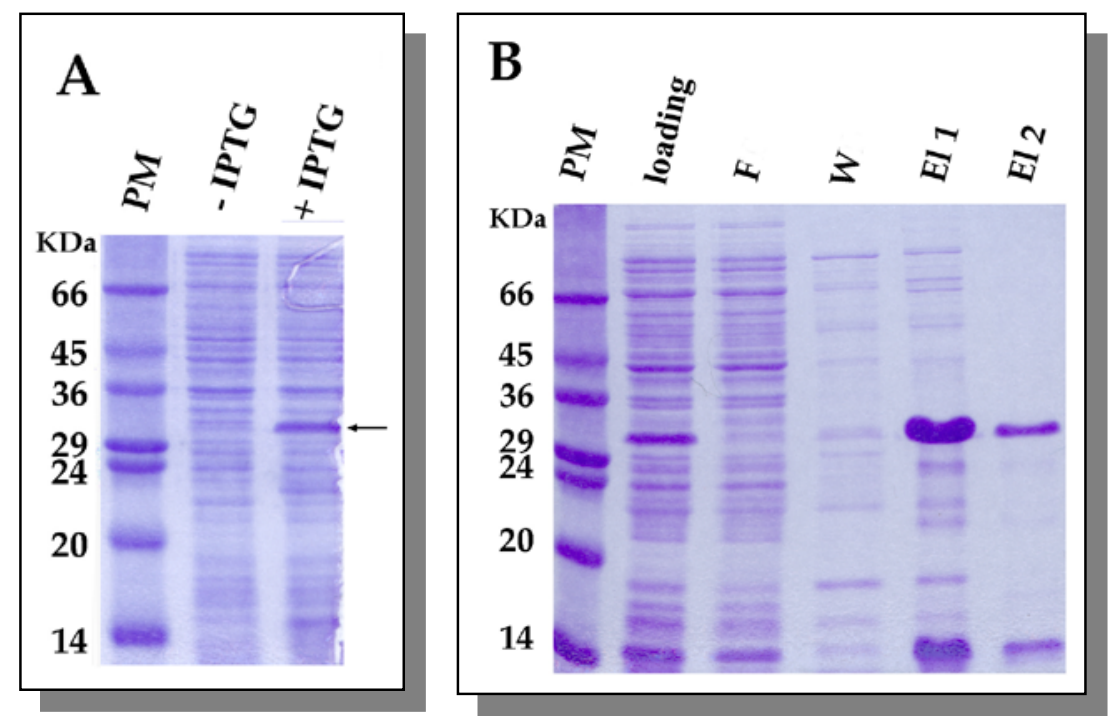

Figure 3.12: SDS-PAGE analysis of expression and purification of LoopTM20 in BL21 cells by using vector $\mathrm{pET} 28$. Coomassie-blue stained SDS-PAGE to monitor induction efficiency by IPTG. $2 \mathrm{~h}$ of induction at $37^{\circ} \mathrm{C}$ in comparison to a protein extract before the addition of IPTG. The band of $32 \mathrm{KDa}$ (see arrow) corresponds to the expected size of the recombinant protein LoopTM20. B Coomassie-blue stained SDS-PAGE to monitor the different purification steps of the overexpressed protein LoopTM20. PM - molecular weight markers; loading - protein extract with overexpressed protein loaded in the nickel activated column; F - Flow through, protein extract after passing through the purification column; W - Wash buffer, step to clean the column from non bound proteins; 20mM imidazole; El1 first elution step with $100 \mathrm{mM}$ imidazol; El2 - second elution step with 1M imidazole. The major part of the $32 \mathrm{KDa}$ protein was retained in the purification column. 


\subsubsection{Synthetic peptides}

Four different peptides of 15 amino acids each were synthesized by the "Servicio de Síntesis de Péptido, Universidad de Barcelona" (Table 3.2). Due to the small size of the antigens and to guarantee a sufficiently strong immunological response it was necessary to couple the peptides to a carrier protein (KLH) through a cysteine at the C-terminus or the $\mathrm{N}$-terminus.

All the described antigens (E.coli expressed and purified protein and peptides) were injected in rabbits for the production of antibodies. One week after each immunization step the affinity and concentration of the specific antibodies was monitored by western blot analysis in dot-blots, to detect the corresponding antigen.

\begin{tabular}{|c|c|c|}
\hline loopin 1 (329aa-342aa) & FVSEKRFVPKRLKD-C-KLH & $\alpha$-loopin 1 \\
\hline loopin 2 (1015aa-1018aa) & SQRSDADDKTWERR-C-KLH & $\alpha$-loopin 2 \\
\hline loopin 3 (1376aa-1389aa) & KLH-C-RAPTASTRIPGDHA & $\alpha$-loopin 3 \\
\hline loopin 4 (203aa-217aa) & YNAGLTPPGGFWSKN-C-KLH & $\alpha$-loopin 4 \\
\hline (389aа-403aа) & YQAGLSPPGGFWAEN & \\
\hline (831aa-845aа) & Y $\bar{Q} S G L \bar{N}$ PPGGFWTQR & \\
\hline (1034aa-1048aa) & Y $\overline{\mathrm{QA}} G I \bar{N} P P G G V W \overline{\mathrm{SDD}}$ & \\
\hline (1217aa-1231aa) & 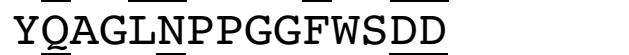 & \\
\hline
\end{tabular}

Table 3.2: List of the peptides used for the production of antibodies. The Cys residue is the link to the carrier protein KLH and also the start of synthesis of the peptides, so is found in all the listed peptides. The sequence coding for the peptide loopin 4 can be found in the entire sequence of TM20 five times with slight modifications. The amino acids differing from the sequence of loopin4 are underlined. In brackets the position of the amino acids referring to Figure 1.10.

The peptides loopin1-loopin3 were chosen for their predicted antigenicity and for their localization in the loops between two membrane domains (see Figure 3.11). In this way the probability of antigen recognition by the antibodies would be higher due to the better exposure of the antigen. The decision to raise antibodies against the peptide loopin4 was based on the homology between loopin4 and the proteins of Arabidopsis described in the previous chapter. Assuming the correct function of the 
antibody, it might be used for immunological studies in maize and also in Arabidopsis. Homologous sequences of the peptide loopin4 can be found with slight modifications in five regions repeated over the amino acid sequence of TM20. This implies, that the polyclonal antibody could recognize these regions as well.

\subsubsection{Characterisation of the antibodies}

Before the antibodies were employed for further studies it was necessary to test their affinity for the antigen and their specifity. Consequently, the different sera were tested in western blot analysis with the antigens as samples. Serum originated from non-immunized rabbits (NI) was always used as a control to the ECL signal obtained from the different antibodies.

\subsubsection{Antibodies against the recombinant LoopTM20}

In Figure 3.13 is shown the result of the detection of the recombinant protein TM20 by the antibody $\alpha$-LoopTM20. The antibody $\alpha$-LoopTM20 detected a major band with a molecular weight of about $32 \mathrm{KDa}$. This size corresponds to the expected size of the protein fragment expressed in E.coli. Among the variety of bands that were observed, only one band with a molecular weight of about $64 \mathrm{KDa}$ gave a signal comparable to the band of $32 \mathrm{KDa}$. The appearance of this signal and the background

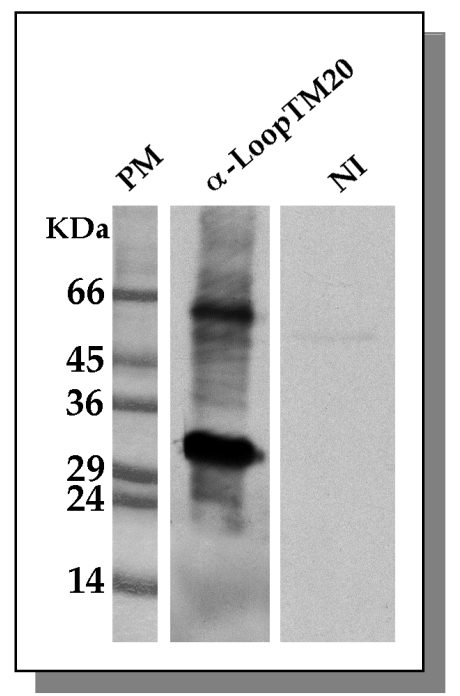

Figure 3.13 Western blot of the protein LoopTM20 using antibody $\alpha$-LoopTM20 (dilution 1:500) and ECL. As negative control the serum from nonimmunized rabbit was applied (NI). The major band at $32 \mathrm{KDa}$ corresponds to the overexpressed protein LoopTM20. 
can be explained by the possible contamination with proteins of E.coli. The same sample was incubated with pre-immune serum NI as negative control and no significant ECL signal was detected, demonstrating that the background originates only from the antibodies raised against the contaminating proteins of E.coli. Nevertheless the TM20 fragment represents the protein recognized by the serum with the highest affinity and the antibody $\alpha$-LoopTM20 was ready for use in further studies.

\subsubsection{Antibodies against the synthetic peptides}

In the case of the peptides it was not possible to resolve them by gel electrophoresis due to their small size. For this reason, to identify the specific IgGs in the serum against peptides, different concentrations of peptides were dotted directly on a PVDF membrane.

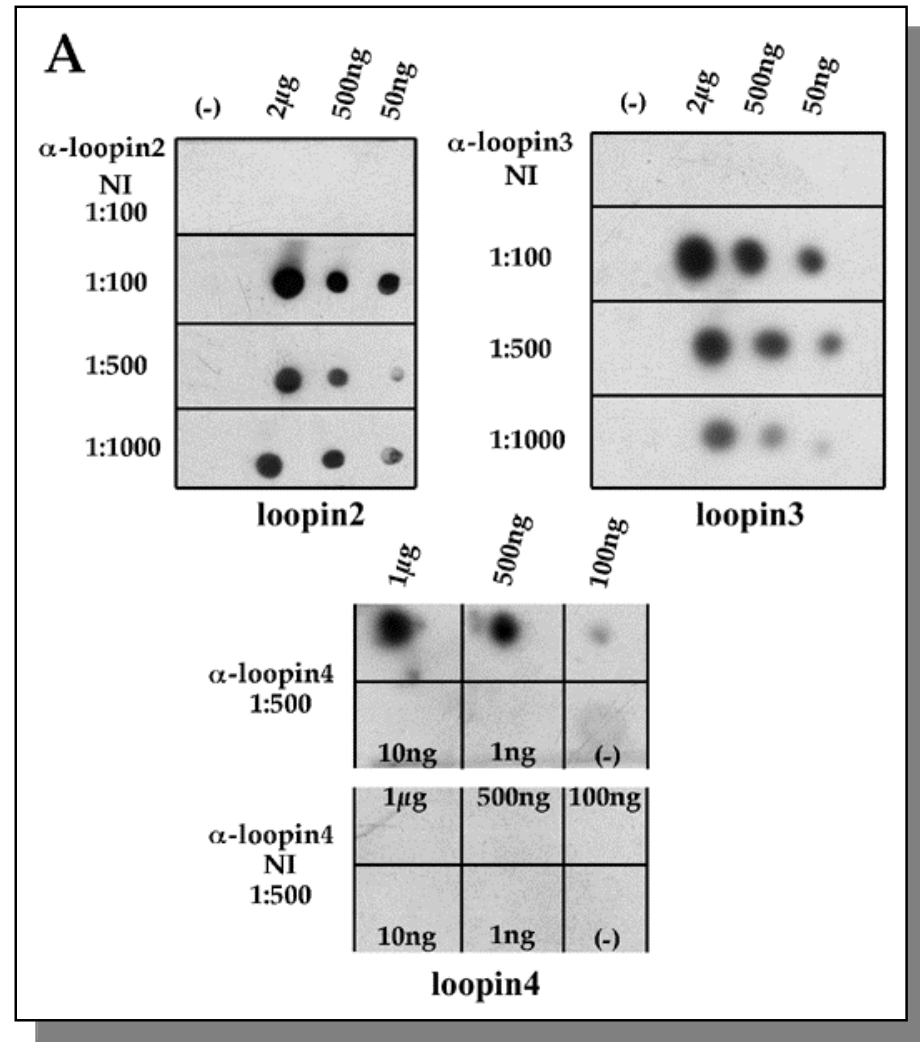

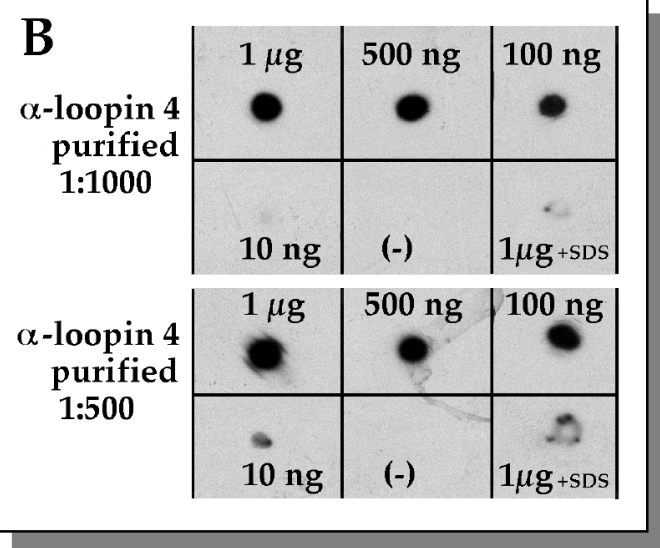

Figure 3.14: Dot-blot immuno analysis of antibodies against synthetic peptides. A Dotblots of peptides loopin2. loopin3 and loopin4 at different concentrations, incubated with the corresponding immune serum in different dilutions and with pre-immune serum NI. Negative control (-) was: $1 \mu \mathrm{g}$ of peptide loopin1. B Dot-blots after purification of $\alpha$-loopin4 antibody by immunoaffinity with SulfoLink ${ }^{\mathrm{TM}}$ columns. Different concentrations of peptide loopin4 and Ab-dilutions. Comparison of the purified $\mathrm{Ab}$ to the FT of the purification. (-) negative control represented by $1 \mu \mathrm{g}$ of peptide loopin3. 
As shown in Figure 3.14 $\mathrm{A}$ the antibodies $\alpha$-loopin2, $\alpha$-loopin3 and $\alpha$-loopin4

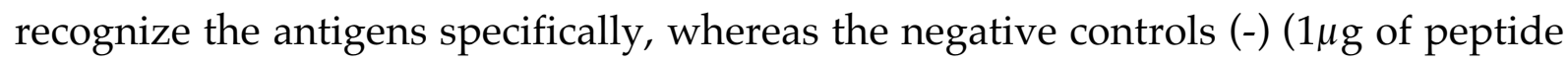
loopin1) were not detected by the antibodies. In addition, the negative control with pre-immune serum NI did not show any crossreaction.

The affinity of the antibodies was not very high, therefore immuno-affinity purification with SulfoLink ${ }^{\mathrm{TM}}$ sepharose columns (Pierce) to concentrate and purify the antigen specific IgGs was done. The reduced peptides were covalently coupled to the sulphur activated resin, and by passing the corresponding serums through the columns, the specific antibodies were retained. After elution at low $\mathrm{pH}(2.5)$ the buffer was changed and the purified antibodies tested in Dot-blots (Fig. 3.13 B). In this Dot-blot experiment we observed, that the antibody $\alpha$-loopin 4 only recognizes the peptide loopin 4 in the native state. The detection sensitivity of the peptide loopin 4 in presence of $0,5 \%$ SDS was clearly reduced. However, the antibody $\alpha-$ loopin 4 was used for in situ immunolocalization since the samples were not treated with denaturating chemicals.

The concentration and efficiency of the antibodies $\alpha$-loopin 2, $\alpha$-loopin 3 and $\alpha$-loopin 4 improved after immuno purification, as shown in Figure 3.13 A,B. In conclusion, the characterisation of the obtained antibodies confirmed their specificity and consequently they were used to detect TM20 in extracts containing low concentrations of TM20. However, the recognition efficiency of antibodies raised against small peptides is lower than against larger protein fragments expressed in E.coli. In consequence, the detection of low abundant proteins may be more complicated. On the other hand, the purity of synthetic peptides is normally higher, because of no contamination with E.coli proteins, and antibodies raised against synthetic peptides should provide a clear signal and show less crossreaction.

\subsubsection{Detection of TM20 in maize embryos}

After the establishment of their correct function, the purified antibodies were used in western blot analysis to detect TM20 in protein extractions of maize embryos of 10, 15 and 20 DAP. The northern blot analysis of TM20 showed that the mRNA of 
TM20 is most abundant in embryos up to 20 DAP (Stiefel et al. 1999). The aim of this analysis was first to test if the antibodies would recognize TM20 in protein extracts and whether they can be used for further studies of subcellular localization and immunolocalization.

\subsubsection{TM20 detection in microsomes of maize embryos at different developmental stages}

The 20 transmembrane domains of TM20 are an indication of its membrane localization. To resolve the relation of TM20 to membranes, protein extracts from maize embryos were separated into fractions of soluble proteins (S150) and microsomes (P150) by ultracentrifugation (150000g). This method separates membranes from soluble proteins and enriches membrane related proteins. In this study the membrane enriched protein fractions (P150) of maize embryos of 10 DAP, $15 \mathrm{DAP}$ and $20 \mathrm{DAP}$ (whole embryo and isolated embryo axis) were placed onto a PVDF membrane. The proteins of S150 were precipitated with TCA and were placed also onto the PVDF membrane. The results obtained using the purified antibody $\alpha-$ loopin 4 are presented in Figure 3.15 and show a clear signal in fraction P150 (1-4) as well as traces in fraction S150 (6-9). At 10DAP the signal was very low, probably due to the lower amount of total protein in the protein preparation shown by Ponceau red staining of the membrane. Since TM20 possess characteristics of a membrane integral protein, it should be found mainly in the microsomal fraction. The detection of TM20 in the fractions of the soluble proteins may be as a result of contamination of microsomal proteins during the process of membrane preparation. The negative control (Fig. 3.15B) showed no signal and confirms the specific detection of TM20 by $\alpha$-loopin4. As a negative control the membrane was incubated with the secondary antibody, a peroxidase labeled $\alpha$-rabbit antibody. This result confirmed the supposed association of TM20 to cell membranes and the ability of the antibody to detect native TM20 in protein extractions of maize embryos. Furthermore, the result documented the presence of TM20 at developmental stages in accordance with the results of the performed northern blot analyses. 
Figure 3.15: Dot-blot analysis of microsomal fractions of maize embryos of 10DAP, 15DAP, 20DAP and 20DAP embryo without scutellum. A Dot-blot incubated with $\alpha$-loopin4 (1:250) and Ponceau-red staining of the membrane. B Dot-blot incubated with the secondary antibody ( $\alpha$-rabbit) as negative control. $1-4$ microsomal fractions P150 of maize embryos: 1 - 10DAP; 2 - 15 DAP; 3 20DAP; 4 - 20 DAP only the embryo axis; 5- positive control $1 \mu \mathrm{g}$ loopin $4 ; 6$ - 9 soluble protein fractions S150 precipitated with TCA. $6-10 \mathrm{DAP} ; 7$ - 15DAP; 8 - 20DAP; 9 - 20DAP only embryo axis.

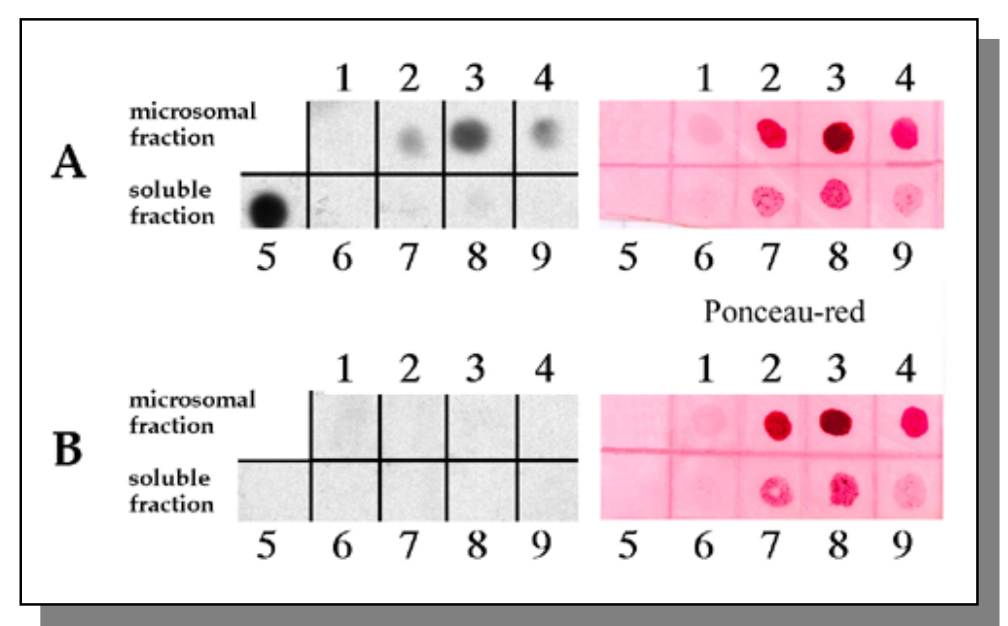

\subsubsection{SDS-PAGE and western analysis of 20DAP maize embryo microsomes}

To confirm the positive result of the Dot-blot analysis, proteins of P150 and S150 fractions of 20DAP embryos were analyzed. The proteins of both fractions were concentrated by TCA precipitation and separated by SDS-PAGE. After the transfer of the proteins to a PVDF membrane by electroblotting, the membranes were incubated with the affinity purified antibodies $\alpha$-loopin $1,-2,-3$ and -4 . Only two antibodies, $\alpha$ loopin 2 and $\alpha$-loopin 4 , showed a specific recognition (Fig. 3.16). The antibodies $\alpha$ loopin1 and $\alpha$-loopin3, even though purified, gave variety of bands (not shown) that were classified as non-specific.

The band, which appeared in the western blots, had a molecular weight of about $53 \mathrm{KDa}$ and was detected by two different antibodies. In the case of $\alpha$-loopin2 the same band could be observed in the microsomal fraction and in the cytoplasm fraction. This result had been already observed in the dot-blot analysis (see 3.2.3.1) and may due to the fact that the separation of the soluble fraction and the microsomal fraction is not total. The correct preparation of the P150 fraction and the S150 fraction were tested by western blots with two control antibodies. The antibody $\alpha$-Calreticulin specifically detects the protein calreticulin from the ER, whereas $\alpha$ ATPase recognizes an ATPase specific to the plasma membrane. The two antibodies 
detected the corresponding proteins of approximately $50 \mathrm{KDa}$ (calreticulin) and 100 KDa (ATPase) and confirmed the correct microsomal preparation.

The most striking point of these results is the small size of the detected protein. The predicted size for TM20 was $152 \mathrm{KDa}$ and the protein labeled by the antibodies $\alpha$-loopin 2 and $\alpha$-loopin 4 had a molecular weight of only a third of the expected size. This difference between the expected and the detected size may be due to degradation processes. Degradation during protein preparation is a common problem and affects mostly large proteins. On the other hand it can not be excluded that the reduced size detected for TM20 is due to posttranscriptional modifications of TM20 and that TM20 develops its activity in a smaller protein form.

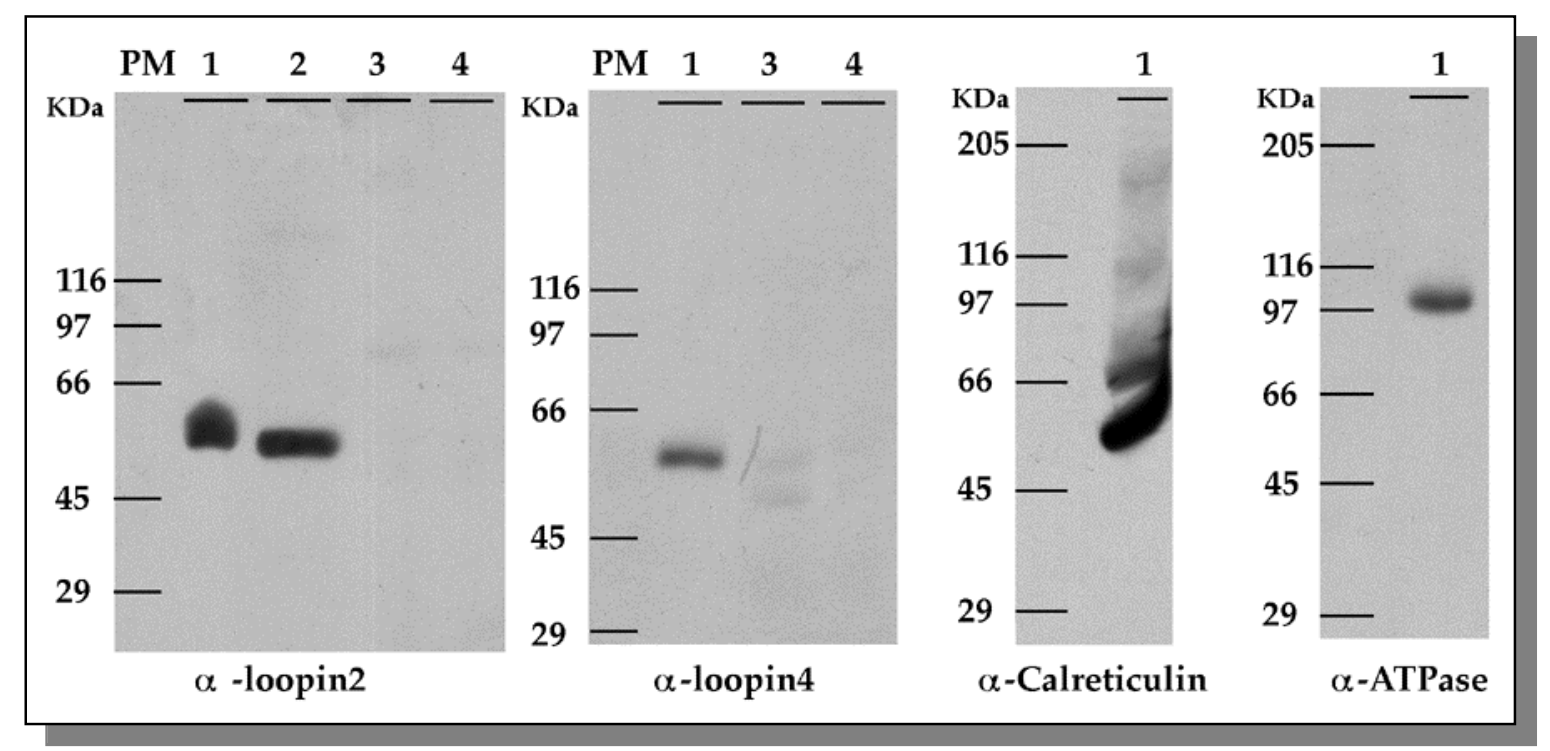

Figure 3.16: Immunodetection of TM20 in protein extraction of 20DAP maize embryos. The two antibodies $\alpha$-loopin 2 and $\alpha$-loopin 4 each detected a band at aproximately $53 \mathrm{KDa}$. As control for correct membrane preparation, two antibodies were used, marking the ER specific protein calreticulin and an antibody specific for an ATPase localized in the plasmamembrane. 1 - P150 fraction of 20DAP maize embryo; 2- S150 fraction of 20DAP old maize embryo; 3 - in vitro transcription and translation of TM20 cDNA (TNT); 4 - control reaction without DNA template (TNT). Antibody dilutions: $\alpha$-calreticulin 1:2000; $\alpha$-ATPase 1:1000; $\alpha$-loopin2 1:250; $\alpha$-loopin4 $1: 100$.

As a control and to confirm the molecular weight of TM20 labeled by the antibodies, an in vitro transcription and translation of TM20 was carried out. For this purpose the TNT ${ }^{\mathrm{TM}}$ system was used which permits transcription and translation in one reaction mix. Moreover, the reaction kit allows a radioactive labeling or non- 
radioactive of the protein. The full length TM20 cloned in pBluescript was used as template DNA. The same template was used to obtain cRNA for further injection in oocytes of Xenopus laevis, using the Promega in vitro transcription system (see 3.5.2). While the in vitro transcription with the Promega system lead to a correct transcript, no translation product of the $\mathrm{TNT}^{\mathrm{TM}}$ reaction could be obtained. The lack of any translation product may due to the complex structure of TM20 and that the in vitro TNT $^{\mathrm{TM}}$ system does not fulfil the conditions to translate the TM20 transcript correctly. The problem is probably based in the translation reaction, since the in vitro transcription system of Promega provided a correct transcript of TM20.

Similarly, the attempts to obtain a western blot of the entire protein (152KDa) of TM20 failed. As mentioned before, it is not clear if the problems are due to the size and structure of TM20, which may facilitate degradation, or if post-transcriptional modifications complicate the detection of the entire protein. Nevertheless, these problems in characterizing TM20 reflect the difficulties that complicate the study of proteins with complex structures and large size.

\subsubsection{SDS-PAGE and western analysis of maize leaves}

Since TM20 was described as an embryo specific gene (Stiefel et al. 1999) and a $53 \mathrm{KDa}$ protein was identified in protein extractions of 20DAP maize embryos, no expression would be expected in non-embryonic tissue. A separation of P150 and S150 fractions was carried out with leaves of germinated maize plants (5 DAG; days after germination). The proteins were separated by SDS-PAGE and transferred to a PVDF membrane. The results of the western blot analysis are documented in Figure 3.17. Lanes 1 and 2 loaded with the P150 fraction (1) and the S150 fraction (2) showed no signal at all. On the other hand in microsomal protein fractions (P150) of maize embryos of 26 DAP (lane 3), the $53 \mathrm{KDa}$ band could be detected, as before (see 3.2.3.2). As a control for correct protein preparation the antibodies $\alpha$-calreticuline and the $\alpha$-ATPase were employed. The $50 \mathrm{KDa}$ calreticuline was labeled mainly in P150, but significant label was also detected in S150 by the corresponding antibody (Fig. 3.17). The ATPase, which is exclusively located in the plasma membrane, was detected only in the P150 fraction. These results confirm the specific presence of 
TM20 during early stages of embryogenesis and the low abundance or absence in tissues of the germinated plant. The observed pattern of TM20 protein accumulation is in accordance with the pattern of RNA accumulation of TM20 described by Stiefel et al (1999).

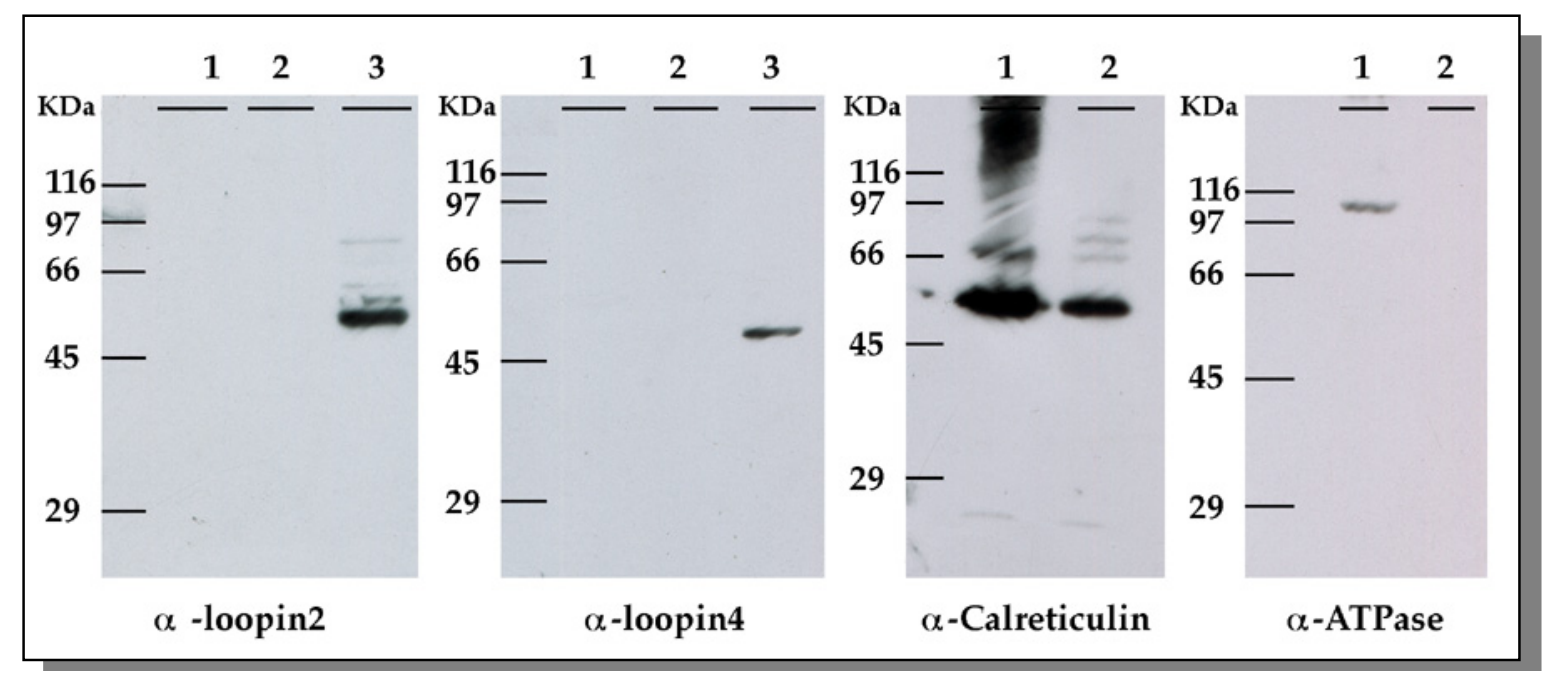

Figure 3.17: Western blot analysis of P150 and S150 fractions from 5 DAG maize leaves. Incubation was carried out with the antibodies $\alpha$-loopin 2 and $\alpha$-loopin 4 recognizing TM20. As control for the preparation of S150 and P150 membranes were incubated also with the antibodies $\alpha$-Calreticulin and $\alpha$-ATPase. 1- P150 fractions from leaf (5DAG); 2- S150 fraction (5DAG); 3 - P150 fraction from maize embryos 26DAP. Antibody dilutions: $\alpha$-calreticulin 1:2000; $\alpha$-ATPase 1:1000; $\alpha$-loopin2 1:250; $\alpha$-loopin4 1:100.

\subsection{Immunolocalization}

The technique of western blotting can be used to detect the presence in protein extractions from specific tissues and can demonstrate the presence or absence of a specific protein. However, if the exact localization of a protein in tissue or at the subcellular level is desired, the use of microscopy is necessary. To detect a protein in situ an indirect labeling method is performed. Secondary antibodies conjugated with fluorescent molecules (fluorochromes) bind to protein specific IgGs and can be visualized by fluorescence light microscopy or confocal microscopy. Depending on 
the efficiency of the protein specific antibodies it is even possible to obtain information about the location at subcellular level, although the best method for this purpose is immunogoldlabeling and the use of electron microscopy.

After the determination of the specifity of the antibodies raised against TM20, immunolocalization on tissue of 10DAP, 15DAP and 20DAP maize embryos was carried out. For the detection of TM20 in embryonic tissue an amplification system was used, employing a secondary antibody anti-rabbit conjugated with biotin. The biotin was labelled with streptavidin conjugated with a fluorochrome. In all the experiments of immunohistochemistry presented here, we use the purified $\alpha$-loopin 4 antibody.

\subsection{1 "In situ" detection of TM20 in maize embryos}

\subsubsection{Immunohistochemistry on sections of 20DAP maize embryos}

Since the northern blot had shown that TM20 is expressed in embryos up to 30 days after pollination (Stiefel et al. 1999), the first immunolocalizations were carried out with 20DAP embryos. The accumulation of TM20 transcripts at this age is maximum and on the other hand the size of an embryo of 20 DAP assures the comfortable handling during the process of sample preparation and sectioning.

For the different studies of immunolocalization streptavidine conjugated with Rhodamine-red (Jackson Immunoresearch) or Oregon-green 488 (Jackson Immunoresearch) were used. Oregon-green 488 was the most frequently used fluorochrome, since the excitation wavelength for Rhodamine-red produces a strong background in the cells of the scutellum. This background fluorescence was not observed for Oregon-green.

The Figure 3.18 shows the immunolocalization of TM20 in the longitudinal section of a whole maize embryo of 20 DAP labeled with Oregon-green 488 . The expression pattern of TM20 marked by the fluorescence is mainly found in the young foliar primordia and the apical meristem. In the part below the epicotyl, so called 
mesocotyl, two strands of cells labeled by fluorescence can be observed. The part of the embryo axis which is known as the scutellar node or first node (Esau 1960) displays only a trace of labeled cells. The labelling became more intensive in the radical. The labelling of TM20 in the root seems to be more intensive in the outer cell layers of the central vascular cylinder. Nevertheless both the apical part of the embryo and the basal part show more fluorescence than the scutellar node or the mesocotyl. In the scutellum no fluorescence could be detected. The observed pattern corresponds to the accumulation pattern of TM20 mRNA in 20DAP old embryos, with the leaf primordia and the radical as the sites of the most intensive label (Stiefel et al. 1999).

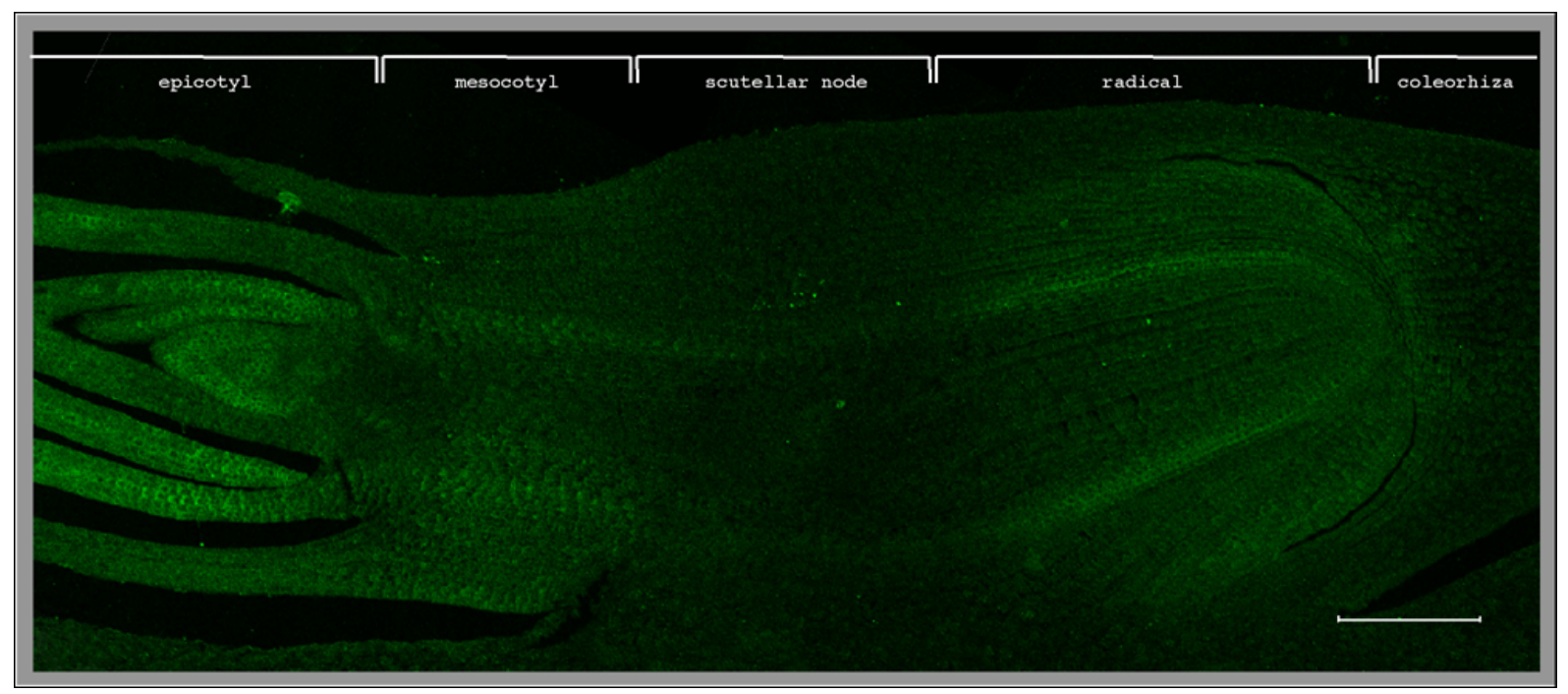

Figure 3.18: Immunolocalization of TM20 in the longitudinal section of a 20DAP maize embryo. The antibody employed was $\alpha$-loopin4 (dilution 1:100; secondary antibody: anti-rabbit conjugated with biotin and streptavidine conjugated with Oregon-green 488). The main fluorescence is located in the leaves and the meristem as well as in the root. Scale bar $=200 \mu \mathrm{m}$

The labelling pattern of TM20 displayed in Figure 3.18 was found in all the immunolocalizations of 20DAP embryos (Fig. 3.19). The fluorochrome used in this experiments was Rhodamine-red. The specificity of the antibody was confirmed by comparing the labeled tissues to sections of 20DAP embryos, which were incubated only with the secondary antibody as negative control (Fig. 3.19 J,K). This negative control revealed a strong autofluorescence of the scutellum (not shown here) in all sections using the excitation wavelength for Rhodamin-red. 


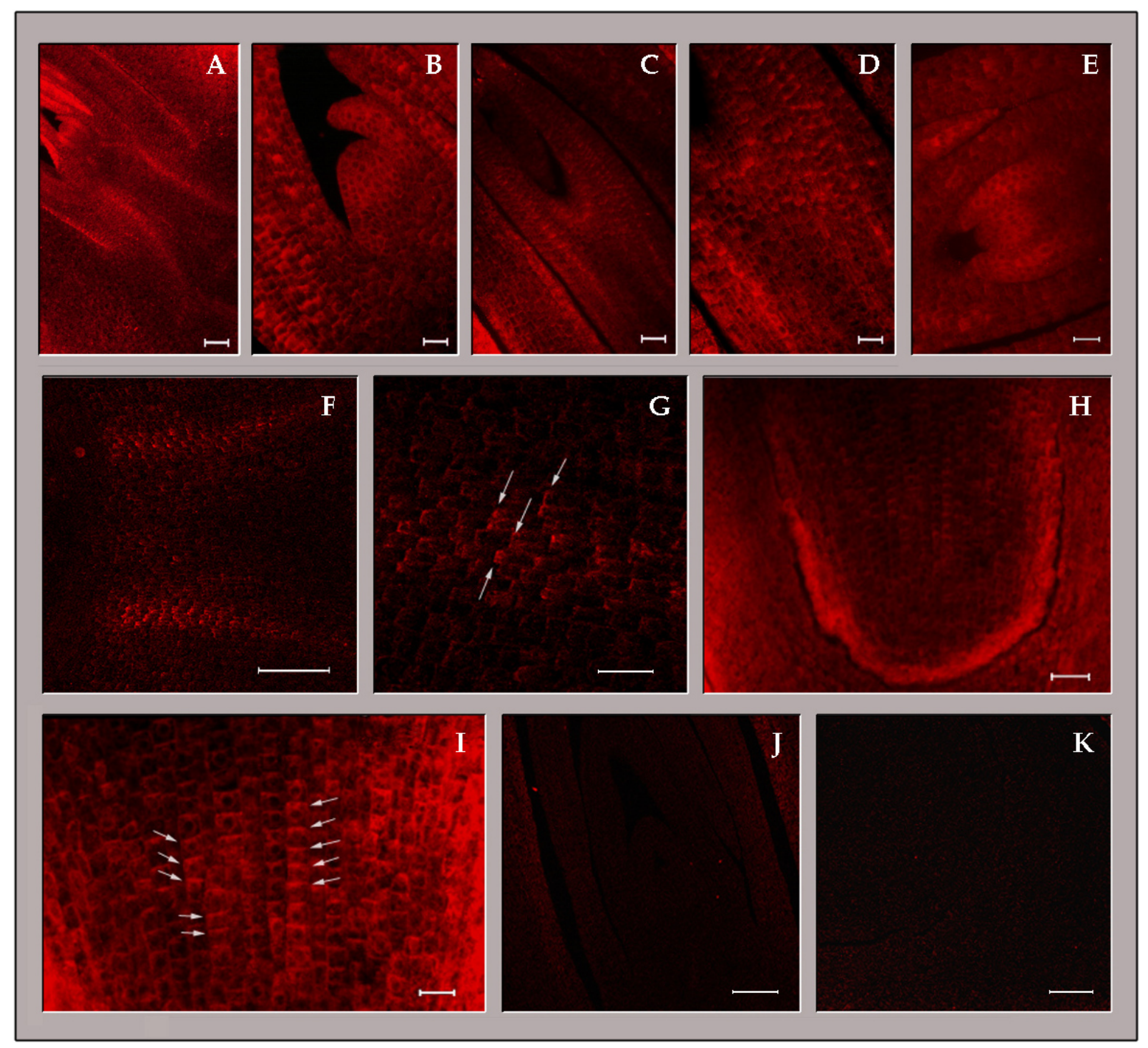

Figure 3.19: Immunolocalization of TM20 in longitudinal sections of 20DAP maize embryos. A meristem and mesocotyl; B - shoot apical meristem; C - leaf; D - leaf ; E - meristem; F - mesocotyl; G - mesocotyl; H - root tip; I - root tip; J - negative control shoot apical meristem; $\mathbf{K}$ - negative control root; Scale bars: $\mathbf{A}, \mathbf{F}, \mathbf{J}=100 \mu \mathrm{m} ; \mathbf{K}=80 \mu \mathrm{m} ; \mathbf{C}, \mathbf{H}=50 \mu \mathrm{m} ; \mathbf{B}, \mathbf{D}, \mathbf{E}, \mathbf{I}=25 \mu \mathrm{m} ; \mathbf{G}=20 \mu \mathrm{m}$.

At higher magnification it is possible to observe the label restricted to some cell layers in the leaf primordia (Fig. 3.19 A-D). In this stage of development it is still difficult to distinguish different cell types. However the indicated cells may form part of the provascular tissue due to their location. The same characterisation is valid for the cells labeled in the mesocotyl (Fig. 3.19 F,G). These strands of cells link the coleoptile with the root but also represent the connection of the embryo axis to the scutellum, necessary for the transport of nutrients. At the subcellular level, TM20 label seems to be only in the apical part of the cells. Since the label gives an effect of 
polarisation inside the cell (Fig. 3.19 G-I) no fluorescence is found on the lateral or basal cell parts. This polarisation effect was not found in the apical meristem where the TM20 label was observed inside the entire cell lumen with the exception of the nucleus (Fig. 3.19 E).

Due to the small cell size and the limited magnification by light microscopy an exact statement about the localization of TM20 inside the cell or a specific relation to cell organelles can be resolved only by electron microscopy. Nevertheless this data, together with the results of the western blots of microsomal fractions of embryonic protein extractions (see 3.2.3.2 and 3.2.3.3), indicate that TM20 seems to locate mainly in the plasma membrane.

With the aim of obtaining more information about the tissues containing TM20, immunolocalization on transversal section of 20DAP embryos were performed. In the case of a relation between the provascular tissue of the plant and the fluorescence labeled cells, immunohistochemistry over transversal sections might clarify this question. Figure $\mathbf{3 . 2 0}$ shows a series of sections of the apical shoot meristem of the 20DAP maize embryo. Clearly distinguishable are the tip of the meristem and the two meristems surrounding leaf primordia. All these organs are protected by the coleoptile. As shown in Figure 3.20 the fluorescence labeled cells are found in the middle of the leaves and in the meristem. In the first sections, the fluorescence is more dispersed in the leaves and the meristem (Fig. 3.20 A-C). In more basal sections the label becomes more restricted to special cell groups (Fig. 3.20 D-F). The labelling pattern consists of spots located in the central part of the young leaves (arrows), whereas in the meristem the signal can still be observed all over the tissue without any obviously defined pattern (Fig. 3.20 D-F).

The result of the immunolocalization on transversal sections of maize embryo is in accordance with the data of the in situ mRNA hybridization of TM20 (Stiefel et al. 1999). TM20 transcript accumulation was found in the leaf primordia, the vascular system of the coleoptile and the shoot meristem of transversal sections of 20DAP maize embryos. 


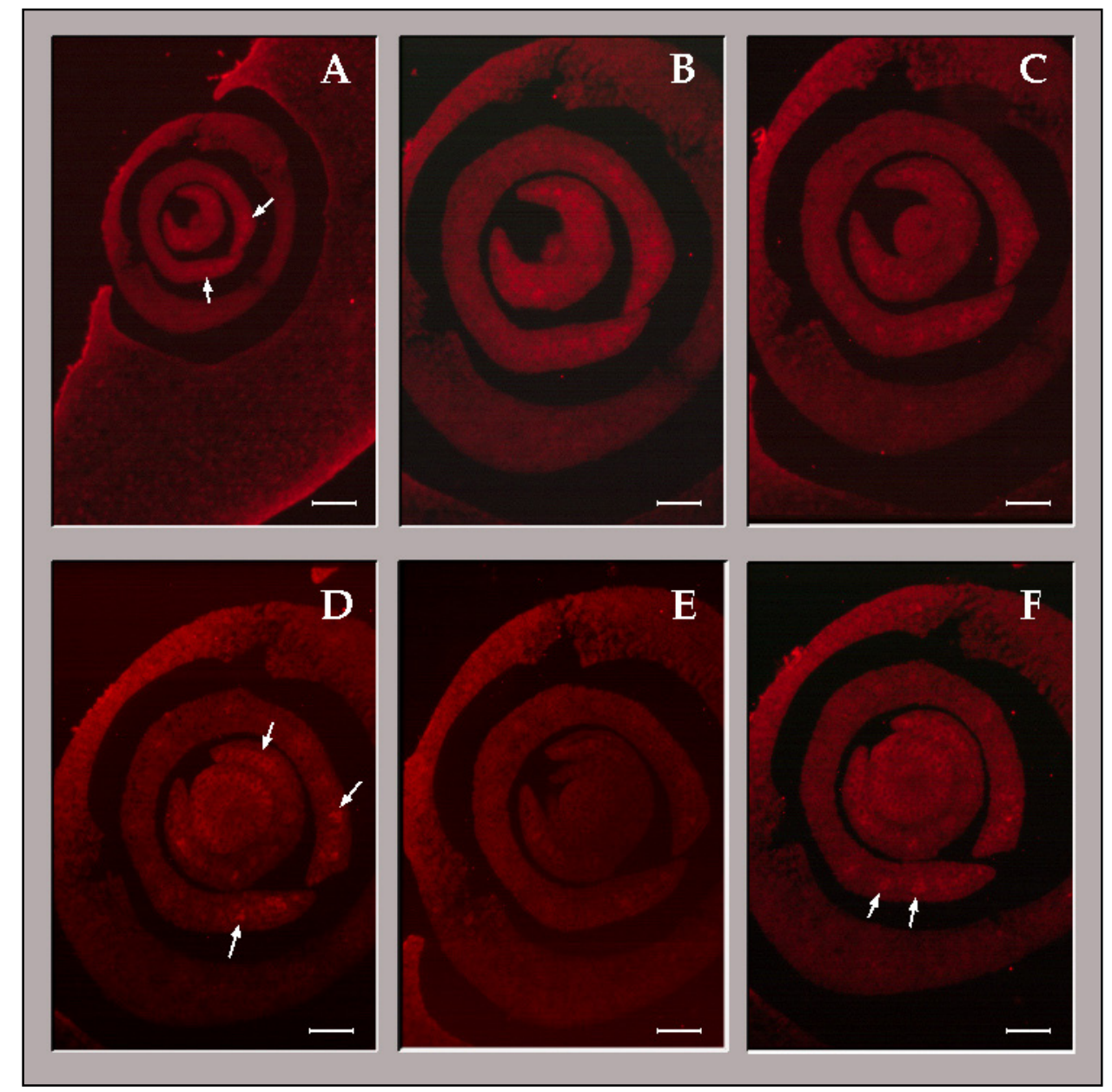

Figure 3.20: Detection of TM20 in transversal sections of 20DAP maize embryo by immunolocalization with purified $\alpha$-loopin4. Represented in the figure is a serie of transversal sections through the shoot meristem including two leaves and the coleoptile. $\mathbf{A}$ - most apical section of the shoot meristem and part of the scutellum; $\mathbf{B}-\mathbf{F}-$ section from apical to basal. Scale bars: $\mathbf{A}=200 \mu \mathrm{m} ; \mathbf{B}-\mathbf{F}=100 \mu \mathrm{m}$.

\subsubsection{Immunolocalization on 15DAP maize embryo sections}

The images shown in Figure 3.21 are the results of an immunolocalization assay on longitudinal sections of a 15 DAP maize embryo. The shoot apical meristem and the first primordia foliar surrounded by the coleoptile can be distinguished (Fig. 3.21 A). TM20 label was detected in the same zones of the 15DAP embryo as in the immunocytochemistry on 20DAP embryos. In the leaf primordia the labeled cells were located in the central layer (Fig. 3.21 A) whereas the fluorescence in the meristem was situated in the outer cell layers of the meristem (Fig. 3.21 A,B). The detection of TM20 label in the root was not as clear as in 20DAP embryos. A first observation at low magnification was the lack of a clear specific fluorescence label 
(Fig. 3.21 C). However, at higher magnification a weak label could be detected in the root at subcellular level (Fig. 3.21 D).

TM20 label is observed in the shoot meristem and the root of 15DAP embryos and shows the polarisation at the subcellular level described before for 20DAP embryos (Fig. 3.21 B,D). In contrast, in the mesocotyl no fluorescence could be detected, which may be due to the developmental stage of the embryo. This result suggests that the presence of TM20 in certain tissues is necessary at later stages of embryo development.

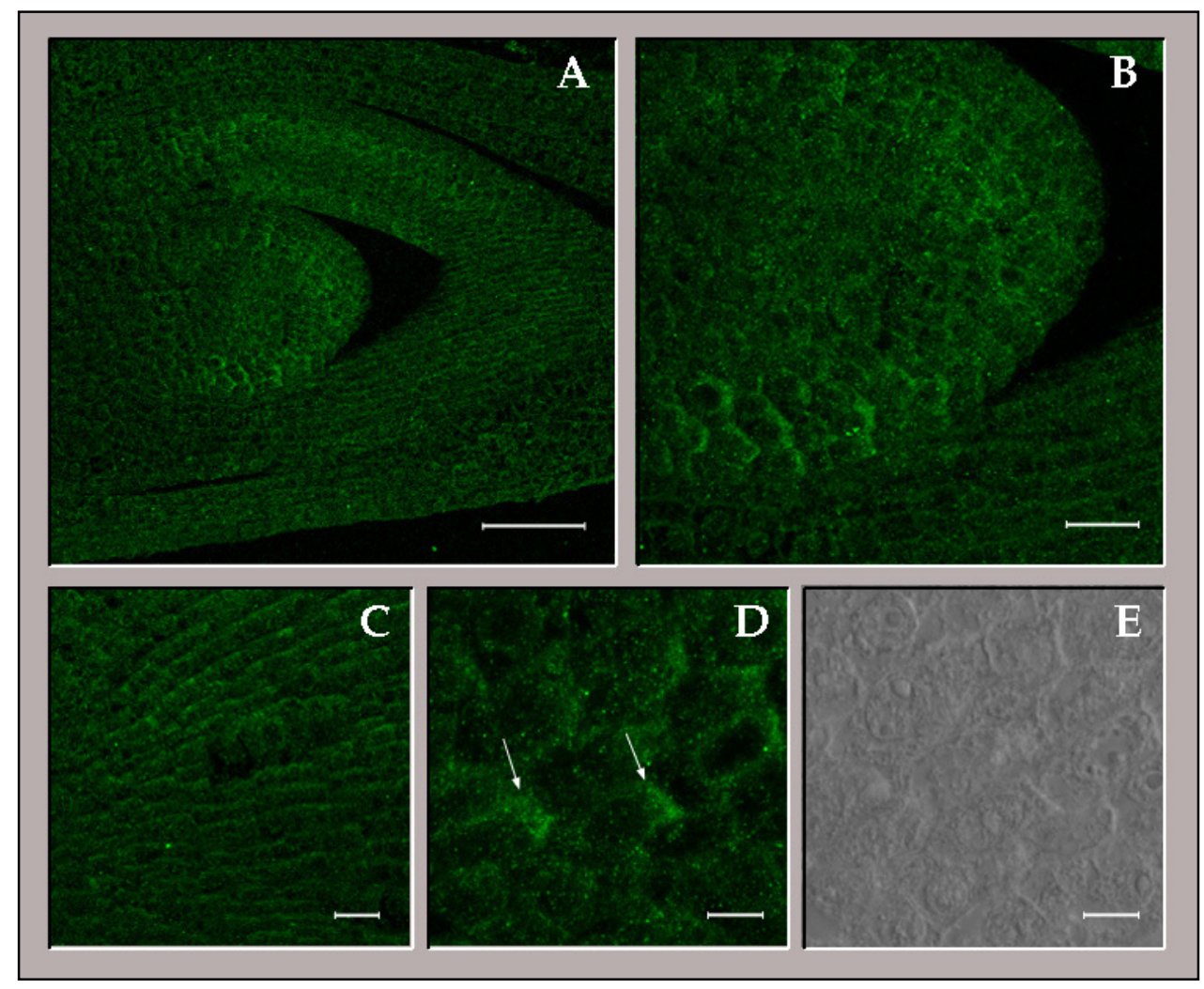

Figure 3.21: Immunolocalization of TM20 in longitudinal sections of 15DAP old maize embryo. A and B: images of the shoot meristem, showing the first leaf and the coleoptile. C root tip in low magnification; D detail of the cells of the root tip, the label is in the apical part of the cells (arrows); $\mathbf{E}$ transmission image of the same cells as in $\mathbf{D}$ to observe the cell structure. Scale bar: $\mathbf{A}=80 \mu \mathrm{m} ; \mathbf{B}$ and $\mathbf{C}$ $=20 \mu \mathrm{m} ; \mathbf{D}$ and $\mathbf{E}=8 \mu \mathrm{m}$.

\subsubsection{Whole mount immunolocalization on 10DAP maize embryos}

To detect the pattern of TM20 in embryos a few days after the establishment of the bilateral symmetry, immunohistochemistry on 10DAP embryos was performed. 
Due to the small size of the 10DAP embryos, we carried out the immunolocalization by using whole mount immunolocalization, taking advantage of the virtual sectioning of confocal microscopy (Fig. 3.22 A). Thus dehydration, embedding and sectioning of samples were avoided.

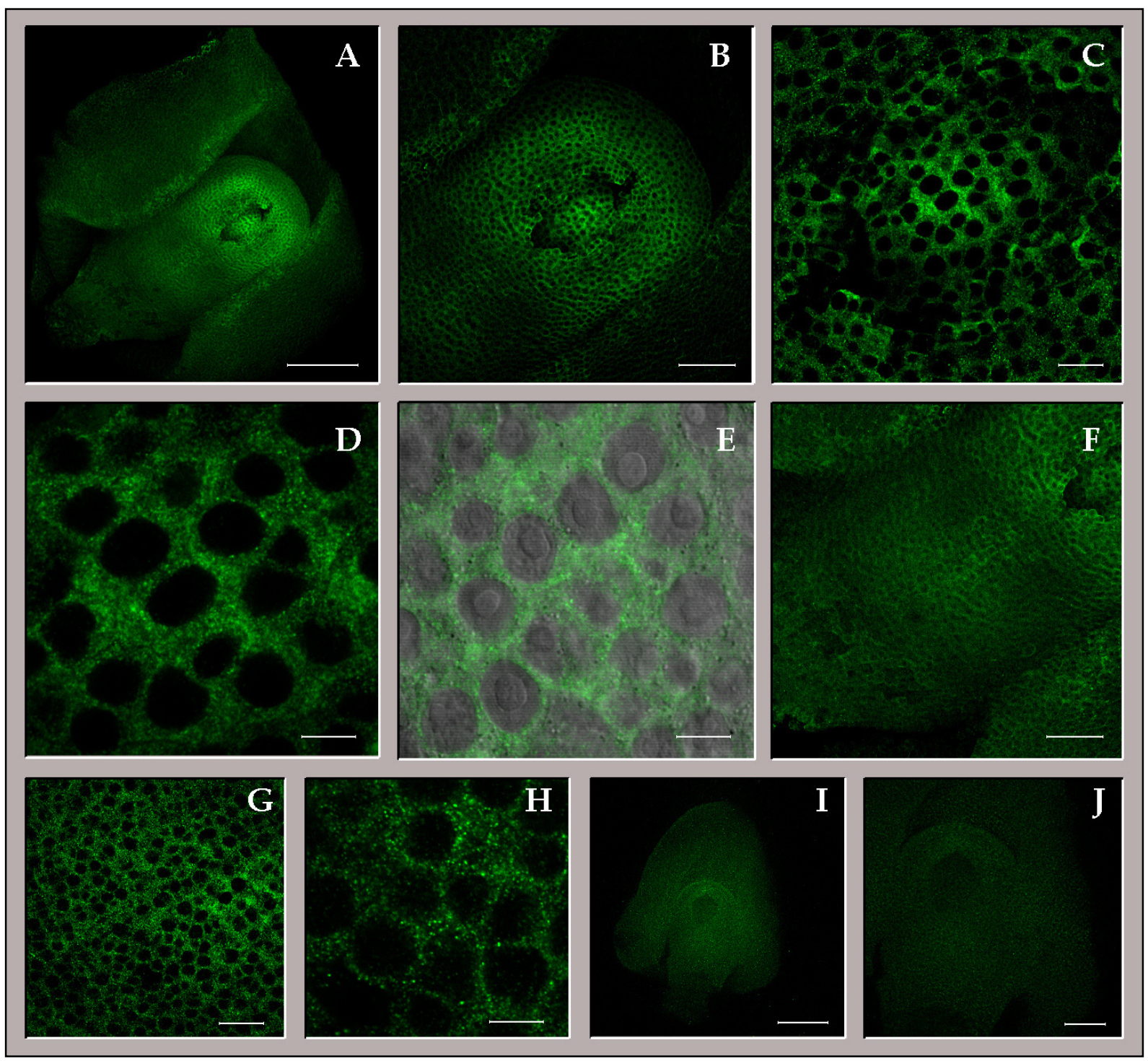

Figure 3.22 Whole mount immunohistochemistry on 10DAP maize embryos. A 10 DAP maize embryo, clearly distinguishable are the embryo axis, the rest of the suspensor and the scutellum; B Shoot apical meristem (in the centre) surrounded by the coleoptile; $\mathbf{C}$ Higher magnification of the shoot apical meristem; D Detail of cells of the shoot apical meristem; E Overlay of cells from the shoot apical meristem:transmission image. Clearly to see is the fluorescence spread all over the cell except inside the cell nucleus. Inside the nucleus good to observe the nucleolus; $F$ Basal part of the embryo, the embryo root; G Basal part at higher magnification; $\mathbf{H}$ Detail image of the root cells; I Negative control incubated only with the secondary antibody; J Negative control at higher magnification. Scale bars: $\mathbf{A}, \mathbf{I}=200 \mu \mathrm{m} ; \mathbf{B}, \mathbf{F}, \mathbf{J}=80 \mu \mathrm{m} ; \mathbf{C}, \mathbf{G}=20 \mu \mathrm{m} ; \mathbf{D}, \mathbf{E}, \mathbf{H}=8 \mu \mathrm{m}$. 


\section{Results}

As in the 15DAP and 20 DAP embryos TM20 label was detected in the apical part of the 10DAP embryo (Fig. 3.22 A-C). Strong fluorescence was observed in the central layers of the coleoptile and in the shoot apical meristem. The shoot meristem appears in the middle of the coleoptile and sticks out of the surrounding coleoptile tissue. In the basal part of the embryo axis (Fig. 3.22 F,G,H) there was slight labelling, pointing out the presence of TM20 in the early root.

The characteristic polarisation of the TM20 label pattern at the subcellular level was not observed in embryos at this developmental stage. Instead of a polarized distribution, the protein was present in nearly the whole cell except in the cell nucleus (Figure 3.22 E). This may indicate, as mentioned in the previous section, that the presence and the localization of TM20 may be dependent on the developmental stage of the embryo.

\subsubsection{Immunolocalization on lachrima mutants}

The lachrima mutant is characterized by a developmental block of the embryo in the transition stage. At this stage the embryo is clubshaped and can be distinguished as the embryo proper and suspensor. As a control for the specificity of $\alpha$-loopin4 and the observed TM20 labelling pattern, immunohistochemistry was performed with sections of paraffin embedded lachrima kernels containing the mutant embryo. Since the mutation completely inactivates the expression of the TM20 gene by an Ac-element insertion in the promoter region, no TM20 labelling was expected in lachrima embryos. The result of the immunohistochemistry shows the absence of TM20 label in the mutant embryo (Fig. 3.23). No significant fluorescence could be observed nor a difference between lachrima embryos incubated with $\alpha$-loopin4 and the negative control (Fig. 3.23 C). The absence of any label indicates that the fluorescence pattern obtained for TM20 in wild type embryos is specific. Moreover, this result is in agreement with the data obtained from northern blot analysis of lachrima mutants, where no mRNA accumulation of TM20 could be detected (Stiefel et al. 1999). 


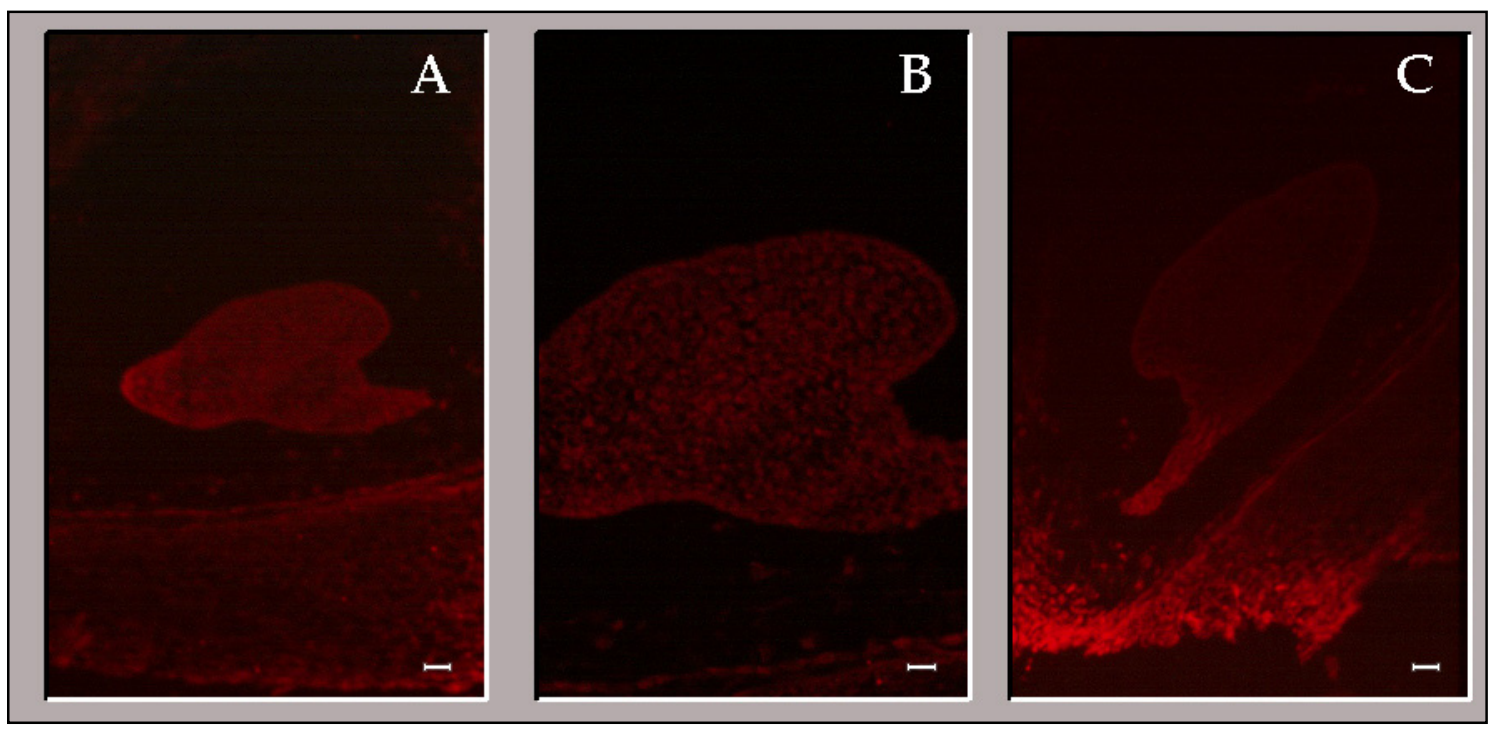

Figure 3.23: Immunohistochemistry on sections of the mutant embryo lachrima. The antibody applied in this assay was purified $\alpha$-loopin4. Due to the reduced size of the mutant embryo it was necessary to section the entire mutant kernel. A and B: Mutant embryo lachrima in different magnifications. No fluorescence could be detected in this samples; C Negative control: incubated only with the secondary antibodies. Scale bars: $\mathbf{A}, \mathbf{C}=50 \mu \mathrm{m} ; \mathbf{B}=25 \mu \mathrm{m}$.

\subsubsection{Detection of $a^{+} \mathrm{H}$-ATPase in 10DAP maize embryos}

To compare the observed labelling-pattern of TM20 in 10DAP embryos to that of a known plasma membrane located protein, monoclonal mouse antibodies $\alpha$ ATPase (Villalba et al. 1991) were used. The same antibody was used before in western blot analysis (see 3.2.3.2). Whole mount immunolocalization was carried out in 10DAP embryos and satisfactorily detected the ATPase, in the scutellum as well as in the embryo axis. A higher magnification showed the difference in the labellingpattern of the scutellar cells and embryo axis cells (Fig. 3.24). In the scutellum cells the label of the ATPase is restricted to the plasma membrane (Fig. 3.24 C,D). Every cell can be distinguished and a wavy form of the scutellum cells is well traced by the red fluorescence emitted by Cyanine 3 .

The TM20 label in cells of the embryo axis had a quite different aspect in comparison to the scutellum cells (Fig. 3.24 A,B). As observed before in the immunolocalization of TM20 in the same material (Fig. 3.22), the cells forming the 
embryo axis showed a pattern of the ATPase in the entire cell except in the nucleus. This similarity of the labelling-pattern of TM20 and the ATPase may be evidence for similar processing and trafficking of these two proteins. It can not be excluded that the stage of differentiation of the cells also plays a role in the localization of the ATPase as seen before for TM20.

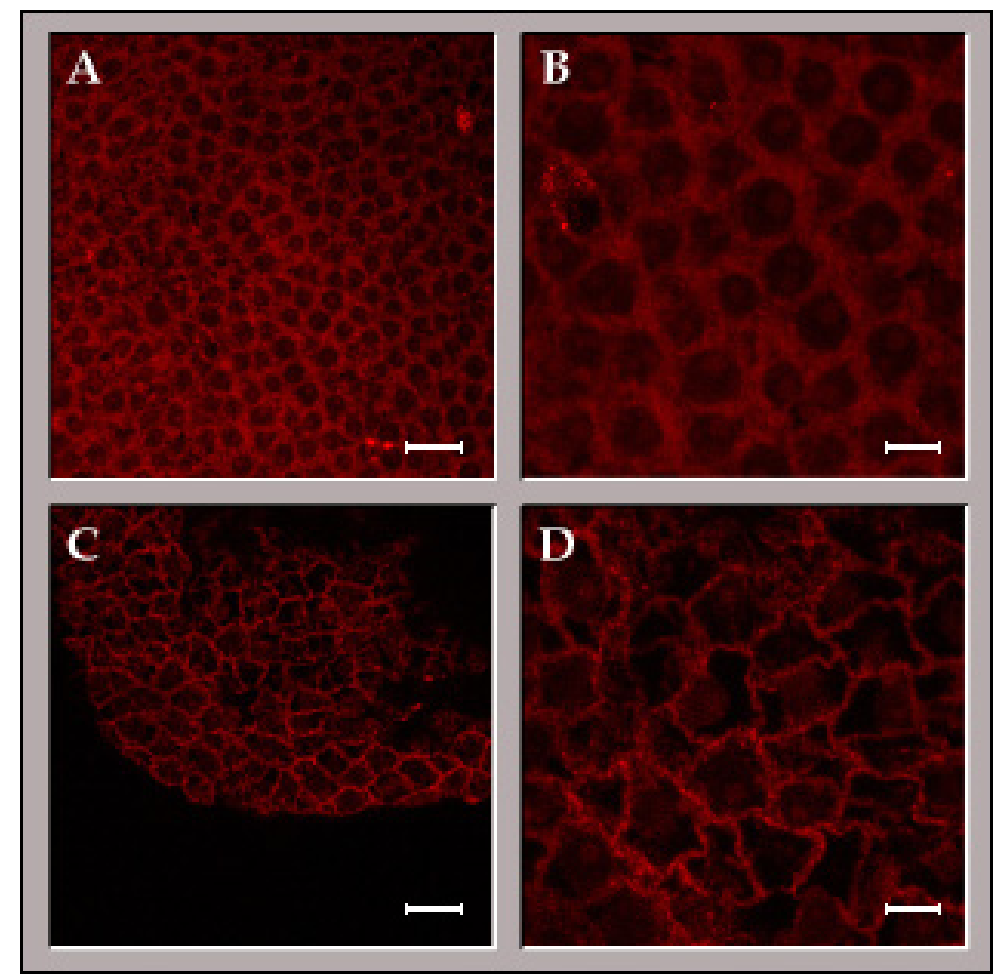

Figure 3.24: Whole mount immunohistochemistry on 10DAP maize embryo detecting a plasma membrane located ATPase. The employed antibody was $\alpha$-ATPase (1:1000). A and B image of embryo axis cells at different magnifications. $\mathbf{C}$ and $\mathbf{D}$ image of scutellum cells. The red fluorescence was observed in the plasma membrane redrawing the shape of the scutellum cells. Scale bars: $\mathbf{A}$ and $\mathbf{C}=$ $20 \mu \mathrm{m}, \mathbf{B}$ and $\mathbf{D}=8 \mu \mathrm{m}$.

\subsubsection{Detection of TM20 in Black mexican sweet (BMS) cultured cells}

The BMS cells used in this assay were derived from in vitro cultured callus cells from the Zea mays species Black mexican sweet. By changing the culture conditions it is possible to discriminate biotic or abiotic factors, which may influence the expression or suppression of specific proteins. In this study immunohistochemistry was used to determine whether TM20 is expressed in specific 
patterns in cultured BMS cells and if this model could be used for further studies to find factors important for the establishment of this pattern.

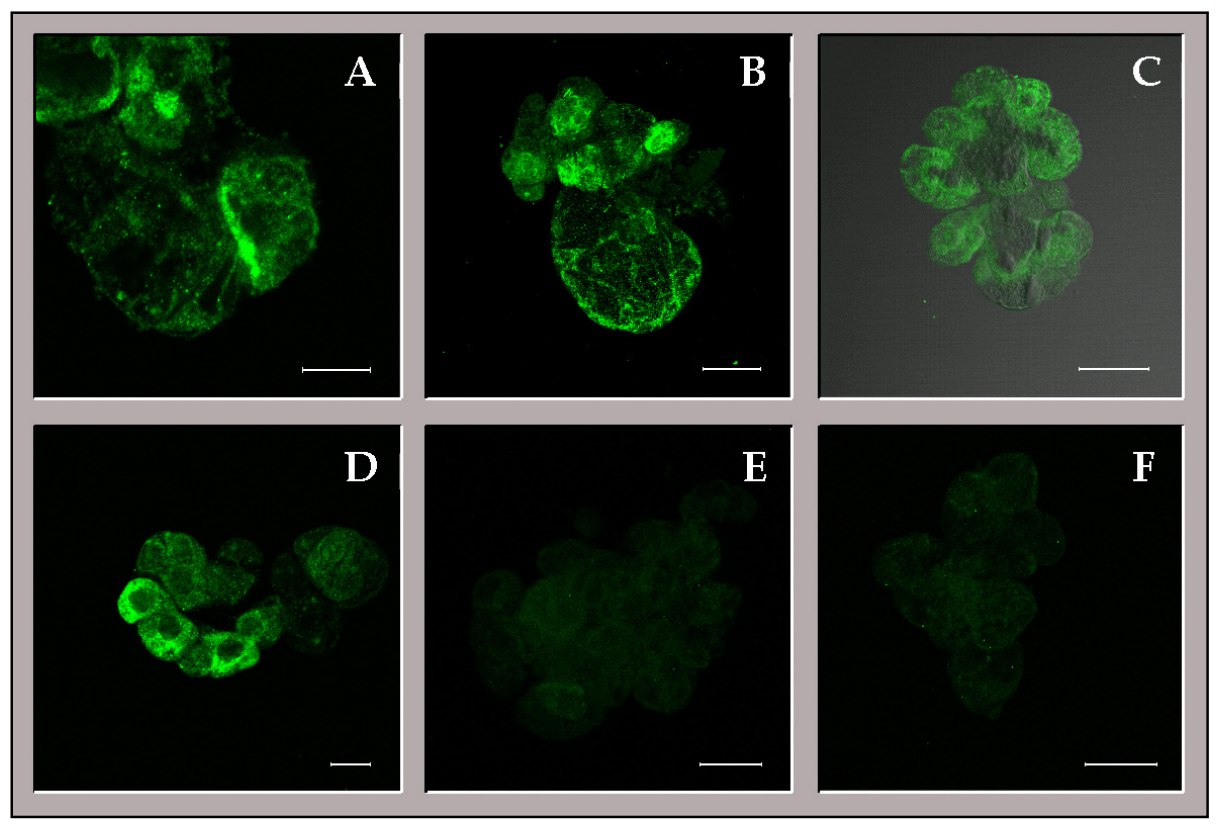

Figure 3.25: Immunolocalization of TM20 in cultured BMS cells. Different cell shapes were found in the cell cultures. The fluorescence label by antibody $\alpha$-loopin 4 was distinct for the different cell shapes. A - D examples of different cells showing different label-patterns of TM20; $\mathbf{E}$ and $\mathbf{F}$ negative control: incubated only with the secondary antibodies. Scale bars: $\mathbf{A}=16 \mu \mathrm{m} ; \mathbf{B}=80 \mu \mathrm{m} ; \mathbf{C}, \mathbf{E}, \mathbf{F}=40 \mu \mathrm{m}$; $\mathbf{D}=20 \mu \mathrm{m}$.

Immunolocalization with BMS cells could also be helpful to clarify the localization of TM20 at the subcellular level and if these cells show the same polarized labelling-pattern as observed before (see 3.3.1.1 and 3.3.1.2) in cells of embryo tissue.

The culture contained a variety of cells of different shape and the immunohistochemistry showed different fluorescence patterns (Fig. 3.25). Whereas in one kind of cells the fluorescence was observed in a spotlike manner (Fig. 3.25 A,B), the label observed in cells of smaller size was brighter and spread all over the cell (Fig. 3.25 C,D). No specific fluorescence pattern could be distinguished, although the negative controls (Fig. 3.25 E,F), incubated only with the secondary antibody, confirmed the specificity of the antibody $\alpha$-loopin4. Due to the variety of patterns no further experiments were performed with this type of cells, but the fluorescence label 


\section{Results}

indicates that TM20 is also present in these cells and their undifferentiated state may be the reason that no polarized pattern can be observed.

\subsubsection{The presence of IAA did not modify the detection pattern of TM20 in 20DAP embryos}

As mentioned before (see introduction 1.4) the involvement of TM20 in the process of polar auxin transport is discussed as a possible function of TM20. This hypothesis is based on the phenotype of the TM20 defective mutant lachrima and the structure of the protein TM20. Several studies have shown that the excess of

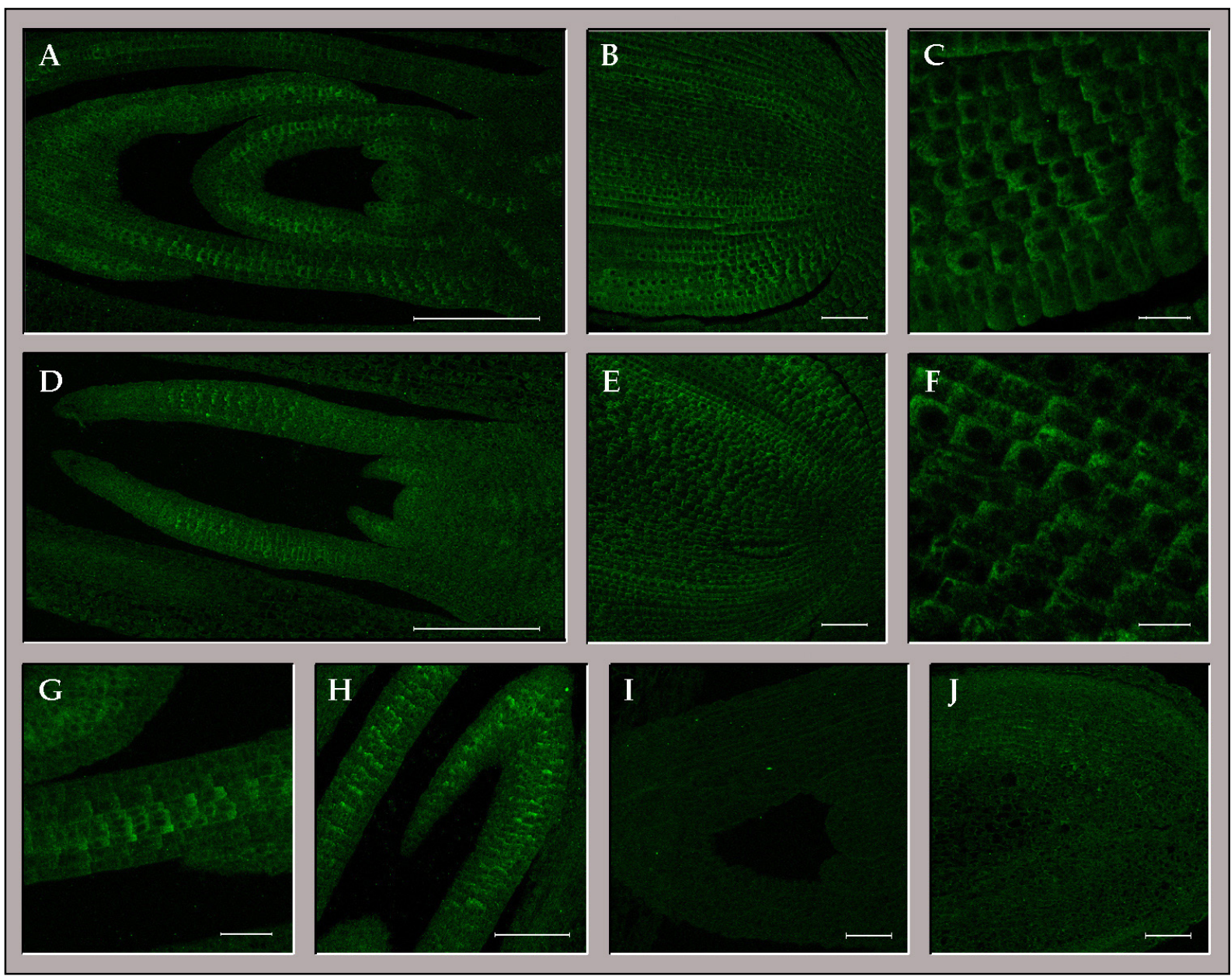

Figure 3.26: Immunolocalization of TM20 in 20DAP maize embryos cultured in the presence of additional IAA. A embryo shoot meristem cultured without exogenous IAA. See the expression pattern of TM20 in the leaf primordia and the meristem as described before (see 3.3.1.1); B embryo root tip $C$ detail image of the cells from the root tip and the cell polarisation pattern in the absence of IAA; D image of the shoot meristem of an embryo incubated with $3 \mathrm{mM}$ IAA, with the elongated leaves a good proof for the auxin action; $\mathbf{E}$ root tip from an embryo cultured in the presence of $3 \mathrm{mM}$ IAA; $\mathbf{F}$ detail image of root cells showing the polarised expression pattern of TM20; G detail image of the embryonic leaf of the embryo shown in $\mathbf{A} ; \mathbf{H}$ leaf of an embryo incubated with 3mM IAA exhibiting cells with polarised TM20 label; I and J negative control in embryo shoot meristem and embryo root: incubated only with secondary antibodies; Scale bars: $\mathbf{A}, \mathbf{D}=200 \mu \mathrm{m} ; \mathbf{B}, \mathbf{E}, \mathbf{J}=80 \mu \mathrm{m} ; \mathbf{H}, \mathbf{I}$ $=50 \mu \mathrm{m} ; \mathrm{G}=40 \mu \mathrm{m} ; \mathrm{C}=20 \mu \mathrm{m} ; \mathbf{F}=16 \mu \mathrm{m}$. 
exogenous auxin has a strong influence on developing embryos producing phenotypes similar to lachrima phenotypes (Fischer and Neuhaus 1996; Hadfi et al. 1998). This effect is produced by the exogenous auxin and is due to the disturbance of the auxin gradient in the embryo. To test if exogenous auxin interferes with the localization of TM20, 20DAP embryos were isolated from the kernel and incubated for $24 \mathrm{~h}$ on agar plates containing $3 \mathrm{mM}$ IAA and without additional IAA. After $24 \mathrm{~h}$ the samples were prepared for immunohistochemistry by fixation, embedding and sectioning.

The Figure 3.26 shows sections of 20DAP embryos incubated on agar plates without additional IAA (Fig. 3.26 A-C,G) and in the presence of 3mM IAA (Fig. 3.26 D-F,H). The elongated leaf primordia reflect the effect of IAA in the culture media (Fig. 3.26 D) as the initiation of cell elongation is a known effect of auxin on plant cells. Some of the embryos even germinated prematurely due to the presence of IAA (not shown).

No significant change of the label-pattern of TM20 between the IAA affected embryos and the embryos without IAA treatment could be observed. The pattern of polarized cells in the leaf primordia was found in the embryos not exposed to auxin action as well as in the embryos grown in the presence of auxin. In both cases the label was limited to the central cell layers of the young leaf primordia (Fig. 3.26 A,D). This observation was confirmed at higher magnification (Fig. 3.26 G,H). The same result is obtained from the immunolocalizations of TM20 in the embryo root (Fig. 3.26 B,E). The presence of IAA seems not to alter the fluorescence pattern of TM20 and at the subcellular level no significant difference could be observed (Fig. 3.26 C,F). In both cases the polarized localization of TM20 label was maintained. Sections of 20DAP embryos were incubated in parallel only with the secondary antibodies to determine possible background fluorescence (Fig. 3.26 I,J). The lack of any effect of IAA on the label pattern of TM20 indicates that TM20 localization is not influenced by IAA. On the other hand, it can not be excluded that the incubation time of $24 \mathrm{~h}$ was not sufficient to produce any significant, visible, change in the fluorescence pattern and that IAA under different experimental conditions may alter the localization of TM20. 


\subsection{Expression of a TM20:GFP construction in transfected onion epidermis cells}

With the aim of obtaining more information about the localization of TM20 at the subcellular level the N-terminus of TM20, including the N-terminus located signal peptide (Stiefel et al. 1999) and 8 transmembrane spanning domains (Fig. 3.27), was fused to GFP. This construction was used for transfection of epidermal onion cells employing the shoot-gun-method.

\subsubsection{Construction of the TM20:GFP fusion protein}

A 5' located fragment of $1640 \mathrm{bp}$ of TM20 (1 bp-1640 bp, see 1.4) was amplified by PCR and cloned into the GFP-vector pCK GFP S65C. This vector is characterized by a modified and improved GFP enabled for use in plant cells (Reichel et al. 1996). The vector was provided with a $2 \times 35 S$ promoter and with an additional translation enhancing element (TL). Since only one cloning target (NcoI) was available, the following cloning strategy was employed.

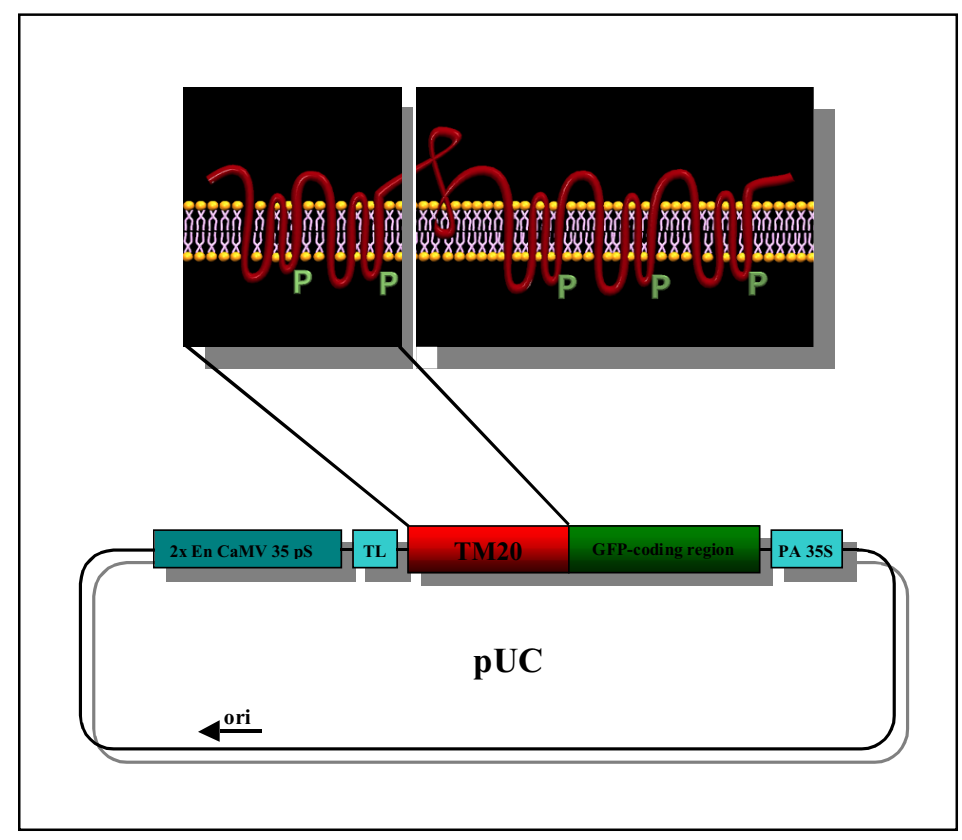

Figure 3.27: Schematic representation showing the part of TM20 cloned in the GFP-vector. The Nterminal and two groups of the transmembrane spanning domains were fused to the GFP. By automatic sequencing the conservation of the correct open reading frame was confirmed. The vector was equipped with a $2 \times 35 \mathrm{pS}$ CaMV promoter and with a translation improving sequence (TL). 
The PCR amplification of the TM20 fragment was performed with oligonucleotides starting at the $5^{\prime}$ end, including the translation start codon ATG. By the use of the Klenow fragment and T4 Polynucleotide Kinase from the Sure clone ${ }^{\mathrm{TM}}$ ligation kit (Pharmacia) the overhanging T-bases were cut off from the PCR product and the blunt ends were phosphorylated. The vector was cut by restriction enzyme digestion with NcoI and refilled using the Klenow fragment. To avoid re-ligation the blunt ends of the vector were dephosphorylated with alkaline phosphatase. After the ligation of vector and insert the constructions were sequenced to confirm the correct reading frame and to detect any PCR provoked changes in the sequence.

\subsubsection{Transfection of epidermal onion cells with TM20:GFP}

Gold bullets of $1 \mu \mathrm{m}$ diameter were covered with the DNA pCK TM20:GFP and were employed to transfect onion epidermis cells using the BioRad shot gun equipment. The transfected cells were incubated for $24 \mathrm{~h}$ at $28^{\circ} \mathrm{C}$ in the dark. The transfection result was visualized by confocal microscopy.

Figure 3.28 shows a selection of cells obtained by shotgun transfection. The green fluorescence of the GFP is clearly distinguishable in comparison with the nontransfected neighbour cells.

Examining the transfected cells in detail, the main part of the fluorescence seems to be located in the cell membrane and around the nucleus. There is no evidence for the location of the fusion protein inside the nucleus. In all observed cells the signal was never found in the vacuole, which fills nearly the whole cell lumen. However, it is not possible to discard the presence of TM20 in the cytoplasm or vesicles. Due to the extended size of the vacuole of these cells, the cytoplasm is squashed against the cell wall and it was difficult to determine if the fluorescence was restricted exclusively to the plasma membrane. On the other hand it would not be unexpected to find TM20 partially in the cytoplasm due to the intracellular trafficking processes. The data obtained from these experiments did not totally resolve the question of the final localization of TM20 due to the large vacuole of this cell type, which complicates the discrimination between plasma membrane and 


\section{Results}

cytoplasm. Although in some cells a tendency for polarization of TM20 could be observed it is not decisive.
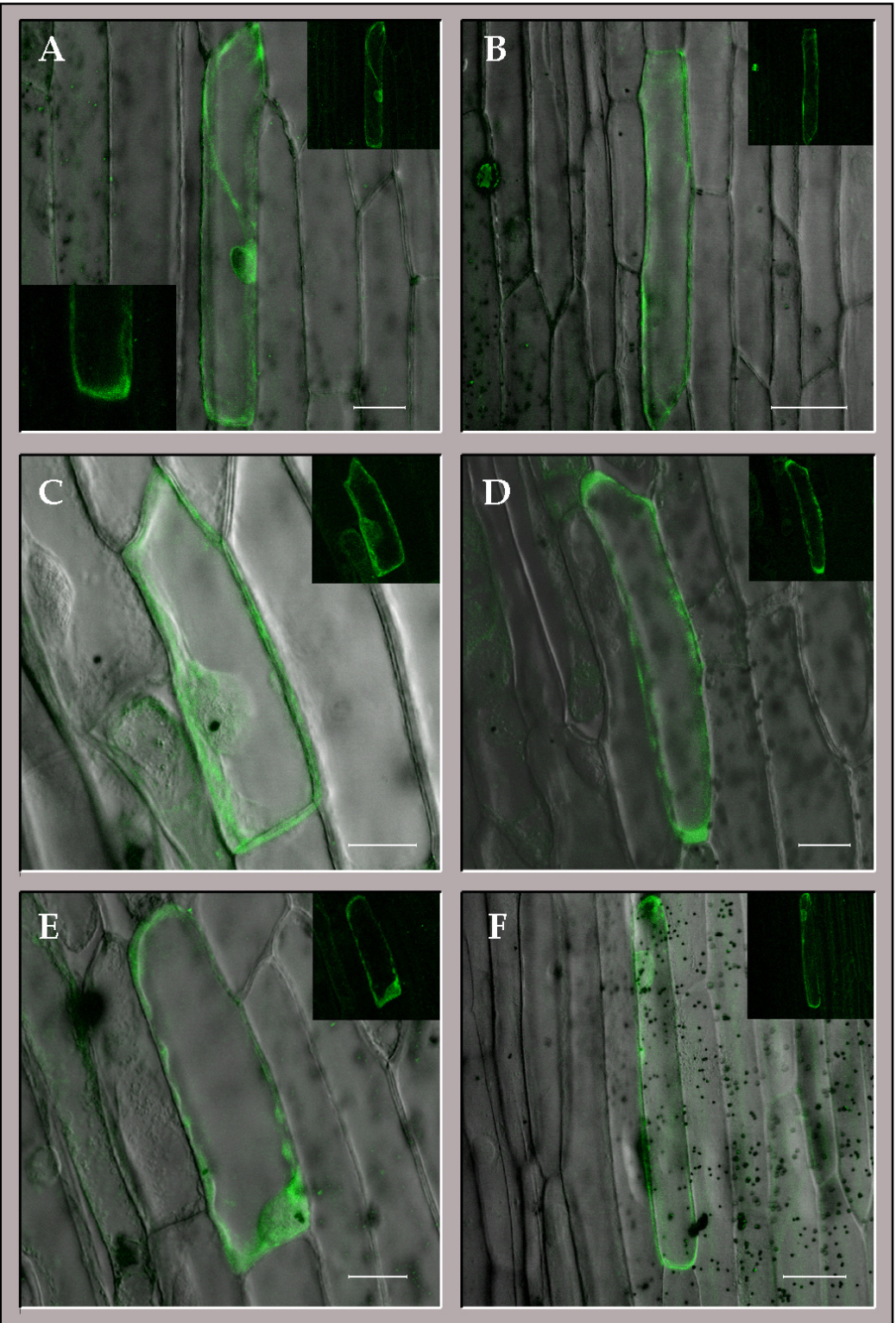

Figure 3.28: Transfection of epidermis cells of onion with the fusion protein TM20:GFP. A-F Overlay of the green fluorescence with the corresponding transmission image. The fluorescence image is displayed in the inset of every picture. The fluorescence was detected in the outer part of the cells, but not in the nucleus. Scale bars: $\mathbf{A}, \mathbf{C}, \mathbf{D}, \mathbf{E}=20 \mu \mathrm{m} ; \mathbf{B}=40 \mu \mathrm{m} ; \mathbf{F}=80 \mu \mathrm{m}$.

\subsubsection{Transfection of onion epidermis cells with pCK GFP S65C and pKAR6:GFP}

To compare the result of the TM20:GFP transfection to other fluorescence patterns, onion epidermis cells were transfected with pCK GFP S65C and with pKAR6:GFP a KDEL containing a marker protein for ER. The comparison is a control 
for the specifity of the localization observed for TM20:GFP. Figure 3.29 presents examples of the fluorescence pattern obtained by transfection of onion epidermis cells with GFP (Fig. 3.29 A,B) and with pKAR6 (Fig. 3.29 C,D).

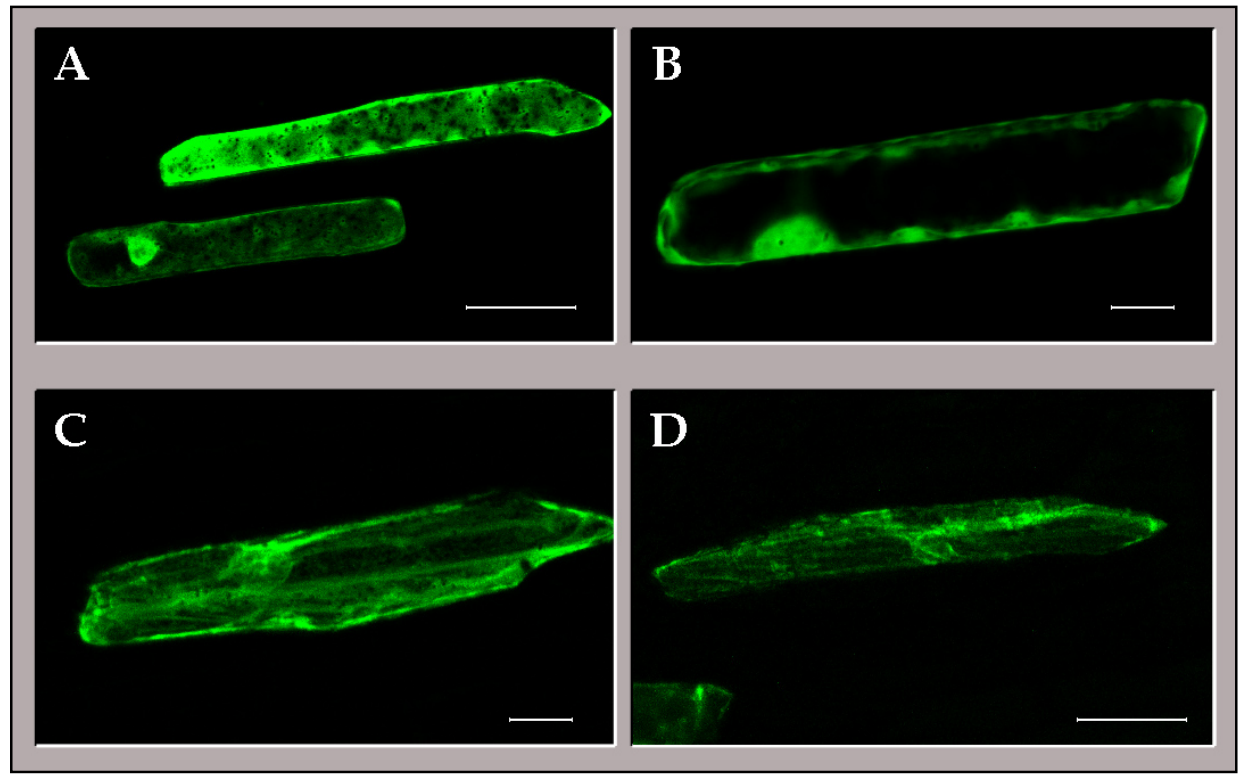

Figure 3.29: Transfection of epidermis cells from onion with pCK GFP S65C and pKAR6:GFP. A and B onion cells transfected with the green fluorescence protein. $\mathbf{C}$ and $\mathbf{D}$ transfection with pKAR6:GFP fusion protein. The fluorescence is distributed all over the cell marking the ER. Scale bars: A,D = $80 \mu \mathrm{m} ; \mathbf{B}, \mathbf{C}=20 \mu \mathrm{m}$.

The green fluorescence of the GFP was observed as expected in the nucleus and in the cytoplasm. The black spots inside the cell (Fig. 3.29 A) are cell components where the GFP could not enter and therefore they stand out black against the surrounding fluorescence. In the case that only some sections of the middle part of the cell are captured by confocal microscopy (Fig. 3.28 B) the fluorescence is restricted to the border of the cell and the nucleus. This fact has to be considered in the interpretation of the images and confirms that there are problems with this cell model in resolving protein localization in the plasma membrane.

The image offered by the cells transfected with the pCARP:GFP was different from the fluorescence pattern of GFP transfected cells. The ER label appears a red and covers the cell. This fluorescent pattern is not the same as observed for the cells transfected with TM20:GFP. However, slight fluorescence in the ER of TM20:GFP transfected cells is not unexpected due to post-translational modifications and 
intracellular trafficking. The integration of membrane located proteins takes place in general by an integration into the membrane of the ER and subsequent trafficking to the cell compartment destination (Singer 1990).

\subsection{Microinjection in oocytes of Xenopus laevis}

In addition to the identification of putative homologues and the immunological characterisation of TM20, a physiological approach to study the possible function of TM20 was chosen. As mentioned before TM20 may be related to polar auxin transport (see 1.4 and 3.3.4). To demonstrate that TM20 could be involved in polar auxin transport working as a transport protein, RNA of TM20 was microinjected in oocytes of Xenopus laevis. For this purpose the entire coding sequence of the TM20 gene was cloned into a transcription vector and was transcribed in vitro. TM20 injected oocytes were used for transport assays with radiolabeled indole-acetic-acid ( $\left.{ }^{3} \mathrm{H}-\mathrm{IAA}\right)$ (see 3.5.2).

\subsubsection{Cloning of $T M 20$ in the transcription vector pBluescript}

The first attempts to amplify two parts of the gene TM20 by PCR strategy failed, probably due to the size of the gene $(4.4 \mathrm{~kb})$. Thus the cloning strategy was changed. The sequencing of the gene provided us with many partial clones, which were analyzed and cloned through differential enzyme digestions. Figure 3.30 shows schematically the clones employed for the cloning of the full length TM20. We chose the pBluescript vector for the transcription procedures, since the partial clones were cloned in this vector. Moreover this vector allowed the start of transcription from both sides, so that the final direction of the cloned gene was not a handicap at the moment of transcription. 


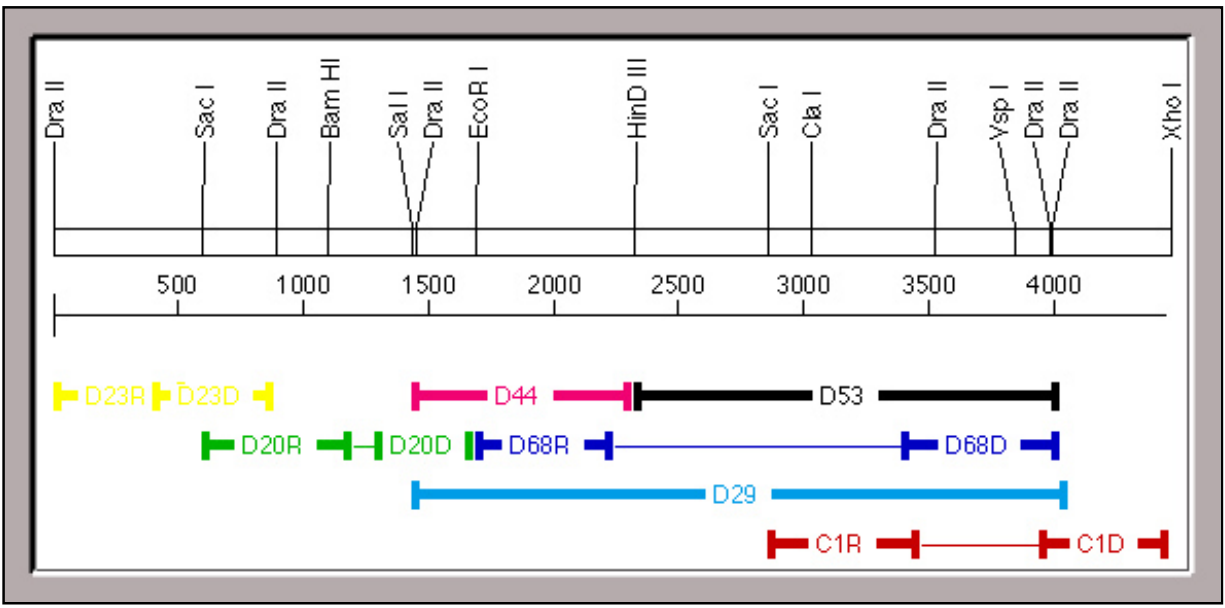

Figure 3.30: Schematic overview over the partial clones available from the screen for the full length of tm 20 covering the entire sequence. The subclones finally used for the cloning of tm 20 full length were D23,D20,D68 and C1.

The two clones D23 and D20 were joined together, and parallel the clones D68 and $\mathrm{C} 1$ were cut by digestion with restriction enzymes and joined together by ligation reactions with T4-ligase. In the final step the ligation of the two subclones lead to the full length clone of TM20. This full length clone of TM20 was subsequently used for in vitro transcription.

\subsection{2 in vitrotranscription of $T M 20$}

To obtain cRNA to inject in the oocytes, an in vitro transcription was performed. Because of the cloning direction of TM20 in the pBluescript vector, it was necessary to use the T3 polymerase (Promega) for correct transcription. The transcription protocol was modified to take into account the size (4.4 kb) of TM20. The correct size of the transcript was controlled in a denaturating agarose gel containing formaldehyde (Fig. 3.31).

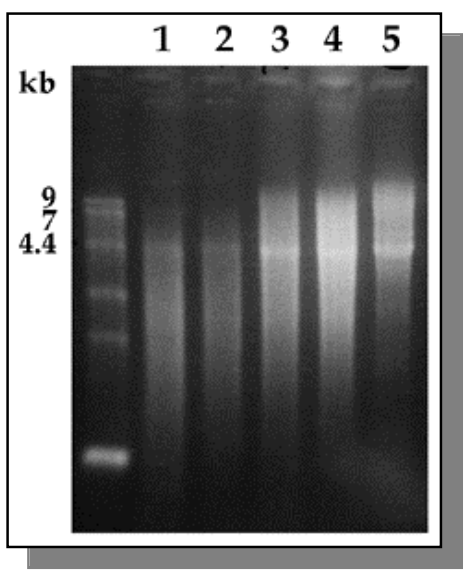

Figure 3.31: Denaturating agarose gel. The band at $4.4 \mathrm{~kb}$ corresponds to the expected size for the $t m 20$ transcript. Different transcription systems were used to test for the optimal transcription conditions. 1 - Promega Kit at $30^{\circ} \mathrm{C} ; 2-$ Promega Kit at $37^{\circ} \mathrm{C} ; 3-$ Promega Kit at $37^{\circ} \mathrm{C}$, double volume of T3-polymerase; $4-\mathrm{Kit}$ BioRad at $37^{\circ} \mathrm{C}$; 5 - Kit BioRad at $37^{\circ} \mathrm{C}$, double volume of T3-polymerase 


\section{Results}

\subsubsection{Microinjection of cRNA of TM20 in oocytes and immunocytochemistry on oocyte sections}

Oocytes were injected with cRNA of TM20 (10ng/oocyte) and incubated for three days at $18^{\circ} \mathrm{C}$. The correct localization of TM20 in the plasma membrane was tested by immunocytochemistry using the affinity purified antibody $\alpha$-loopin 4 .

\subsubsection{Immunolocalization of TM20 in oocytes}

Since the TM20 is a protein found in plant and had to be expressed in an animal system, it was necessary to confirm the correct insertion into the membrane of the oocyte. For that purpose TM20 injected oocytes and water injected oocytes were sectioned using a cryomicrotome and immunolocalization was carried out. The oocyte sections were incubated with the purified antibody $\alpha$-loopin 4 , which had been used in the immunolocalization in maize embryos (see previous chapters). An anti-rabbit antibody conjugated with biotin was used as secondary antibody and was

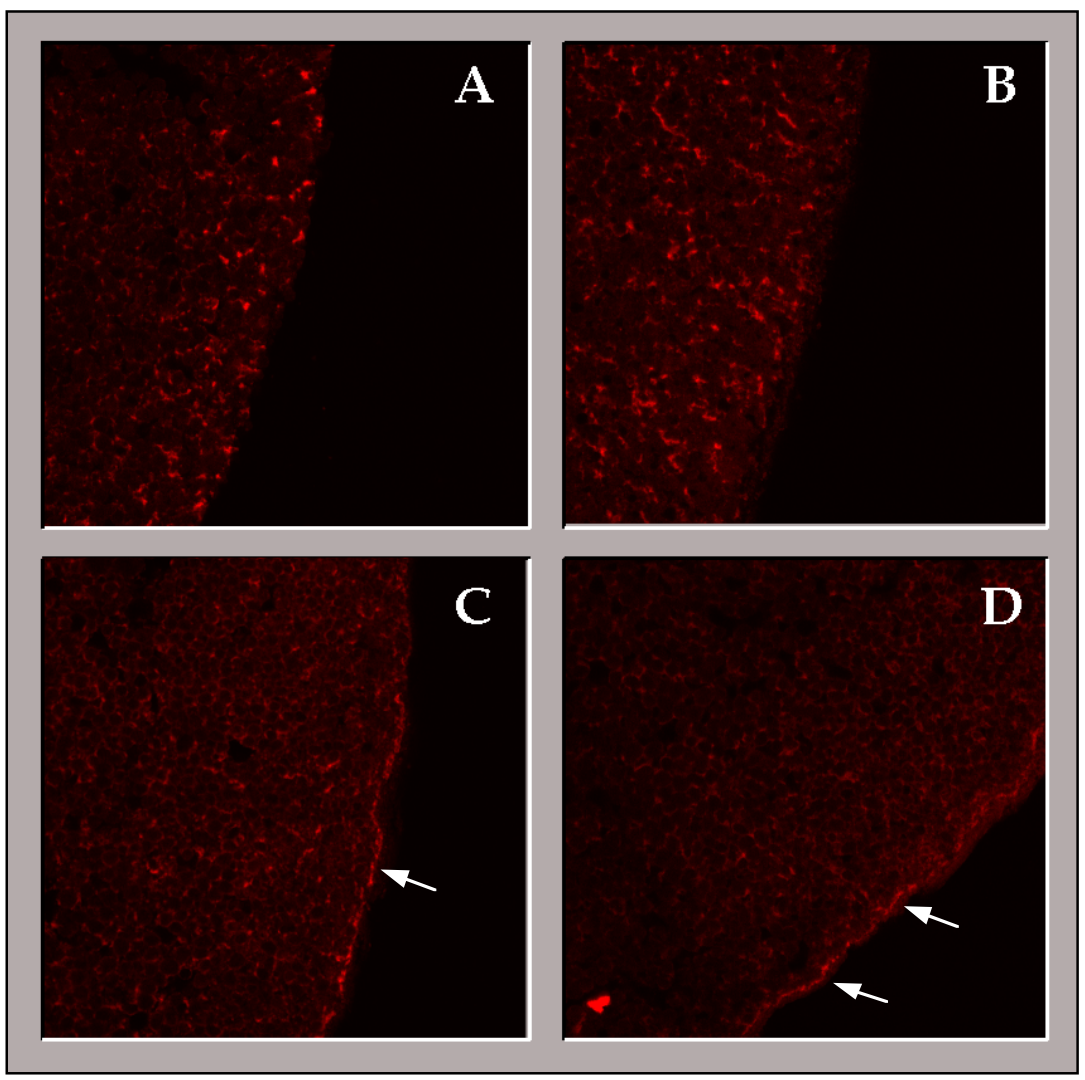

Figure 3.32: Immunolocalization of TM20 in oocytes. cRNA of TM20 was injected in oocytes and incubated for three days. Subsequently the oocytes were processed for immunocytochemistry. Control oocytes: water injected oocytes. A and B sections of oocytes injected with water. No specific label located in the plasmamembrane was observed. C and D oocytes injected with cRNA of TM20. A slight label of red fluorescence was observed in the plasmamembrane (arrows). 
detected by streptavidine conjugated with Rhodamine-Red.

The result of the immunohistochemistry showed the localization of TM20 in the plasma membrane of the oocytes (Fig. 3.32 C,D) as a thin red line on the border of the oocytes injected with cRNA of TM20. Although the labelling was of low intensity and not continuous, it was observed only in the oocytes injected with TM20 and not in the oocytes injected with water. The spotlike fluorescence inside the oocytes probably originates from crossreaction with the yolk proteins of the oocytes (Fig. 3.32 A,B). The overall result of the immunolocalization showed that TM20 seems to be inserted correctly in the plasma membrane of the oocyte.

\subsubsection{Oocytes injected with TM20 accumulate more radiolabeled IAA than control oocytes depending on time and pH}

The TM20 injected oocytes and the control oocytes were placed into $\mathrm{CHO}$ sample buffer and were incubated in the presence of ${ }^{3} \mathrm{H}$-IAA for $5 \mathrm{~min}, 15 \mathrm{~min}, 30 \mathrm{~min}$ and $60 \mathrm{~min}$. The oocytes were washed with sample buffer and the amount of ${ }^{3} \mathrm{H}$-IAA inside the oocytes was measured. Figure 3.33 shows the ${ }^{3} \mathrm{H}$-IAA accumulation in oocytes injected with TM20 and in control oocytes in relation to the incubation time.

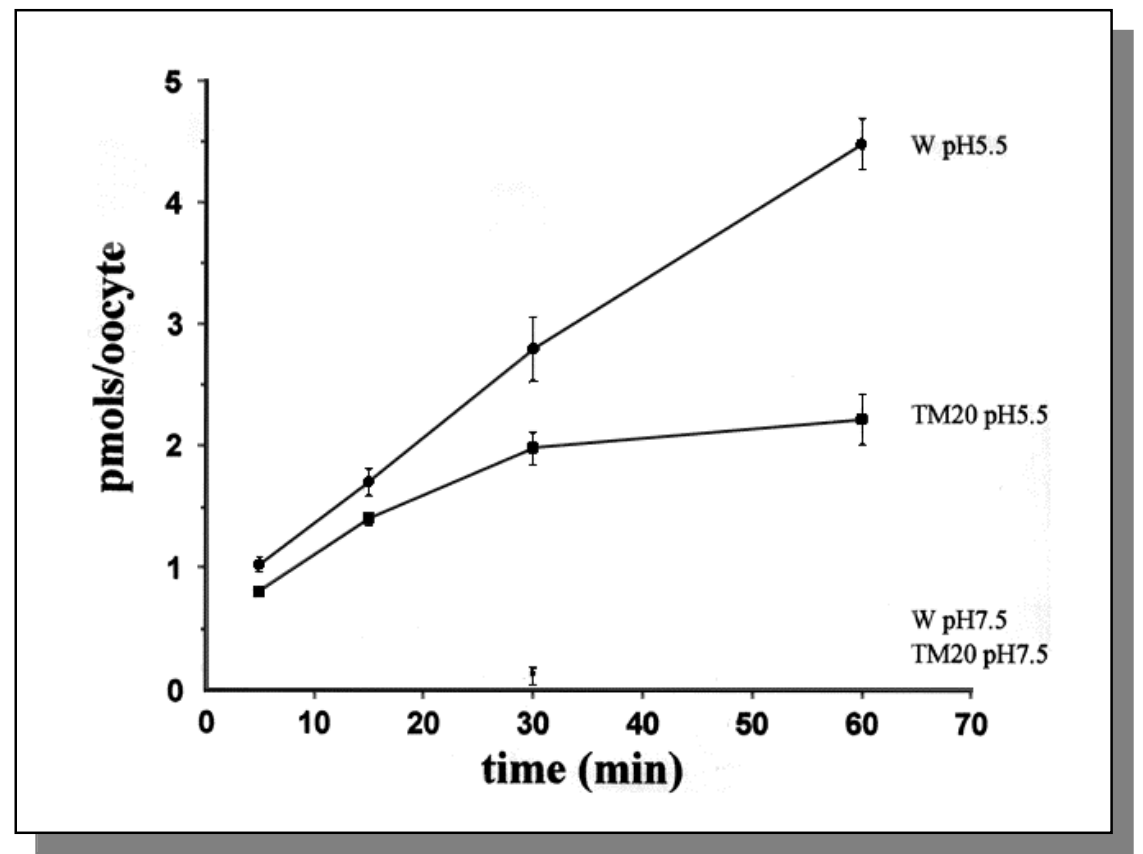

Figure 3.33: ${ }^{3} \mathrm{H}-\mathrm{IAA}$ accumulation in oocytes injected with TM20 and with water. The experiments shown in the graphic were carried out with two different $\mathrm{pH}$ (5.5 and 7.5). The accumulation of ${ }^{3} \mathrm{H}$-IAA in oocytes were measured after 5, 15, 30 and 60 min. The accumulations at $\mathrm{pH} 7,5$ were too low for a reliable measurment. At $\mathrm{pH} 5,5$ the oocytes injected with water had accumulated up to $50 \%$ more ${ }^{3} \mathrm{H}$-IAA than the oocytes injected with TM20. 
A difference in IAA accumulation between TM20 injected oocytes and control oocytes could be observed, which increases depending on the incubation time. This difference was $50 \%$ after $60 \mathrm{~min}$ of incubation. This difference in accumulation was only observed at $\mathrm{pH}$ 5.5. In contrast, incubation with a buffer at $\mathrm{pH} 7.5$, lead to a low uptake of total IAA in control oocytes as well as in TM20 injected oocytes and no reliable measurement was possible (Fig. 3.33). The same result was obtained incubating oocytes in sample buffer with $\mathrm{pH} 8.5$ (not shown). The data obtained by these results point to a possible transport activity of TM20 with IAA as substrate. However, it is not clear whether this accumulation difference is caused by a reduced uptake of IAA or efflux activity produced by TM20.

\subsubsection{IAA enters the oocytes by diffusion}

To determine the uptake of IAA into the oocytes, a diffusion study was carried out. For that purpose non-injected oocytes were incubated with different concentrations $(10 \mu \mathrm{M}, 50 \mu \mathrm{M}, 150 \mu \mathrm{M}$ and $500 \mu \mathrm{M})$ of ${ }^{3} \mathrm{H}$-IAA at pH5.5. The result of this experiment is shown in Figure 3.34. Even at high concentrations of IAA no saturation effect was observed and the uptake of IAA follows almost a linear increase. This result indicates that the uptake of IAA into the oocytes occurs by diffusion.

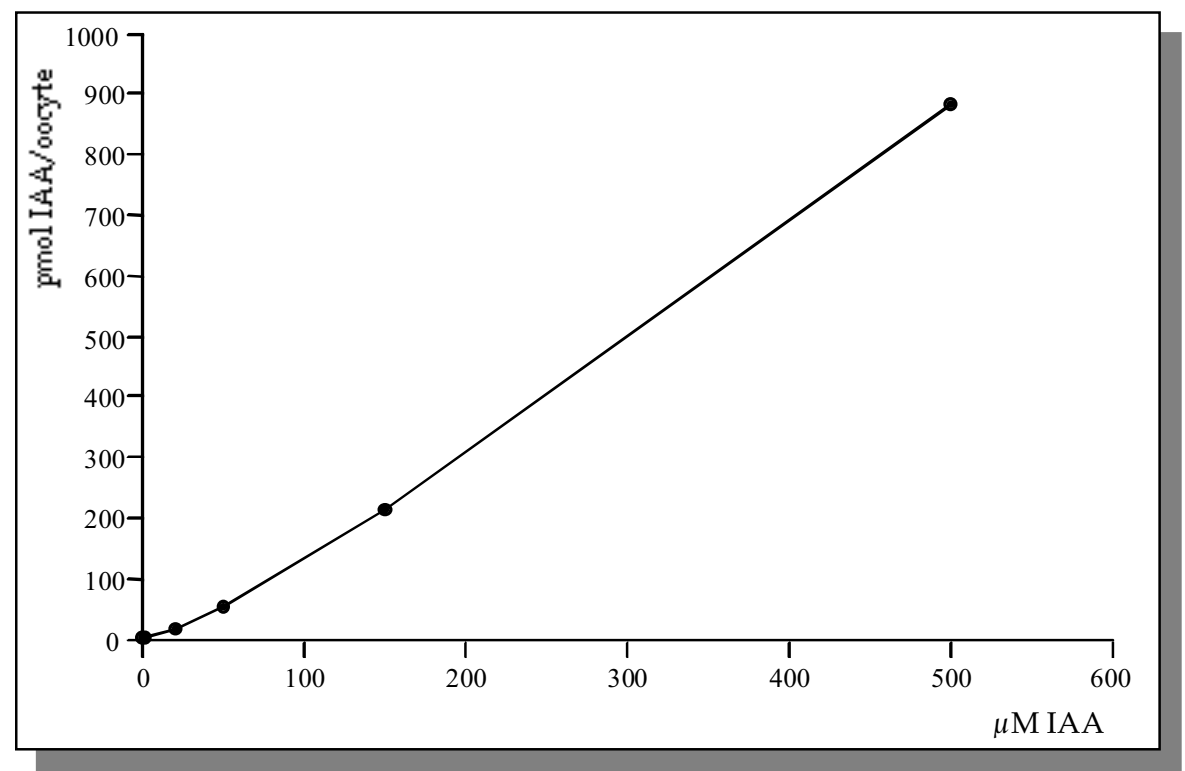

Figure 3.34: Diffusion driven uptake of ${ }^{3} \mathrm{H}$-IAA into oocytes. The accumulation of ${ }^{3} \mathrm{H}-\mathrm{IAA}$ is linear corresponding to the increasing IAA concentration. In the case of a transporter mediated uptake a saturation effect should be observed. 


\subsubsection{Addition of an auxin transport inhibitor (NPA) increases accumulation of 3H-IAA in TM20 injected oocytes}

In the case that the observed difference in IAA accumulation between control oocytes and oocytes injected with TM20 depends on auxin transport activity, this accumulation difference may be altered by addition of known auxin transport inhibitors.

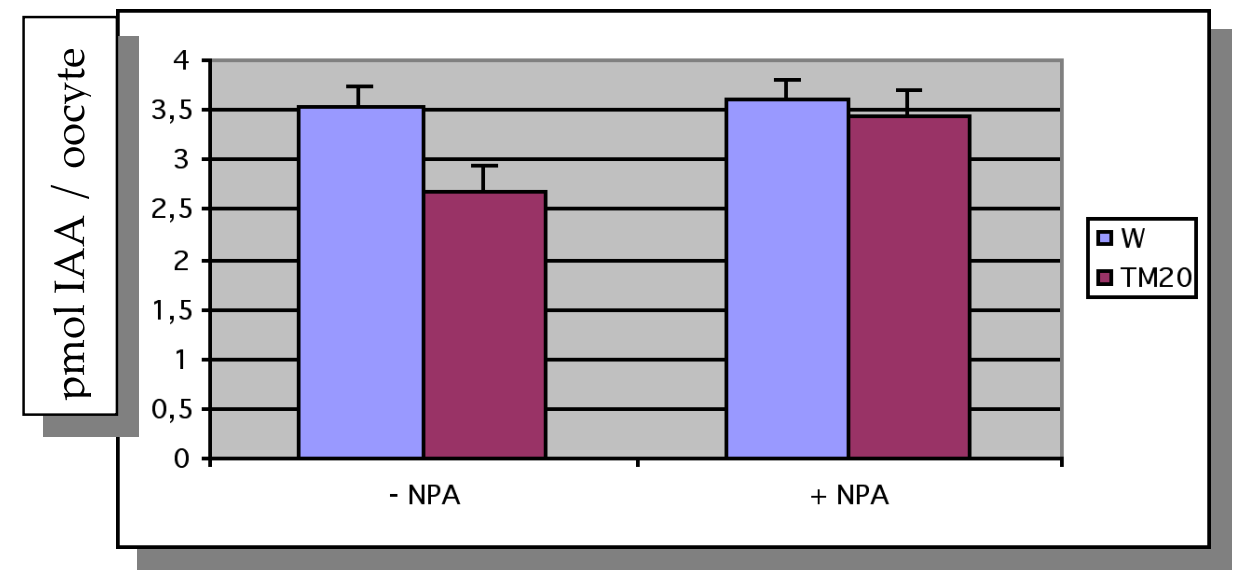

Figure 3.35: Change of IAA accumulation by addition of $5 \mu \mathrm{M}$ NPA. The transport assay was carried out with oocytes injected with water or with cRNA of TM20 in the presence of $5 \mu \mathrm{M}$ NPA and without NPA. The addition of NPA modifies the accumulation of IAA in oocytes injected with TM20.

N-1-Naphthylphtalamic acid (NPA) is known as a specific inhibitor of polar auxin transport. Even if its mechanism is not yet clear, a change in accumulation of IAA in TM20 injected oocytes could be evidence for the link between TM20 and auxin transport.

Figure 3.35 shows the accumulation of IAA in oocytes injected with TM20 (red bars) and in control oocytes (blue bars). The experiment was performed in the presence of $5 \mu \mathrm{M}$ NPA or without NPA addition. On the left, without NPA, there is a statistically significant difference in ${ }^{3} \mathrm{H}$-IAA accumulation after 1 hour of incubation in sample buffer, as observed before (in this case the difference was about $30 \%$ ). On the right the accumulation of ${ }^{3} \mathrm{H}$-IAA increases in the presence of NPA the accumulation of IAA in TM20 injected oocytes to almost the same levels as in control oocytes. 


\subsubsection{No difference in IAA accumulation is observed between control oocytes and oocytes injected with AtTM4}

The cRNA of the TM20 like gene AtTM4 from Arabidopsis thaliana (see 3.1.2) was injected in oocytes to determine whether a similar effect of different IAA accumulation could be detected as observed for TM20. Figure 3.36 shows the accumulation of ${ }^{3} \mathrm{H}$-IAA in control oocytes and oocytes injected with AtTM4. Although a slight difference in the total accumulation of ${ }^{3} \mathrm{H}$-IAA can be observed, the different accumulation was not dependent on incubation time and did not change at the different checkpoints. The accumulation of 3H-IAA in the control oocytes and the AtTM4 injected oocytes follows a linear increase according to a diffusion driven uptake (see.3.5.5). These results indicate that the observed difference between TM20 and AtTM4 affects the ability to transport IAA across the membrane of oocytes. Considering the described differences and the smaller size of the protein from Arabidopsis, additional cofactors may be necessary to cause a similar effect on the accumulation of IAA or a correct localization of AtTM4 in the plasma membrane of oocytes.

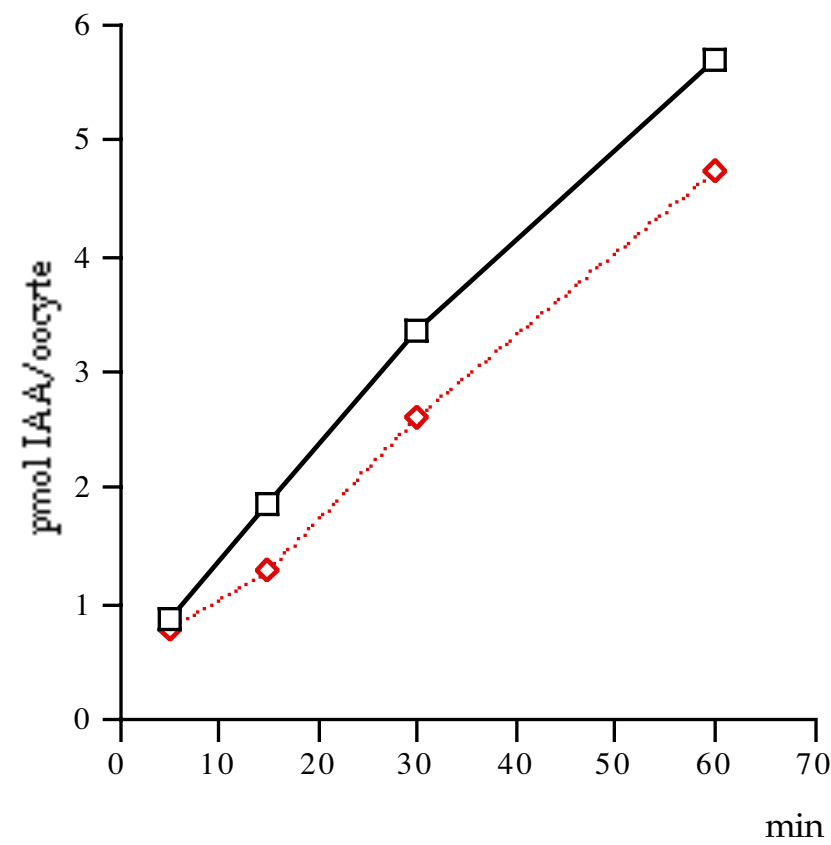

Figure 3.36: Accumulation of ${ }^{3} \mathrm{H}$-IAA in oocytes injected with AtTM4 (red curve) and water injected ooytes (black curve). No time dependent difference in ${ }^{3} \mathrm{H}-\mathrm{IAA}$ accumulation could be observed. 


\section{Discussion}




\section{Discussion}

In this work the function of the protein TM20 from maize, a new type of transmembrane protein, has been studied. Studies performed by Stiefel et al (1999) identified TM20, whose expression is inhibited in the dek mutant lachrima from maize. The ontogenesis of the lachrima mutant is blocked at the transition stage of embryo development and subsequently fails in the establishment of bilateral symmetry. Studies on wheat embryos cultured in the presence of auxin transport inhibitors or with an excess of auxin (Fischer and Neuhaus 1996; Hadfi et al. 1998) have demonstrated the link between embryo development and polar auxin transport. The embryos obtained in these studies displayed the same phenotype as the lachrima mutant. However, due to the limited number of maize embryo mutants and alleles of these mutants the genetic identity between TM20 and lachrima has not been confirmed completely. Although an Ac-element was detected in the promoter region of the TM20 gene and this is not expressed in the mutant, it can not be totally excluded that genes other than TM20 are also affected by any other transposon insertion and that these genes are responsible for the observed phenotype. Nevertheless, the clear inhibition of TM20 expression in the lachrima mutants and the transporter-like characteristics of TM20 suggest the involvement of the protein TM20 in the processes disturbed in lachrima. And based on the phenotype lachrima, it is supposed that one of these processes may be the polar transport of auxin.

\subsection{Presence of TM20 in other species}

Until now, TM20 had only been described in maize and no data on TM20 or similar proteins in other species were available in the databases. Due to the continuous up-dating and expansion of the databases, the homology searches performed during this work revealed genomic sequences from species such as Oryza sativa, Hordeum vulgare and Arabidopsis thaliana with open reading frames encoding putative proteins similar to TM20. The differences between TM20 and the deduced proteins are variable in the different species. The TM20 -like protein found in rice (OsTM20) has 20 hydrophobic regions predicted as transmembrane spanning 


\section{Discussion}

domains. Moreover, the arrangement of these transmembrane elements is exactly the same as in TM20 of maize. However, OsTM20 is much shorter than its counterpart in maize, which is basically due to the much reduced central loop between the eighth and ninth transmembrane element (see Fig. 3.3). The central loop of TM20 is thought to play an important role in the function of the protein since such a central hydrophilic region is characteristic for several membrane proteins with transporter function (Singer 1990; Marger and Saier 1993). So the reduced size of this central region in the rice homologue suggests a rather unimportant role of this protein structure in the protein function. In contrast, the maintenance of the arrangement of the 20 transmembrane elements in both proteins indicates the importance of the transmembrane organization for correct protein activity. In addition, the phosphorylation sites described for kinase C in TM20 are also conserved in OsTM20 and seem to play an important role in post-transcriptional processes or regulation of both proteins. Little is known about the putative homologue of TM20 in barley. But the high sequence homology of the fragment and the evolutionary proximity of barley to maize and rice indicate that TM20 is also found in barley.

Seven members of a gene family found in Arabidopsis encode putative proteins (Arab1-Arab6 and AtTM4), which are similar to TM20. These seven proteins have the same characteristics of several (4-7) ankyrin repeats followed by 4 hydrophilic regions at the C-terminal, which are predicted as transmembrane spanning domains. The N-terminal has low variability between the different proteins and only AtTM4 differs in the length of the $\mathrm{N}$-terminal from the consensus sequence by an additional 32 amino acids. Although different programmes for the prediction of signal peptide sequences were applied, no significant target sequence could be identified, but there is strong evidence for membrane localization of these proteins due to their four transmembrane spanning domains.

There is high homology between the amino acid sequences of Arab1-6 and AtTM4 in the region of the ankyrin repeats and between the transmembrane spanning elements. Multiple conserved motifs were identified, which form part of the ankyrin repeats. The differences between Arab1-Arab6 and AtTM4 are represented in a phylogenetic tree based on the alignment of the amino acid sequences of these proteins (Fig. 4.1). The differences between the proteins are based 
mainly on the regions with a higher variability. The first region comprises $60-70$ amino acids and is situated between the ankyrin repeats and the first transmembrane spanning element. This zone is highly hydrophilic and has an average positive charge. A second region where the amino acid sequences differ is the loop of 20 amino acids between the first and the second transmembrane segment. A high hydrophilicity characterizes this loop, which contains the conserved PPGG motif that is common to TM20, OsTM20 and the proteins found in Arabidopsis, except Arab3 and Arab6.

Since the estimated size of TM20 differs from the size of the proteins of Arabidopsis, and the homology of the amino acid sequences is restricted to a small region, the most common feature between TM20 and these proteins is the organization of the transmembrane elements. The arrangement of the four transmembrane domains in Arab1-Arab6 and AtTM4 is the same as in the five repeated groups of the transmembrane domains of TM20. Moreover, the region of highest homology between TM20 and the proteins from Arabidopsis is located in this area, between the first and the second transmembrane element. The structure of four transmembrane spanning elements have been described for a family of proteins, the connexins, that allow the formation of gap-junctions in animal cells (Kumar and Gilula 1996). Up to now, no connexin protein was found in plants. However, one important feature of the connexins is a number of cystine residues, which are not present in TM20 or Arab1-6 and AtTM4. The presence of ankyrin repeats at the Nterminal, described as sites of protein-protein interaction (Batchelor et al. 1998; Hartshorne 1998) indicates that the proteins from Arabidopsis may be able to interact with other proteins or between themselves. These interactions may lead to the formation of protein complexes displaying structures with a similar number of transmembrane spanning domains observed for TM20 from rice and maize. Alternatively, the five repeats of transmembrane domains of TM20 may be cleaved after transcription and result in smaller proteins of a size corresponding to those of Arabidopsis encoded by single genes. The fact that the protein detected in western blot analysis had a molecular weight of about $50 \mathrm{KDa}$ supports this hypothesis.

The genomic organization in tandem in the case of Arab2:Arab5 and Arab3:Arab6 suggest that during evolution these genes may have changed from 
encoding one large protein to encoding smaller subunits of a protein, which improves the function by forming complexes of these subunits. The phylogenetic tree in Figure 4.1A is based on the alignment of the single modules of four transmembrane segments of TM20 and OsTM20 with Arab1-6 and AtTM4. While the modules of TM20 and OsTM20 show high similarity between each other, in Arabidopsis the proteins seem to be phylogenetically more distant. In this context, the proteins encoded by genes in tandem (Arab3:Arab6 and Arab2:Arab5) are closer phylogenetically (Fig. 4.1A). The same relations are found comparing the genomic sequences of Arab1-6 and AtTM4 (Fig. 4.1B).

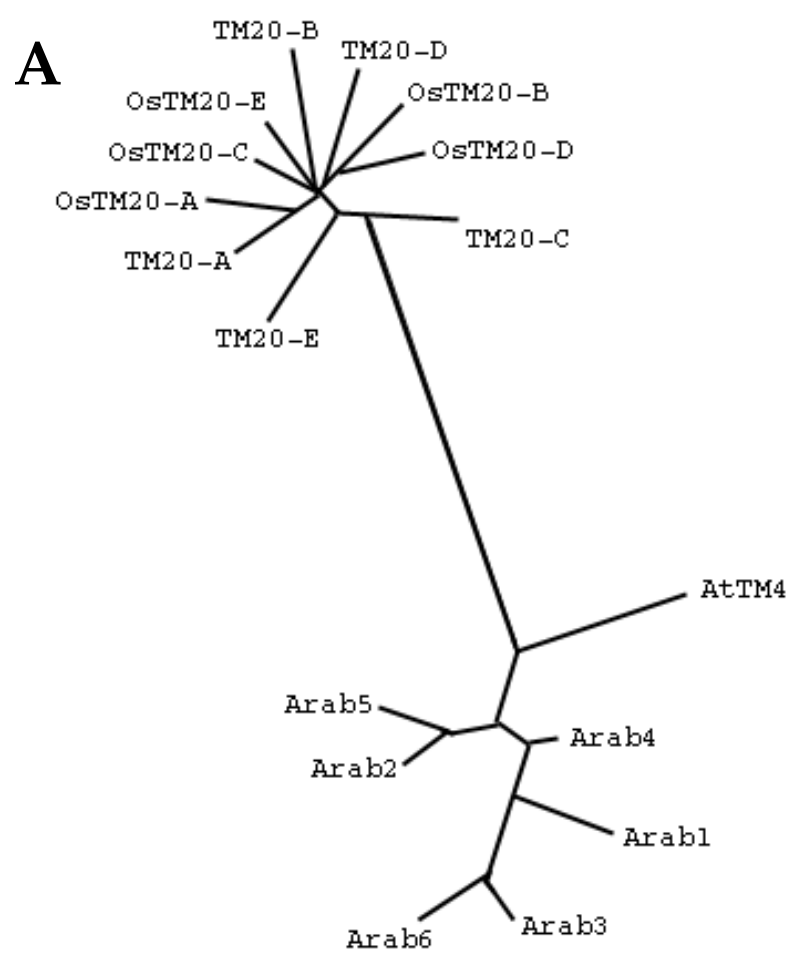

B

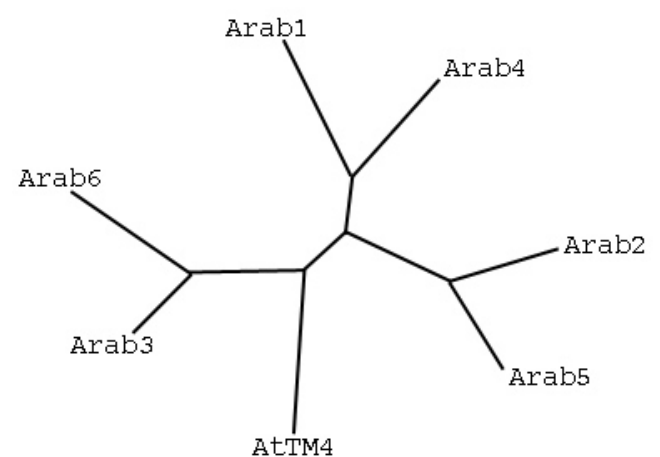

Figure 4.1: Alignment tree of A: proteins from Arabidopsis Arab1-6 and AtTM4 and the different modules of 4 transmembrane domains from TM20 and OsTM20 and B: the genomic sequences of Arab1-Arab6 and AtTM4.

Nevertheless, the difference between TM20 from monocots and TM20 from dicotyledons is considerable (Fig. 4.1A), but not surprisingly, since a variety of genes have been described which differ within different plant classes and species based on their phylogenetic distance (Stranathan et al. 1989; Vinogradov 2001). The fact that the first module of TM20, TM20-A, is more similar to OsTM20-A than to TM20-B-E may indicate that the multiplication of the modules occurred after the evolutionary separation of monocotyledons and dicotyledons (Fig. 1.4A). 


\subsection{Localization of TM20 in the maize embryo}

During plant development, tissue restricted gene expression plays an important role in the co-ordination of developmental processes. Moreover, the function of a protein is, in general, tightly correlated with its localization in tissues or at the subcellular level, which is once more linked to its physicochemical nature. Consequently, an important part of this work was dedicated to determining the localization of the protein TM20 in maize embryo tissue and at the subcellular level.

Stiefel et al (1999) showed with northern blot analysis and in situ hybridizations on embryo sections that the TM20 gene is expressed a few hours after fertilization. The transcript of TM20 could be detected up to 20 days after pollination and is accumulated in the leaf primordia, the root and also in meristematic and provascular tissue. To detect the protein TM20, affinity purified polyclonal antibodies were used in in situ hybridization of embryo sections and in whole mount hybridization. Previously the antibodies were used in western blot and dot blot analysis. The dot blot assays showed the association of TM20 to membranes since the affinity purified polyclonal antibodies specific for TM20 detected the protein mainly in the microsomal fractions of maize embryo protein extractions. Furthermore, these assays revealed a decrease of antibody affinity when the protein samples were treated with denaturating agents such as SDS. The fact that the antibody recognition of denaturated TM20 was reduced, it complicated the analysis by western blot. The protein detected in the western blot is much smaller than that estimated from the deduced amino acid sequence. A reduced protein size can be due to protein degradation during extraction or the cross reaction of the antibodies with epitopes that do not belong to TM20. However, post-transcriptional modification including the proteolytic cleavage of TM20 also explains the reduced size of the protein detected in western blot analysis. In this case, the western blot results support the hypothesis mentioned before, that TM20 may be modified and lead to a protein size similar to the proteins of Arabidopsis Arab1-Arab6 and AtTM4.

TM20 was detected in cultured BMS cells and embryos from maize. The localization of TM20 observed in maize embryos at different developmental stages (10DAP, 15DAP and 20DAP) is in accordance with the accumulation of TM20 


\section{Discussion}

transcripts. At 10DAP TM20 is located basically in the apical part of the embryo axis, including the developing coleoptile and the shoot apical meristem, and also in the radicle. Later, at 15DAP, TM20 is also observed in the shoot apical meristem, the coleoptile and the radicle. About 20DAP TM20 is localized mainly in the young leaves and the shoot apical meristem, but it is also present in cell layers surrounding the vascular tissue along the embryo apical-basal axis. In the radicle, TM20 was observed in vascular and provascular cells and in cortical cells.

It has been shown that the vascular tissue is the main route of apical-basal polar auxin transport in plants and even if the molecular details remain hypothetical, the apical-basal transport of IAA itself is experimentally well established (Jones 1990; Berleth et al. 2000). However, little is known about auxin transport during embryogenesis, due to the reduced size of the embryos and the difficulties of applying the common assays of auxin transport measurement. On the other hand the role of auxin in the formation of vascular strands and vein patterning in plants is widely documented (Mattsson et al. 1999; Sieburth 1999; Berleth and Mattsson 2000). The differentiation of vascular tissue is directly linked to polar auxin transport and the IAA flow through differentiating vascular tissue has been confirmed experimentally (Gersani and Sachs 1984). Moreover, several Arabidopsis mutants that are related to defective auxin transport or perception, as bdl1, pin1, emb30/gn, mp and lop1, also have alterations in vascularization (Berleth, Mattsson et al. 2000). The localization of TM20 in the tissues of the vascular system, therefore, is consistent with the site of the main flux of polar auxin transport.

The fact that auxin is transported from the sites of biosynthesis in young leaves and meristematic tissues through the whole plant to the root tip is well known and has been studied extensively (Lomax et al. 1995). Although genes coding for putative components of the cell-to-cell transport system have been cloned only in the last years (Bennett et al. 1996; Gälweiler et al. 1998; Luschnig et al. 1998; Marchant et al. 1999), the hypothesis that the polarity of transport is based on the localization of transporter proteins at the apical and basal part of the cells, had been proposed long before (Rubery and Sheldrake 1974; Raven 1975; Lomax, Muday et al. 1995). Three putative auxin carrier proteins have been cloned from Arabidopsis. The AUX1 gene codes for a presumptive auxin influx carrier, while AtPIN1 and AtPIN2 (EIR1, AGR1, 
WAV6-52) have been suggested to act as auxin efflux carriers. In indirect immunolabeling studies it has been observed that AUX1 is localized in the apical plasma membrane of the root protophloem and the cells of the lateral root cap (Swarup et al. 2000; Swarup et al. 2001). AtPIN2 is localized at the plasma membrane in the upper part of the cells of the root cortex, whereas AtPIN1 has been observed in the plasma membrane of the basal part of parenchymatous xylem cells (Gälweiler, Guan et al. 1998; Müller et al. 1998). No protein with similar characteristics has previously been described in maize or other plant species. The subcellular localization of TM20 in cells of maize embryos, reported here, is similar to the localization observed for AtPIN2 and AUX1 in Arabidopsis roots. TM20 was observed to be distributed asymmetrically in the upper part of the cells, causing a polarization effect in the cell. In contrast to AtPIN2 and AUX1, TM20 localization is not restricted exclusively to the apical plasma membrane, but it was also observed below the plasma membrane in a more diffused manner indicating that TM20 may also be found in vesicles directed to the plasma membrane. This points to a dynamic regulation of TM20 and suggests a high trafficking of TM20 to the plasma membrane.

Surprisingly, the subcellular distribution observed for TM20 is dependent on the developmental stage of the maize embryo and the differential state of the cells. In cells of the coleoptilar and the shoot apical meristem of 10DAP embryos, TM20 is distributed over the whole cell lumen, except in the nucleus. The same distribution is found in undifferentiated meristematic cells of more developed embryos (20DAP). The asymmetric distribution observed in more differentiated tissues seems to take place parallel to the formation of the apical basal asymmetry of the embryo and cell differentiation. Even though little is known about the auxin levels in embryos at the transition and coleoptilar stage, polar auxin transport is an essential process for the formation of apical-basal asymmetry (Liu et al. 1993) and has to be established before or during this developmental stage. The definition of a polar localization of TM20 dependent on the developmental stage may overlap with the formation of the auxin gradient and relate the localization of TM20 to this process. A similar pattern was also found in 10DAP maize embryos, using specific antibodies against a plasma membrane located ATPase (Villalba et al. 1991). In the more differentiated scutellum cells the ATPase is located in the plasma membrane, while ATPase distribution in 


\section{Discussion}

cells of the embryo axis is equal to the localization of TM20 in the same cell type. These results indicate that some membrane integral proteins are positioned in the plasma membrane depending on the state of differentiation of the cells.

\subsection{The capacity of TM20 to transport IAA across membranes}

The expression of membrane transport genes in heterologous systems such as oocytes of Xenopus laevis, yeast, COS (cells from green monkey kidney) and insect cells is an important approach to study the functional properties of the corresponding transporter proteins. Here we report the expression of cRNA of TM20 in oocytes of Xenopus laevis to test for the ability of TM20 to transport IAA through membranes. The results obtained from these studies showed that radiolabeled IAA enters the oocytes dependent on the $\mathrm{pH}$ of the sample buffer. At $\mathrm{pH} 5.5$ in the outer media, IAA is mainly protonated and enters the oocyte by diffusion. Inside the oocyte, at higher $\mathrm{pH}$ the anionic form is favoured and the charged IAA molecule is trapped and accumulates in the cell (Lomax, Muday et al. 1995; Delbarre et al. 1996). The lack of accumulation using sample buffers with $\mathrm{pH} 7.0$ and $\mathrm{pH} 8.5$ is consistent with this model. Comparing the accumulation of IAA in oocytes injected with TM20 and control oocytes injected with $\mathrm{H}_{2} \mathrm{O}$, it was observed that the control oocytes accumulated up to 50\% more radiolabeled IAA than the oocytes injected with TM20. These results together with the diffusion studies indicate a transport activity of TM20 injected oocytes that could not be observed in the control oocytes. A possible interpretation of this result is given in a schematic model (Fig. 4.2). IAA enters equally by diffusion in both types of oocytes (Fig. 4.2A), TM20 injected and $\mathrm{H}_{2} \mathrm{O}$ injected, and is accumulated inside the cell (Fig. 4.2B). The presence and the activity of TM20 in the plasma membrane facilitate the efflux of IAA out of the oocyte (Fig. 4.2C), which works against the entering IAA molecules. This model is consistent with the increase of the difference in IAA accumulation dependent on the incubation time and duration of transport (60min) (Fig. 3.31).

The addition of the known auxin transport inhibitor NPA leads, under the same experimental conditions, to an adjustment of the IAA accumulation in TM20 injected oocytes and in control oocytes to equal levels. 
The identification and characterisation of transport systems in plants is complex and the results obtained by the expression in heterologous systems have to be interpreted carefully (Barbier-Brygoo et al. 2001). For instance a $\mathrm{K}^{+}$-channel from stomatal guard cells showed pronounced differences in activity between the native channel and the subcloned $\alpha$-subunits expressed in Xenopus oocytes (Dreyer 1999). Several factors may interfere in the correct development of transport activity in heterologous systems, e.g. the incorrect maturation of the protein or the misguided targeting to the plasma membrane. In some cases, the correct transport activity requires interaction with plant specific proteins or a cell-type-specific posttranslational modification. The IAA transport effect observed in oocytes expressing TM20 indicates that no additional plant specific co-factor is necessary for the transport activity and that the TM20 expressing oocytes provide the machinery for the transport of IAA across the membrane. Nevertheless, Xenopus oocytes are an animal system and a distinct activity of TM20 in plant cells can not be excluded by the data presented here.

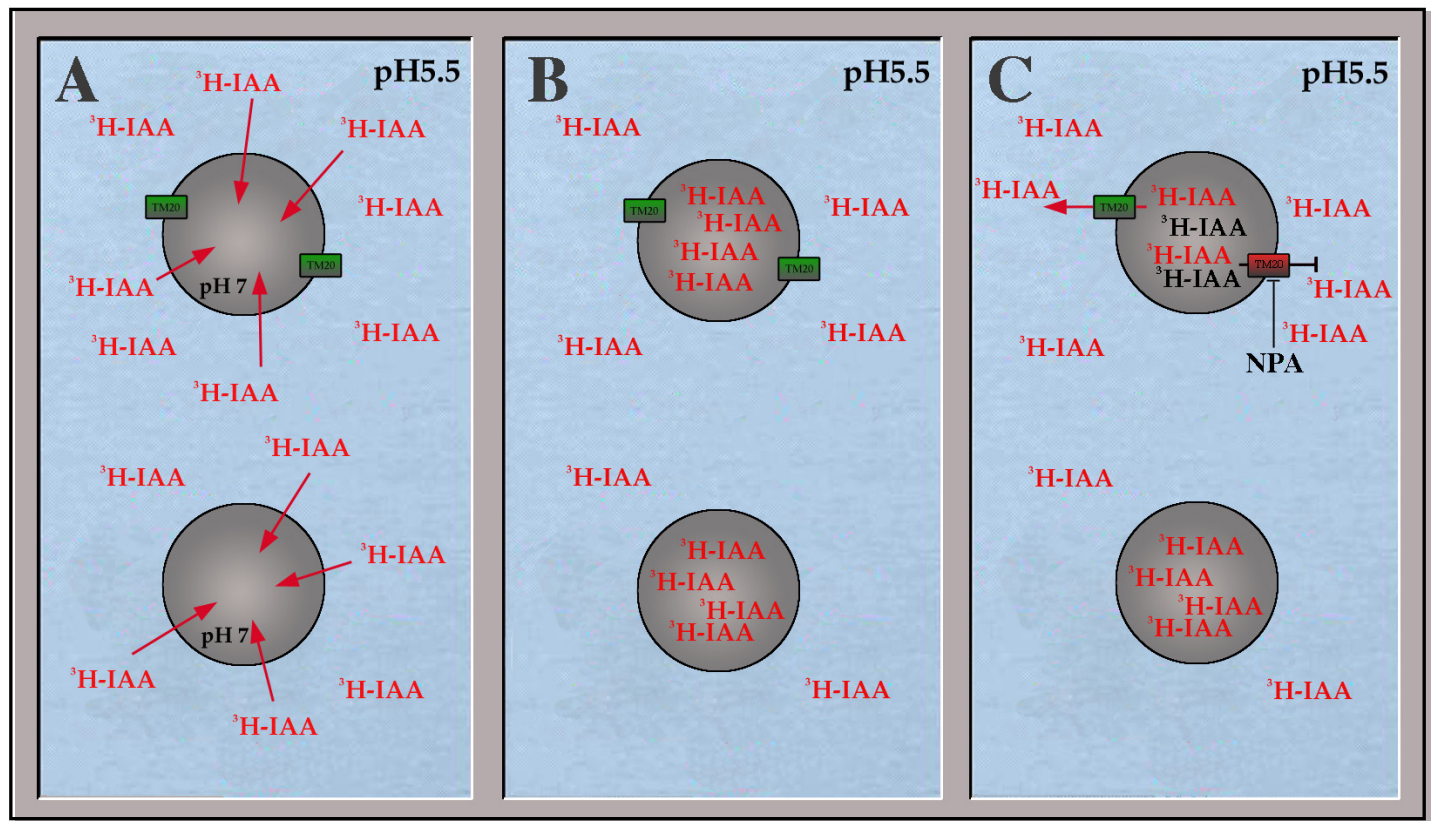

Figure 4.2: Model of the process of different ${ }^{3} \mathrm{H}$-IAA accumulation in oocytes injected with TM20 and control oocytes. A: ${ }^{3} \mathrm{H}-\mathrm{IAA}$ enters by diffusion the oocytes and B: accumulates inside. C: In the control oocytes (below) the IAA remains retained in the cell, while the oocytes injected with TM20 (above) gained the ability to expulse IAA. The addition of NPA blocks the TM20 mediated IAA transport (red box) and IAA accumulates in TM20 injected oocytes in similar amounts such as in the control oocytes (IAA in black). 


\section{Discussion}

The transport specifity of TM20 for the substrate IAA is also not totally clear. Even if the observed effect of the specific auxin transport inhibitor NPA on the IAA accumulation points to an IAA transport, the molecular processes of transport inhibition by NPA are not resolved yet. Recent studies indicate that chemical inhibitors that were originally described as specific auxin transport inhibitors e.g. TIBA, interfere in membrane targeted vesicle trafficking (Geldner et al. 2001). Accordingly, the described effect of NPA on IAA accumulation may be based on the failure of correct membrane trafficking. Despite this, the inhibitory effect of NPA indicates that the observed transport effect is related to the presence of TM20 in the membrane of the oocyte.

It can not be excluded that TM20 is a transporter with a distinct substrate specifity other than IAA. Various molecules such as the amino acid tryptophan and indole, precursors in the biosynthesis of IAA, are similar to IAA and may be the transported compound. Several proteins that transport tryptophan specifically in yeast, human and rat have been described (TAT1; (Kim do et al. 2002), as well as amino acid transporters with a low selectivity towards the amino acid side chains, termed AAPs (amino acid permease). The AtAAP1 protein is found exclusively in seeds, and it is suggested that it plays an important role in the supply of developing embryos (Frommer et al. 1993; Hirner et al. 1998), whereas AtAAP2 is located in the vascular tissue (Frommer et al. 1995). However, beside the general hydrophobic character and the high number of putative transmembrane domains of these proteins, no significant homology between TM20 and these proteins could be found. Moreover, considering the important role of auxin in the regulation of growth and development of the plant, it is more likely that auxin carriers are characterized by a high substrate specifity.

TM20 possesses a motif described as signature1 of a family of sodium:solute symporters (Prosite: PDOC00429; PS00456) (Reizer et al. 1994). These symporters mediate the transport of a variety of molecules through the membrane, coupled with the uptake of sodium ions. A variety of these symporters have been found in mammals and prokaryotes and are predicted to contain at least ten membrane spanning domains. Although in TM20 only one of the two signatures that characterize this symporter family was found, this similarity together with the 
multiple transmembrane spanning domains support the carrier character of the TM20 structure.

The difficulties of the functional study of plant-specific transport systems are reflected in the lack of data on functional characterisation of the putative auxin carriers AtPIN1, AUX1 and AtPIN2 (the latter one is identical to EIR1, AGR1 and WAV6). Only the groups that identified EIR1 and AGR1 give experimental data of physiological studies (Chen et al. 1998; Luschnig, Gaxiola et al. 1998; Utsuno et al. 1998). Yeast strains expressing EIR1 have been shown to be more resistant against toxic 5-fluoro-indole and 5-DL-fluoro-tryptophan (Luschnig, Gaxiola et al. 1998). Whether these results are due to the prevention of the uptake or to the facilitated efflux of the toxic compounds is not clear. In the case of AGR1 the authors observed that agr1 mutants retain more preloaded ${ }^{3} \mathrm{H}$-IAA in their root tips than in the wild type. After 2 hours of washing the preloaded root tips of agr1 mutants retained most of the ${ }^{3} \mathrm{H}$-IAA, while wild type roots only retained up to $60 \%$ (Chen, Hilson et al. 1998). Many unknown processes in the cell could be the origin of these results; different approaches to resolve the questions of substrate specifity, regulation etc, are necessary to confirm the results.

\subsection{Is TM20 part of the auxin transport system?}

Several indications point to the involvement of TM20 in polar auxin transport. These indications are based on the phenotype of lachrima mutants that is similar to mutants with disturbed auxin signalling, the localization of TM20 in tissue of supposed high auxin flux and the ability of oocytes expressing TM20 to transport IAA across the membrane.

As mentioned before, the phenotype of lachrima mutants is arrested in the transition stage and they do not develop bilateral symmetry. The same occurs to embryos cultured in the presence of auxin transport inhibitors or growing with an excess of exogenous auxin (Liu, Xu et al. 1993; Fischer and Neuhaus 1996). The arrest at early stages of embryogenesis and the failure in the transition to a bilateral symmetrical structure has also been described for Arabidopsis mutants defective in the $A B P 1$ gene that binds auxin and it is suggested that it mediates auxin induced 


\section{Discussion}

cell processes (Chen et al. 2001). The phenotypes produced by perturbations involving auxin may interfere at different levels such as auxin synthesis, polar auxin transport, auxin perception and auxin metabolism (Berleth and Sachs 2001). This is the case for mutants mentioned previously, bodenlos, monopteros, pin1-1 and emb30/gnom, which are affected at distinct levels of auxin action. These observations suggest that the characteristics displayed by the phenotype of lachrima are due to a disturbance in auxin action.

The localization of TM20 in the vascular tissue, leaf primordia and shoot meristem places TM20 in tissues and organs that are known as sites of elevated auxin transport or biosynthesis respectively (Mathysse and Scott 1984; Jones 1990; Ueda et al. 1991). At the subcellular level TM20 was located at the apical end of the cells, which is the same location observed for AtPIN2 in the Arabidopsis root (Müller, Guan et al. 1998). AtPIN2 may mediate basipetal auxin transport from the root tip to the shoot through the root parenchyma. Recent studies on AUX1 suggest the existence of two distinct transport pathways in the Arabidopsis root apex (Swarup, Friml et al. 2001). This result may indicate the existence of alternative transport routes through distinct tissues and mediated by distinct carrier proteins beside the known main streams of auxin in the plant. Accordingly, AtPIN1 and AtPIN2 are proposed to transport auxin in two different directions in the plant through different cell types (Gälweiler, Guan et al. 1998; Müller, Guan et al. 1998). In this context, a third protein of the PIN family has been described recently (Friml et al. 2002). AtPIN3 seems to mediate lateral auxin transport regulating tropism in the Arabidopsis root and change the location depending on the direction of tropism. TM20 has no similarity to the putative auxin carriers of Arabidopsis, and may be part of an auxin transport system that operates in an alternative transport pathway not described up to now. Since no direct auxin transport activity was shown for the PIN proteins, it is also possible that TM20 acts in the same transport way interacting with the PIN proteins.

In addition, alternative auxin transport systems may operate not only in distinct cell types, but also at different stages of development. The LOP1 gene has been shown to be involved in auxin transport. The phenotype of the lop 1 mutant displays abnormal shoot, root and vascular patterning but its cotyledon phenotype is normal. The authors suggest that LOP1 functions primarily in the post-embryonic 
phase of development (Carland and McHale 1996). Moreover, the study of the pathways of IAA biosynthesis in the plant has been shown to be diverse and to depend on the developmental stage. During early embryogenesis, a Trp-dependent pathway is favoured over a Trp-independent synthesis, providing sufficient free IAA required for the developmental processes at this stage (Normanly and Bartel 1999). If we consider the main expression of TM20 in the very early stages of embryogenesis shown by northern blot analysis (Stiefel et al. 1999), TM20 may form part of an auxin transport system active during embryogenesis and adapted to the necessities of the developing embryo, and different to the transport system in the adult plant.

In conclusion, during early embryogenesis of monocotyledons, TM20 seems to catalyze auxin-export in the reaction system of polar auxin transport. 



\section{Literature}




\section{Literature}

Abel, S. and A. Theologis (1996). "Early genes and auxin action." Plant Physiol 111(1): 9-17.

Ballas, N., L.-M. Wong, K. Malcom and A. Theologis (1995). "Two auxin-responsive domains interact positively to induce expression of the early indoleacetic acidinducible gene PS-IAA4/5." Proc Natl Acad Sci U S A 86: 3483-3487.

Ballas, N., L.-M. Wong and A. Theologis (1993). "Identification of the auxinresponsive element, $A u x R E$, in the primary indoleacetic acid-inducible gene PS-IAA4/5, of Pea (Pisum sativum)." J Mol Biol 233: 580-596.

Barbier-Brygoo, H., F. Gaymard, N. Rolland and J. Joyard (2001). "Strategies to identify transport systems in plants." Trends in Plant Science 6(12): 577-585.

Batchelor, A. H., D. E. Piper, F. C. de la Brousse, S. L. McKnight and C. Wolberger (1998). "The structure of GABPalpha/beta: an ETS domain- ankyrin repeat heterodimer bound to DNA." Science 279(5353): 1037-41.

Bennett, M. J., A. Marchant, H. G. Green, S. T. May, S. P. Ward, P. A. Millner, A. R. Walker, B. Schulz and K. A. Feldmann (1996). "Arabidopsis AUX1 gene: a permease-like regulator of root gravitropism." Science 273(5277): 948-50.

Berleth, T. and G. Jürgens (1993). "The role of the monopteros gene in organizing the basal body region of the Arabidopsis embryo." Development 118: 575-587.

Berleth, T. and J. Mattsson (2000). "Vascular development: tracing signals along veins [In Process Citation]." Curr Opin Plant Biol 3(5): 406-11.

Berleth, T., J. Mattsson and C. S. Hardtke (2000). "Vascular continuity and auxin signals." Trends in Plant Science 5(9): 387-393.

Berleth, T. and T. Sachs (2001). "Plant morphogenesis: long-distance coordination and local patterning." Curr Opin Plant Biol 4(1): 57-62.

Bertran, J., S. Magagnin, A. Werner, D. Markovich, J. Biber, X. Testar, A. Zorzano, L. C. Kuhn, M. Palacin and H. Murer (1992a). "Stimulation of system y(+)-like amino acid transport by the heavy chain of human $4 \mathrm{~F} 2$ surface antigen in Xenopus laevis oocytes." Proc Natl Acad Sci U S A 89(12): 5606-10.

Bertran, J., A. Werner, M. L. Moore, G. Stange, D. Markovich, J. Biber, X. Testar, A. Zorzano, M. Palacin and H. Murer (1992b). "Expression cloning of a cDNA from rabbit kidney cortex that induces a single transport system for cystine and dibasic and neutral amino acids." Proc Natl Acad Sci U S A 89(12): 5601-5. 


\section{Literature}

Birnboim, H. C. and J. Dolly (1979). "A rapid alkaline extraction procedure for screening recombinant plasmid DNA." Nucleic Acids Res 7: 1513-1523.

Bommert, P. and W. Werr (2001). "The expression pattern of LIPID TRANSFER PROTEIN2 (LTP2) gene indicates regionalization in the proembryo and confines the coleoptile to be in lineage with the scutellum." Maize News Letters 75.

Bommert, P. and W. Werr (2001). "Gene expression patterns in the maize caryopsis: clues to decisions in embryo and endosperm development." Gene 271(2): 13142.

Carland, F. M. and N. A. McHale (1996). "LOP1: a gene involved in auxin transport and vascular patterning in Arabidopsis." Development 122(6): 1811-9.

Chandlee, J. M. (1991). "Analysis of developmentally interesting genes cloned from higher plants by insertional mutagenesis." Dev. Genet. 12: 261-271.

Chen, J.-G., H. Ullah, J. C. Young, M. R. Sussmann and A. M. Jones (2001). "ABP1 is required for organized cell elongation and division in Arabidopsis embryogenesis." Genes and Development 15: 902-911.

Chen, R., P. Hilson, J. Sedbrook, E. Rosen, T. Caspar and P. H. Masson (1998). "The arabidopsis thaliana AGRAVITROPIC 1 gene encodes a component of the polar-auxin-transport efflux carrier." Proc Natl Acad Sci U S A 95(25): 15112-7.

Clark, J. K. (1996). Maize embryogenesis mutants. Embryogenesis: The generation of a plant. Oxford, Scientific Publishers: 89-112.

Clark, J. K. and W. F. Sheridan (1991). "Isolation and characterisation of 51 embryospecific mutations of maize." The Plant Cell 3: 935-951.

Davies, P. (1995). Plant Hormones. Dordrecht, The Netherlands, Kluwer Academic Publishers.

Delbarre, A., P. Muller and J. Guern (1998). "Short-Lived and Phosphorylated Proteins Contribute to Carrier-Mediated Efflux, but Not to Influx, of Auxin in Suspension-Cultured Tobacco Cells." Plant Physiol 116(2): 833-44.

Delbarre, A., P. Müller, V. Imhoff and J. Guern (1996). Planta 19: 532.

Doerner, P. (2000). "Does auxin provide positional cues ?" Curr Biol 10: R201-R203.

Dolan, L. (1998). "Pointing roots in the right direction: the role of auxin transport in response to gravity." Genes Dev 12(14): 2091-5.

Dolan, L., K. Janmaat, V. Willemsen, P. Linstead, S. Poethig, K. Roberts and B. Scheres (1993). "Cellular organisation of the Arabidopsis thaliana root." Development 119(1): 71-84. 
Dreyer, I. (1999). "Identification and characterization of plant transporters using heterologous expression systems." J. Exp. Bot. 50: 1073-1087.

Esau, K. (1960). Anatomy of Seed Plants. New York, John Wiley \& Sons.

Estelle, M. (1996). "Plant tropisms: the ins and outs of auxin." Curr Biol 6(12): 1589-91.

Estelle, M. (1998). "Polar auxin transport. New support for an old model [In Process Citation]." Plant Cell 10(11): 1775-8.

Estelle, M. (2001). "Plant hormones Transporters on the move." Nature 413(6854): 374-5.

Feldmann, K. A. (1991). "T-DNA insertion mutagenesis in Arabidopsis: Mutational spectrum." Plant Journal 1: 71-82.

Fischer, C. and G. Neuhaus (1996). "Influence of auxin on the establishment of bilateral symmetry in monocots." Plant J. 9: 659-669.

Franzmann, L. H., E. S. Yoon and D. W. Meinke (1995). "Saturating the genetic map of Arabidopsis thaliana with embryonic mutations." The Plant Journal 7: 341.

Friml, J., J. Wisnieswka, E. Benková, K. Mendgen and K. Palme (2002). "Lateral relocation of auxin efflux regulator PIN3 mediates tropism in Arabidopsis." Nature 415: 806-809.

Frommer, W. B., S. Hummel and J. W. Riesmeier (1993). "Expression cloning in yeast of a cDNA encoding a broad specificity amino acid permease from Arabidopsis thaliana." Proc Natl Acad Sci U S A 90(13): 5944-8.

Frommer, W. B., S. Hummel, M. Unseld and O. Ninnemann (1995). "Seed and vascular expression of a high-affinity transporter for cationic amino acids in Arabidopsis." Proc Natl Acad Sci U S A 92(26): 12036-40.

Gälweiler, L., C. Guan, A. M ller, E. Wisman, K. Mendgen, A. Yephremov and K. Palme (1998). "Regulation of polar auxin transport by AtPIN1 in Arabidopsis vascular tissue [see comments]." Science 282(5397): 2226-30.

Geldner, N., J. Friml, Y. D. Stierhof, G. Jurgens and K. Palme (2001). "Auxin transport inhibitors block PIN1 cycling and vesicle trafficking." Nature 413(6854): 425-8.

Gersani, M. and T. Sachs (1984). "Polarity reorientation in beans expressed by vascular differentiation and polar auxin transport." Differentiation 25: 205-208.

Gil, P., E. Dewey, J. Friml, Y. Zhao, K. C. Snowden, J. Putterill, K. Palme, M. Estelle and J. Chory (2001). "BIG: a calossin-like protein required for polar auxin transport in Arabidopsis." Genes Dev 15(15): 1985-97. 


\section{Literature}

Giros, B., S. el Mestikawy, N. Godinot, K. Zheng, H. Han, T. Yang-Feng and M. G. Caron (1992). "Cloning, pharmacological characterization, and chromosome assignment of the human dopamine transporter." Mol Pharmacol 42(3): 38390.

Goldberg, R. B., S. J. Barker and L. Perez-Grau (1989). "Regulation of gene expression during plant embryogenesis." Cell 56(2): 149-60.

Goldberg, R. B., G. de Paiva and R. Yadegari (1994). "Plant Embryogenesis: Zygote to Seed." Science 266: 605-614.

Guilfoyle, T., G. Hagen, T. Ulmasov and J. Murfett (1998). "How does auxin turn on genes?" Plant Physiol 118(2): 341-7.

Guilfoyle, T., T. Ulmasov and G. Hagen (1998). "The ARF family of transcription factors and their role in plant hormone responsive transcription." Cell Mol. Life Sci. 54: 619-627.

Hadfi, K., V. Speth and G. Neuhaus (1998). "Auxin-induced developmental patterns in Brassica juncea embryos." Development 125(5): 879-87.

Hamann, T., U. Mayer and G. Jürgens (1999). "The auxin-insensitive bodenlos mutation affects primary root formation and apical-basal patterning in the Arabidopsis embryo." Development 126: 1387-1395.

Harada, J. J. (1999). "Signaling in plant embryogenesis." Curr Opin Plant Biol 2(1): 237.

Hardtke, C. S. and T. Berleth (1998). "The Arabidopsis gene MONOPTEROS encodes a transcription factor mediating embryo axis formation and vascular development." Embo J 17(5): 1405-11.

Harlow, E. and D. Lane (1988). Antibodies: A laboratory manual. Cold Spring Harbor, New York, Cold Spring Harbor Laboratory Press.

Hartshorne, D. J. (1998). "Myosin phosphatase: subunits and interactions." Acta Physiol Scand 164(4): 483-93.

Heckel, T., K. Werner, W. F. Sheridan, C. Dumas and P. M. Rogowsky (1999). "Novel phenotypes and developmental arrest in early embryo specific mutants of maize." Planta 210(1): 1-8.

Higgins, D. G. and P. M. Sharp (1989). "Fast and sensitive multiple sequence alignments on a microcomputer." Comput Appl Biosci 5(2): 151-3.

Hirner, B., W. N. Fischer, D. Rentsch, M. Kwart and W. B. Frommer (1998). "Developmental control of $\mathrm{H}+/$ amino acid permease gene expression during seed development of Arabidopsis." Plant J 14(5): 535-44.

Hudson, A. (2000). "Development of Symmetry in Plants." Annu Rev Plant Physiol Plant Mol Biol 51: 349-370. 
Ingram, G. C., C. Boisnard-Lorig, C. Dumas and P. M. Rogowsky (2000). "Expression patterns of genes encoding HD-ZipIV homeodomain proteins define specific domains in maize embryos and meristems." Plant Journal 22(5): 401-414.

Ingram, G. C., J.-L. Magnard, P. Vergne, C. Dumas and P. M. Rogowsky (1999). "ZmOCL1, an HDGL2 family homeobox gene,, is expressed in the outer cell layer throughout maize development." Plant Mol Biol 40: 343-354.

Johansen, D. A. (1950). Plant Embryology.

Jones, A. M. (1990). "Location of transported auxin in etiolated maize shoots using 5azidoindole-3-acetic acid." Plant Physiol 93: 1154-1161.

Jones, A. M. (1998). "Auxin transport: down and out and up again [comment]." Science 282(5397): 2201-3.

Jürgens, G. (1995). "Axis formation in plant embryogenesis: cues and clues." Cell 81(4): 467-70.

Jürgens, G. (2001). "Apical-basal pattern formation in Arabidopsis embryogenesis." Embo J 20(14): 3609-16.

Jürgens, G., U. Mayer, R. A. Torres Ruiz, T. Berleth and S. Misera (1991). "Genetic analysis of pattern formation in the Arabidopsis embryo." Development (supplement) 1: 27-38.

Jürgens, G., R. A. Torres Ruiz and T. Berleth (1994). "Embryonic pattern formation in flowering plants." Annu Rev Genet 28: 351-71.

Kim do, K., Y. Kanai, H. Matsuo, J. Y. Kim, A. Chairoungdua, Y. Kobayashi, A. Enomoto, S. H. Cha, T. Goya and H. Endou (2002). "The human T-type amino Acid transporter-1: characterization, gene organization, and chromosomal location." Genomics 79(1): 95-103.

Kim, Y. S., D. Kim and J. Jung (1998). "Isolation of a novel auxin receptor from soluble fractions of rice (Oryza sativa L.) shoots." FEBS Lett 438(3): 241-4.

Kishimoto, A., K. Nishiyama, H. Nakanishi, Y. Uratsuji, H. Nomura, Y. Takeyama and Y. Nishizuka (1985). "Studies on the phosphorylation of myelin basic protein by protein kinase $C$ and adenosine 3 ':5'-monophosphate-dependent protein kinase." J Biol Chem 260(23): 12492-9.

Laux, T. and G. Jürgens (1997). "Embryogenesis: A new Start in Life." The Plant Cell 9: 989-1000.

Lazarus, C. M., R. M. Napier, L. X. Yu, C. Lynas and M. A. Venis (1991). "Auxinbinding protein--antibodies and genes." Symp Soc Exp Biol 45: 129-48.

Li, H. M., L. Altschmied and J. Chory (1994). "Arabidopsis mutants define downstream branches in the phototransduction pathway." Genes Dev 8: 339349 . 


\section{Literature}

Li, Y., Z.-B. Liu, X. Shi, G. Hagen and T. Guilfoyle (1994). "Auxin inducible elements in the soybean SAUR promoters." Plant Physiol 106: 37-43.

Liang, P. and A. B. Pardee (1992). "Differential display of eukaryotic messenger RNA by means of the polymerase chain reaction." Science 257: 967-970.

Liu, C., Z. Xu and N. Chua (1993). "Auxin Polar Transport Is Essential for the Establishment of Bilateral Symmetry during Early Plant Embryogenesis." The Plant Cell 5: 621-630.

Liu, Z.-B., T. Ulmasov, X. Shi, G. Hagen and T. Guilfoyle (1994). "The soybean GH3 promoter contains multiple auxin-inducible elements." Plant Cell 6: 645-657.

Lloyd, C. W. (1991). "How does the cytoskeleton read the laws of geometry in aligning the division plane of plant cells?" Development (supplement) 1: 55.

Lomax, T., G. Muday and P. Rubery (1995). Plant Hormones: Physiology, Biochemistry, and Molecular Biology. Boston, Kluwer Academic.

Lopes, M. A. and B. A. Larkins (1993). "Endosperm origin, development and function." The Plant Cell 5: 1383-1399.

Luschnig, C. (2001). "Auxin transport: why plants like to think BIG." Curr Biol 11(20): R831-3.

Luschnig, C., R. A. Gaxiola, P. Grisafi and G. R. Fink (1998). "EIR1, a root-specific protein involved in auxin transport, is required for gravitropism in Arabidopsis thaliana." Genes Dev 12(14): 2175-87.

Mansfield, S. G. and L. G. Briarty (1990). "Early embryogenesis in Arabidopsis thaliana. II. The developing embryo." Can. J. Bot. 69: 461-476.

Marchant, A., J. Kargul, S. T. May, P. Muller, A. Delbarre, C. Perrot-Rechenmann and M. J. Bennett (1999). "AUX1 regulates root gravitropism in Arabidopsis by facilitating auxin uptake within root apical tissues." Embo J 18(8): 2066-2073.

Marger, M. D. and M. H. Saier, Jr. (1993). "A major superfamily of transmembrane facilitators that catalyse uniport, symport and antiport." Trends Biochem Sci 18(1): 13-20.

Mathysse, A. G. and T. K. Scott (1984). Functions of hormones at the whole plant level of organization. Hormonal Regulation of Development II: The Functions of Hormones from the Level of the Cell to the Whole Plant. E. o. P. Physiology. New York, Springer Verlag. 10: 219-243.

Mattsson, J., Z. R. Sung and T. Berleth (1999). "Responses of plant vascular systems to auxin transport inhibition." Development 126(13): 2979-91.

Mayer, U., G. Büttner and G. Jürgens (1993). "Apical-basal pattern formation in the Arabidopsis embryo: studies on the role of the GNOM gene." Development 117: 149-162. 
Mayer, U. and G. Jürgens (1998). "Pattern formation in plant embryogenesis: a reassessment." Semin. Cell Dev. Biol. 9: 187-193.

Mayer, U., R. A. Torres Ruiz, T. Berleth, S. Misera and G. Jürgens (1991). "Mutations affecting body organization in the Arabidopsis embryo." Nature 353: 402-407.

Meinke, D. W. (1991). "Perspectives on genetic analysis of plant embryogenesis." Plant Cell 3: 857-866.

Meinke, D. W. (1995). "Molecular Genetics of Plant Embryogenesis." Annu. Rev. Plant Physiol. Plant Mol. Biol. 46: 369-394.

Meyerowitz, E. M. (1997). "Genetic control of cell division patterns in developing plants." Cell 88(3): 299-308.

Michalek, W., G. Kunzel and A. Graner (1999). "Sequence analysis and gene identification in a set of mapped RFLP markers in barley (Hordeum vulgare)." Genome 42(5): 849-53.

Morris, D. (2000). "Transmembrane auxin carrier systems - dynamic regulators of polar auxin transport." Plant Growth Regul. 32: 161-172.

Morris, D. A. and J. S. Robinson (1998). "Targeting of auxin carriers to the plasma membrane: differential effects of brefeldin A on the traffic of auxin uptake and efflux carriers." Planta 205: 606-612.

Muday, G. K. (2000). "Maintenance of asymmetric cellular localization of an auxin transport protein through interaction with the actin cytoskeleton." Plant Growth Regul. 19: 385-396.

Muday, G. K. and A. DeLong (2001). "Polar auxin transport: controlling where and how much." Trends in Plant Science 6(11): 535-542.

Müller, A., C. Guan, L. Galweiler, P. Tanzler, P. Huijser, A. Marchant, G. Parry, M. Bennett, E. Wisman and K. Palme (1998). "AtPIN2 defines a locus of arabidopsis for root gravitropism control [In Process Citation]." Embo J 17(23): 6903-11.

Napier, R. M. and M. A. Venis (1995). "Auxin action and auxin-binding proteins." New Phytol. 129: 841-850.

Newbigin, E., D. R. Smyth and A. E. Clarke (1995). "Understanding and controlling plant development." TIB 13: 338-343.

Normanly, J. and B. Bartel (1999). "Redundancy as a way of life - IAA metabolism." Curr Opin Plant Biol 2(3): 207-13.

Oka, M., K. Miyamoto, K. Okada and J. Ueda (1999). "Auxin polar transport and flower formation in Arabidopsis thaliana transformed with indoleacetamide hydrolase (iaaH) gene." Plant Cell Physiol 40(2): 231-7. 


\section{Literature}

Okada, K., J. Ueda, M. K. Komaki, C. J. Bell and Y. Shimura (1991). "Requirement of the auxin polar transport system in early stages of Arabidopsis floral bud formation." Plant Cell 3: 677-684.

Palme, K. and L. Galweiler (1999). "PIN-pointing the molecular basis of auxin transport." Curr Opin Plant Biol 2(5): 375-81.

Przemeck, G. K. H., J. Mattson, C. S. Hardtke, R. Sung and T. Berleth (1996). "Studies on the role of the Arabidopsis gene MONOPTEROS in vascular development and plant cell axialization." Planta 200: 229-237.

Raghavan, V. (1976). Experimental embryogenesis in vascular plants. London, Academic Press.

Randolph, L. F. (1936). "Developmental morphology of the caryopsis in maize." Journal of Agricultural Research 53: 881-916.

Rashotte, A. M., S. R. Brady, R. C. Reed, S. J. Ante and G. K. Muday (2000). "Basipetal auxin transport is required for gravitropism in roots of Arabidopsis." Plant Physiol 122(2): 481-90.

Raven, J. A. (1975). "Transport of indole-3-acetic acid in plant cells in relation to $\mathrm{pH}$ and electrical potential gradients, and its significance for polar IAA transport." New Phytol. 74: 163-172.

Reed, R. C., S. R. Brady and G. K. Muday (1998). "Inhibition of auxin movement from the shoot into the root inhibits lateral root development in Arabidopsis." Plant Physiol 118(4): 1369-78.

Reichel, C., J. Mathur, P. Eckes, K. Langenkemper, C. Koncz, J. Schell, B. Reiss and C. Maas (1996). "Enhanced green fluorescence by the expression of an Aequorea victoria green fluorescent protein mutant in mono- and dicotyledonous plant cells." Proc Natl Acad Sci U S A 93(12): 5888-93.

Reinhardt, D., T. Mandel and C. Kuhlemeier (2000). "Auxin regulates the initiation and radial position of plant lateral organs." Plant Cell 12(4): 507-18.

Reizer, J., A. Reizer and M. H. Saier, Jr. (1994). "A functional superfamily of sodium/solute symporters." Biochim Biophys Acta 1197(2): 133-66.

Rubery, P. and A. R. Sheldrake (1974). "Carrier-mediated auxin transport." Planta 188: 101-121.

Ruegger, M., E. Dewey, L. Hobbie, D. Brown, P. Bernasconi, J. Turner, G. K. Muday and M. Estelle (1997). "Reduced naphthylphthalamic acid binding in the tir3 mutant of Arabidopsis is associated with a reduction in polar auxin transport and diverse morphological defects." Plant Cell 9: 745-757. 
Sabatini, S., D. Beis, H. Wolkenfelt, J. Murfett, T. Guilfoyle, J. Malamy, P. Benfey, O. Leyser, N. Bechtold, P. Weisbeek and B. Scheres (1999). "An auxin-dependent distal organizer of pattern and polarity in the Arabidopsis root." Cell 99(5): $463-72$.

Sambrook, J., E. F. Fritsch and T. Maniatis (1989). Molecular cloning: a laboratory manual. Cold Spring Harbor, New York.

Schultz, J., R. R. Copley, T. Doerks, C. P. Ponting and P. Bork (2000). "SMART: a webbased tool for the study of genetically mobile domains." Nucleic Acids Res 28(1): 231-4.

Schultz, J., F. Milpetz, P. Bork and C. P. Ponting (1998). "SMART, a simple modular architecture research tool: identification of signaling domains." Proc Natl Acad Sci U S A 95(11): 5857-64.

Sheridan, W. F. (1988). "Maize developmental genetics: genes of morphogenesis." Annu. Rev. Genet. 22: 353-385.

Sheridan, W. F. and J. K. Clark (1987). "Maize embryogeny: a promising experimental system." Trends Genet. 3: 3-6.

Sheridan, W. F. and J. K. Clark (1993). "Mutational analysis of morphogenesis of the maize embryo." The Plant Journal 3(2): 347-358.

Sheridan, W. F. and M. G. Neuffer (1982). "Maize developmental mutants. Embryos unable to leaf primordia." J. Hered. 73: 318-329.

Sieburth, L. E. (1999). "Auxin is required for leaf vein pattern in Arabidopsis." Plant Physiol 121(4): 1179-90.

Singer, S. J. (1990). "The structure and insertion of integral proteins in membranes." Annual Review of Cell Biology 6: 247-296.

Souter, M. and K. Lindsey (2000). "Polarity and signaling in plant embryogenesis." J Exp Bot 51(347): 971-83.

Steinmann, T., N. Geldner, M. Grebe, S. Mangold, C. L. Jackson, S. Paris, L. Galweiler, K. Palme and G. Jurgens (1999). "Coordinated polar localization of auxin efflux carrier PIN1 by GNOM ARF GEF." Science 286(5438): 316-8.

Stiefel, V., E. L. Becerra, R. Roca, M. Bastida, T. Jahrmann, E. Graziano and P. Puigdomenech (1999). "TM20, a gene coding for a new class of transmembrane proteins expressed in the meristematic tissues of maize." J Biol Chem 274(39): 27734-9.

Stranathan, M., C. Hastings, H. Trinh and J. L. Zimmerman (1989). "Molecular evolution of two actin genes from carrot." Plant Mol Biol 13(4): 375-83.

Sussmann, M. R. and G. Gardner (1980). "Solubilization of the receptor for the N-1naphthylphthalamic acid." Plant Physiol 66: 1074-1078. 


\section{Literature}

Swarup, R., J. Friml, A. Marchant, K. Ljung, G. Sandberg, K. Palme and M. Bennett (2001). "Localization of the auxin permease AUX1 suggests two functionally distinct hormone transport pathways operate in the Arabidopsis root apex." Genes Dev 15(20): 2648-53.

Swarup, R., A. Marchant and M. J. Bennett (2000). "Auxin transport: providing a sense of direction during plant development." Biochem Soc Trans 28(4): 481-5.

Topping, J. F. and K. Lindsey (1997). "Promoter trap markers differentiate structural and positional components of polar development in Arabidopsis." The Plant Cell 9: 1713-1725.

Topping, J. F., V. J. May, P. R. Muskett and K. Lindsey (1997). "Mutations in the HYDRA1 gene of Arabidopsis perturb cell shape and disrupt embryonic and seedling morphogenesis." Development 124: 4415-4424.

Torres Ruiz, R. A. and G. Jürgens (1994). "Mutations in the FASS gene uncouple pattern formation and morphogenesis in Arabidopsis development." Development 120: 2967-2978.

Torres Ruiz, R. A., A. Lohner and G. Jürgens (1996). "The GURKE gene is required for normal organization of the apical region of the Arabidopsis embryo." The Plant Journal 10: 1005-1016.

Ueda, J., M. K. Komaki, K. Okada and Y. Shimura (1991). "Identification and quantitative distribution of indole-3-acetic acid in Brassica junceae Czern." J Plant Physiol 137: 628-630.

Uggla, C., T. Moritz, G. Sandberg and B. Sundberg (1996). "Auxin as a positional signal in pattern formation in plants." Proc Natl Acad Sci U S A 93: 9282-9286.

Ulmasov, T., G. Hagen and T. J. Guilfoyle (1997). "ARF1, a transcription factor that binds to auxin response elements." Science 276: 1865-1868.

Ulmasov, T., Z.-B. Liu, G. Hagen and T. Guilfoyle (1995). "Composite structure of auxin response elements." Plant Cell 7: 1611-1623.

Usdin, T. B., E. Mezey, C. Chen, M. J. Brownstein and B. J. Hoffman (1991). "Cloning of the cocaine-sensitive bovine dopamine transporter." Proc Natl Acad Sci U S A 88(24): 11168-71.

Utsuno, K., T. Shikanai, Y. Yamada and T. Hashimoto (1998). "Agr, an Agravitropic locus of Arabidopsis thaliana, encodes a novel membrane-protein family member." Plant Cell Physiol 39(10): 1111-8.

van Lammeren, A. A. M. (1986). "Developmental morphology and cytology of the young maize embryo (Zea mays L.)." Acta Bot. Neerl. 35: 169-188.

Villalba, J., M. Lützelschwab and R. Serrano (1991). "Immunocytolocalization of plasma-membrane $\mathrm{H}^{+}$-ATPase in maize coleoptiles and enclosed leaves." Planta(185): 458-461. 
Vinogradov, A. E. (2001). "Mirrored genome size distributions in monocot and dicot plants." Acta Biotheor 49(1): 43-51.

Vroemen, C. W., S. Langeveld, U. Mayer, G. Ripper, G. Jürgens, A. Van Kammen and S. C. De Vries (1996). "Pattern formation in the Arabidopsis embryo revealed by position-specific lipid transfer protein gene expression." Plant Cell 8: 783-791.

Wardlaw, C. W. (1955). Embryogenesis in Plants. London.

Weigel, D. (1993). "Patterning the Arabidopsis embryo." Current Biology 3(7): 443-445.

Weigel, D., J. H. Ahn, M. A. Blazquez, J. O. Borevitz, S. K. Christensen, C. Fankhauser, C. Ferrandiz, I. Kardailsky, E. J. Malancharuvil, M. M. Neff, J. T. Nguyen, S. Sato, Z. Y. Wang, Y. Xia, R. A. Dixon, M. J. Harrison, C. J. Lamb, M. F. Yanofsky and J. Chory (2000). "Activation tagging in Arabidopsis." Plant Physiol 122(4): 1003-13.

West, M. A. L. and J. J. Harada (1993). "Embryogenesis in Higher Plants: An Overview." Plant Cell 5: 1361-1369.

Woodgett, J. R., K. L. Gould and T. Hunter (1986). "Substrate specificity of protein kinase C. Use of synthetic peptides corresponding to physiological sites as probes for substrate recognition requirements." Eur J Biochem 161(1): 177-84.

Xu, N., G. Hagen and T. Guilfoyle (1997). "Multiple auxin response modules in the soybean SAUR 15A promoter." Plant Sci. 126: 193-201.

Yeung, E. C. (1980). "Embryogeny of Phaseolus : The role of the suspensor." Z. Pflanzenphysiol. 96: 17-28.

Yeung, E. C. and D. W. Meinke (1993). "Embryogenesis in angiosperms: Development of the suspensor." Plant Cell 5: 1371-1381. 
Acknowledgement 


\section{Acknowledgements}

I would like to thank the following for people for their support during the course of this study.

Prof.Dr. Pere Puigdomènech for supervision of this thesis and for giving me the possibility to work in his laboratory. His office door was always open; you enter with a problem, you leave with a solution.

Dr. Virginia Stiefel for taking my hand during the first steps in molecular biology and to show me much more than techniques of experimental and research work.

Dr. Dolors Ludevid for her advice, support, discussion and that her door always was open for any kind of big or small problem.

Prof.Dr. Dietrich Gradmann and Prof.Dr. Christiane Gatz for acting as Referent and Korreferent, respectively and for their interest in this work.

the crew of the green lab for the brilliant atmosphere: Miriam for her cheerful spirit, for sharing experiences in the lab, the Tetris competitions and the friendship. Blanca for her fight in the same battlefield of protein study and for her "juajuajua". Titi for being the other "guiri" in the lab and for being a good friend. Adolfo, GT, for never losing his staggering direction. Pep for his advice, discussions and his way he sees science and life in general. Ignacio for being the vertebral column of the green lab and his unbreakable faith in Bill Gates. Ramon for never loosing the interest in dekB and the elephants, even from the foggy streets of London. Néstor for his happy and natural character and the donkey races at 7:00 am in Huesca.

the crew of the red lab: Carmen Romera for her spirit of organisation and to know where L-52 is right now. Marga for the discussion, support and for sharing the passion for Menorca. Pau for being a good friend and for seeking together a PBF in the embryos. Lotere for her look to the sky and her smile. Maite for providing fuel and oil to the motor of the department. Mina for being the soul and good spirit in the floors of Genética molecular. 
Amparo for her support and for showing me that genomics can be fun.

Pilar Fontanet for finding always a free space in the greenhouse for my maize plants. Anna Pons and Merce Miquel for converting little drops of transparent liquids in endless rows of ATGC.

Montse, Fathy and Carles for their friendship and the whole department including the people not mentioned especially.

Marta and Emma for the possibility of my first surgical operation, even if it were only a toad, and their great collaboration. Prof.Dr. Manel Palacín for his interest in plant transporters and the discussions.

Shirley Burgues for polishing the english expressions.

Arnt Suckow for his inestimable friendship and for never saying no to all the stuff I asked him to do.

My parents for their infinite support and their faith in me.

Mónica for the last 8 years, the future, Kaira and so much things that can not be all written here.

This work has been carried out with a grant of the European Project BIO4-CT96-0210 and within the framework of the Maizemb Program. 


\section{Biography}

Name: $\quad$ Torben Jahrmann

Date of Birth: $\quad$ 25. October 1971

Place of Birth: Göttingen, Germany

Nationality: German

1977-1981

Primary school Brüder-Grimm-Schule, Göttingen, Germany

1981-1983 Intermediate school Orientierungsstufe Weende-Nord, Göttingen, Germany

1983-1990 High school at the Hainberg-Gymnasium, Göttingen, Germany

1990-1996 Graduate in Biology - Georg-August-Universität in Göttingen, Germany

1993-1994 Academic year with the student exchange programme Erasmus in Barcelona, Spain (Facultad de Biología, Universidad de Barcelona)

$1996-97$

Graduation project in the department Pflanzenphysiologische Fakultät, Georg-August-Universität, Göttingen, Germany.

"Single-channel activity in elongating hypocotyl cells of the soybean root"

Supervisors: PD Dr. G. Thiel and Prof. Dr. D. Gradmann

January 1998 -

April 2002 Ph D at the Georg-August-Universität, Göttingen, Germany Experimental work was done in the Departamento de Genética Molecular of the Instituto de Biología Molecular de Barcelona (IBMB) CID-CSIC, Spain.

Thesis title: "Functional studies on the transmembrane protein encoded by the TM20 gene in maize"

Referent: $\quad$ Prof. Dr. D.Gradmann

Korreferent: $\quad$ Prof. Dr. C. Gatz

Supervisors Spain: $\quad$ Prof. Dr. P.Puigdomènech

Dr. D.Ludevid 